\title{
Models of the muonium to antimuonium transition
}

\author{
Takeshi Fukuyama $\odot,{ }^{1}$ Yukihiro Mimura $\odot,{ }^{2}$ and Yuichi Uesaka $\oplus^{3}$ \\ ${ }^{1}$ Research Center for Nuclear Physics (RCNP), Osaka University, Ibaraki, Osaka 567-0047, Japan \\ ${ }^{2}$ Department of Physical Sciences, College of Science and Engineering, Ritsumeikan University, \\ Shiga 525-8577, Japan \\ ${ }^{3}$ Faculty of Science and Engineering, Kyushu Sangyo University, 2-3-1 Matsukadai, \\ Higashi-ku, Fukuoka 813-8503, Japan
}

(Received 27 August 2021; accepted 22 November 2021; published 24 January 2022)

\begin{abstract}
Muonium is a bound state composed of an antimuon and an electron, and it constitutes a hydrogenlike atom. Because of the absence of the hadronic matter in the bound state, the muonium is a useful probe to explore new physics being free from the hadronic uncertainties. The process of the muonium-toantimuonium transition is considered to be effective to identify fundamental interactions which relate to the lepton flavor and lepton number violation. New experiments are being planned at J-PARC in Japan and CSNS in China, and it is expected to attract more attention in the near future. In this paper, we will study what kind of model can be verified in the next generation of the muonium-to-antimuonium transition search experiments while escaping the limitations from other experiments. Though the transition probability is strongly suppressed by the lepton flavor conservation in the standard model, it can be much larger by the exchanges of neutral and doubly charged bosons, and by box loop diagrams in new physics beyond the standard model. We study the neutrino models with heavy Majorana neutrinos at TeV scale, a type-II seesaw model, left-right models, and models for radiative neutrino masses such as the Zee-Babu model in particular, in addition to other possible models to induce the sizable transition probability, which can be tested in the forthcoming experiments.
\end{abstract}

DOI: 10.1103/PhysRevD.105.015026

\section{INTRODUCTION}

The muonium ( $\left.\mathrm{Mu}: \mu^{+} e^{-}\right)$to antimuonium $\left(\overline{\mathrm{Mu}}: \mu^{-} e^{+}\right)$ transition is an interesting phenomenological possibility [1-4]. There has been no new experimental result of the Mu-to- $\overline{\mathrm{Mu}}$ transition since the Paul Scherrer Institute (PSI) experiment in the late 1990s [5]. The coefficient of the fourfermion transition operator is bounded to be less than $3 \times 10^{-3}$ in the unit of the Fermi constant by the experiment. A brand new idea of the transition experiment is now planning at Japan Proton Accelerator Research Complex (J-PARC) [6]. A new experiment is also planning at China Spallation Neutron Source (CSNS) [7]. It is expected that the bound will be updated for more than one digit by highintensity $\mu^{+}$beam lines. From the theoretical point of view, the Mu-to- $\overline{\mathrm{Mu}}$ transition is an important ingredient to accumulate our knowledge on lepton flavor violation and lepton number violation, and to extract the fundamental interactions in the lepton sector. For these twenty years, there

Published by the American Physical Society under the terms of the Creative Commons Attribution 4.0 International license. Further distribution of this work must maintain attribution to the author(s) and the published article's title, journal citation, and DOI. Funded by SCOAP ${ }^{3}$. are lots of new experimental results: measurements of the parameters in neutrino oscillations, updated results to bound the Mu-to- $\overline{\mathrm{Mu}}$ transition indirectly, and direct bounds on the new particles at the Large Hadron Collider (LHC). We believe that it is worth organizing the models of the $\mathrm{Mu}$-to- $\overline{\mathrm{Mu}}$ transition.

The Mu-to- $\overline{\mathrm{Mu}}$ transition resembles $K^{0}-\overline{K^{0}}$ mixings in the quark sector. The box diagrams via $W$ boson exchanges generate $K^{0}-\overline{K^{0}}$ mixings in the standard model (SM). It is well known that the $K^{0}-\overline{K^{0}}$ mixings are suppressed due to the unitarity of the quark mixing matrix in the SM, but they do not vanish completely because the up-type quark masses are hierarchical, $m_{u} \ll m_{c} \ll m_{t} \sim M_{W}$. Similarly to the $K^{0}-\overline{K^{0}}$ mixings, the Mu-to- $\overline{\mathrm{Mu}}$ transition operator can be potentially generated by the box diagram via $W$ boson exchanges in the SM. However, the transition operator is strongly suppressed $\sim 10^{-30}$ due to the unitarity of the neutrino mixing matrix and tiny neutrino masses, which is very different from the quark sector. If there is a new particle around a $\mathrm{TeV}$ scale and it couples to electrons and muons, the new interaction can induce the Mu-to- $\overline{M u}$ transition in an observable size.

The induced size of the Mu-to- $\overline{\mathrm{Mu}}$ transition via the $\mathrm{TeV}$ scale particles in the models beyond the SM is indirectly restricted by the nonobservation of lepton flavor violating 
(LFV) processes [8,9], such as $\mu \rightarrow e \gamma$ and $\mu^{+} \rightarrow e^{-} e^{+} e^{+}$ $(\mu \rightarrow 3 e)$. We remark that the severely constrained LFV decays are $\Delta L_{e}=-\Delta L_{\mu}= \pm 1$ processes, while the change of the lepton flavor numbers in the Mu-to- $\overline{\mathrm{Mu}}$ transition is $\Delta L_{e}=-\Delta L_{\mu}=-2$. Therefore, if the lepton flavor numbers that new particles carry are odd, one needs the multiple flavor violating interactions to induce the transition operator, and therefore, it turns out that the size of the Mu-to- $\overline{\mathrm{Mu}}$ transition will be much less than the one which can be observed in near-future experiments. However, if the lepton flavor numbers of the new particles are even, the severe experimental constraints can be avoided and an observable size of the Mu-to- $\overline{\mathrm{Mu}}$ transition at near-future experiments can be induced at the tree level.

Those circumstances of the new physics contributions from flavor violation are similar to the meson mixings in the models beyond the SM. In the case of the Mu-to- $\overline{\mathrm{Mu}}$ transition, an additional contribution from the lepton number violation can be considered. The transition operators can be generated by box diagrams in which the mass terms of the fields in the internal lines violate the lepton numbers, even if the interactions conserve the lepton flavor numbers. In this case, the size of the Mu-to- $\overline{\mathrm{Mu}}$ transition relates to the mechanism to generate the proper size of the tiny active neutrino masses.

The purpose of this paper is to scrutinize the models to induce the transition operators in eager anticipation of the new experiments. In particular, we will study in detail those models with neutrino mass production.

We first review the model-independent issues of the Mu-to- $\overline{\mathrm{Mu}}$ transition and $\mathrm{Mu}$ spectroscopy (Sec. II). We next classify the new particles and interactions that causes the Mu-to- $\overline{\mathrm{Mu}}$ transition in the models beyond the SM, and make introductory remarks on how a sizable transition to be observed in the near-future experiments can be induced avoiding the constraints such as LFV processes, which we have briefly mentioned above (Sec. III). After those traditional reviews, we start up the Mu-to- $\overline{\mathrm{Mu}}$ transitions in the orthodox neutrino models: TeV-scale Majorana neutrinos (Sec. IV), type-II seesaw (Sec. V), and left-right models (Sec. VI). We learn how the LFV processes restrict the transition operators in the respective models. The box loop contribution is restricted by the $\mu \rightarrow e \gamma$, and three-body LFV decays such as $\mu \rightarrow 3 e$ restrict the transition operators generated at the tree level.

We also learn the lepton number violation to induce the Mu-to- $\overline{\mathrm{Mu}}$ transition in the orthodox models, and one finds that the Mu-to-Mu transition induced by the lepton number violation is restricted by the natural realization of the sub$\mathrm{eV}$ neutrino masses. We thus study the models with the radiatively generated neutrino masses (Sec. VII), which will be the main issue of this paper. The radiative neutrino mass models fall into two broad categories: models with and without right-handed neutrinos. In the models with right-handed neutrinos, the Dirac neutrino masses are forbidden by a discrete symmetry and the tree-level neutrino mass is absent. The Mu-to- $\overline{\mathrm{Mu}}$ transitions induced by the lepton number violation in such situations are discussed. The so-called Zee-Babu model [10-13] is one of the representative radiative neutrino models without the right-handed SM singlet fermions. We show that the ZeeBabu model can produce the largest Mu-to- $\overline{\mathrm{Mu}}$ transition among the radiative neutrino mass models, which can be tested in near-future experiments.

We also describe other models of the Mu-to- $\overline{\mathrm{Mu}}$ transitions via the tree-level mediator exchanges, including the ones that have been known for a long time: neutral scalar exchange (Sec. VIII), $R$-parity violating supersymmetry (SUSY) (Sec. IX), dilepton gauge bosons (Sec. X), and flavored neutral gauge bosons (Sec. XI). We will also investigate the radiative neutrino masses as a version of a SUSY model with $R$-parity. Other possible exotics can be considered (Sec. XII): leptoquarks, vectorlike fermions, and axionlike particles.

\section{MODEL INDEPENDENT DESCRIPTION OF Mu-to- $\overline{\mathrm{Mu}}$ TRANSITION}

We review the model-independent issues on the $\mathrm{Mu}-\mathrm{to}-\overline{\mathrm{Mu}}$ transitions. We first describe the quantum mechanics of the Mu-to- $\overline{\mathrm{Mu}}$ transition and the probability of the transition. We next introduce the four-fermion operators of the Mu-to- $\overline{\mathrm{Mu}}$ transition, and we obtain the transition amplitudes. Since the experiments for the Mu-to- $\overline{\mathrm{Mu}}$ transition have been done in a magnetic field, we need to know the magnetic field dependence of the transition probability in order to decode the experimental results. We also comment on the corrections to the groundstate $\mathrm{Mu}$ hyperfine structure from the transition operators.

\section{A. Mu $-\overline{M u}$ mixings}

The Schrödinger equation of the $\mathrm{Mu}-\overline{\mathrm{Mu}}$ system is

$$
i \frac{\partial}{\partial t}\left(\begin{array}{l}
\alpha \\
\beta
\end{array}\right)=\left(\begin{array}{ll}
\mathcal{M}_{11} & \mathcal{M}_{12} \\
\mathcal{M}_{21} & \mathcal{M}_{22}
\end{array}\right)\left(\begin{array}{l}
\alpha \\
\beta
\end{array}\right)
$$

for $|\psi(t)\rangle=\alpha(t)|\mathrm{Mu}\rangle+\beta(t)|\overline{\mathrm{Mu}}\rangle$. The matrix elements can be written as $\mathcal{M}_{i j}=M_{i j}-i \Gamma_{i j} / 2$. The $C P T$ symmetry holds $\mathcal{M}_{11}=\mathcal{M}_{22}$, and $\mathrm{Mu}$ and $\overline{\mathrm{Mu}}$ can mix largely even if the off-diagonal element is tiny. Solving the Schrödinger equation, we obtain the time evolution of the Mu state, which is purely $\mathrm{Mu}$ at $t=0$, as

$$
|\mathrm{Mu}(t)\rangle=f_{+}(t)|\mathrm{Mu}\rangle+(q / p) f_{-}(t)|\overline{\mathrm{Mu}}\rangle,
$$

where 


$$
\begin{gathered}
q / p=\sqrt{\frac{\mathcal{M}_{21}}{\mathcal{M}_{12}}}, \quad f_{ \pm}(t)=\frac{1}{2}\left(e^{-i \lambda_{+} t} \pm e^{-i \lambda_{-} t}\right), \\
\lambda_{ \pm}=M-i \frac{\Gamma}{2} \pm \frac{1}{2}\left(\Delta M-i \frac{\Delta \Gamma}{2}\right) \\
\Delta M-i \frac{\Delta \Gamma}{2}=2 \sqrt{\mathcal{M}_{12} \mathcal{M}_{21}}
\end{gathered}
$$

Here, $M$ and $\Gamma$ are the averages of the masses and widths, respectively, and $\Delta M$ and $\Delta \Gamma$ are the differences of them. The transition probability at a time $t$ can be written as

$$
\begin{aligned}
P(\mathrm{Mu} & \rightarrow \overline{\mathrm{Mu}} ; t) \sim|q / p|^{2}\left|f_{-}(t)\right|^{2}, \\
P(\mathrm{Mu} & \rightarrow \mathrm{Mu} ; t) \sim\left|f_{+}(t)\right|^{2},
\end{aligned}
$$

and one can calculate

$$
\left|f_{ \pm}(t)\right|^{2}=\frac{1}{2} e^{-\Gamma t}\left(\cosh \frac{\Delta \Gamma}{2} t \pm \cos \Delta M t\right) .
$$

If there is $C P$ symmetry or $\left|\Gamma_{12} / M_{12}\right| \ll 1$, one obtains $|q / p|=1$. We take a plausible assumption $\left|\Gamma_{12} / M_{12}\right| \ll 1$ to describe the following, and $\mathcal{M} \equiv \mathcal{M}_{12}=(\Delta M) / 2$.

The time-integrated probability of the Mu-to- $\overline{\mathrm{Mu}}$ transition is obtained by

$P(\mathrm{Mu} \rightarrow \overline{\mathrm{Mu}})=\int_{0}^{\infty} d t \Gamma e^{-\Gamma t} \sin ^{2} \mathcal{M} t=\frac{2 \mathcal{M}^{2}}{4 \mathcal{M}^{2}+\Gamma^{2}}$,

which corresponds to the probability that the decay of the $\mathrm{Mu}$ produced in the laboratory comes from the $\mathrm{Mu}\left(\mu^{+} e^{-}\right) \rightarrow \overline{\mathrm{Mu}}\left(\mu^{-} e^{+}\right) \rightarrow\left(e^{-}+\bar{\nu}_{e}+\nu_{\mu}\right)+e^{+}$mode. For $|\Delta M| \ll \Gamma=1 / \tau$ ( $\tau$ is the Mu lifetime, $2.20 \mu \mathrm{s}$ ), one can write the time-integrated transition propability as

$$
P \simeq 2 \tau^{2} \mathcal{M}^{2} .
$$

\section{B. Operators}

The operators which can induce the Mu-to- $\overline{\mathrm{Mu}}$ transition are [14]

$$
\begin{gathered}
Q_{1}=\left(\bar{\mu} \gamma_{\mu}\left(1-\gamma_{5}\right) e\right)\left(\bar{\mu} \gamma^{\mu}\left(1-\gamma_{5}\right) e\right), \\
Q_{2}=\left(\bar{\mu} \gamma_{\mu}\left(1+\gamma_{5}\right) e\right)\left(\bar{\mu} \gamma^{\mu}\left(1+\gamma_{5}\right) e\right), \\
Q_{3}=\left(\bar{\mu} \gamma_{\mu}\left(1+\gamma_{5}\right) e\right)\left(\bar{\mu} \gamma^{\mu}\left(1-\gamma_{5}\right) e\right), \\
Q_{4}=\left(\bar{\mu}\left(1-\gamma_{5}\right) e\right)\left(\bar{\mu}\left(1-\gamma_{5}\right) e\right), \\
Q_{5}=\left(\bar{\mu}\left(1+\gamma_{5}\right) e\right)\left(\bar{\mu}\left(1+\gamma_{5}\right) e\right) .
\end{gathered}
$$

Any dimension-six four-fermion operators for the $\mathrm{Mu}$-to- $\overline{\mathrm{Mu}}$ transition can be written by a linear combination of the above five by using Fierz identities. For example, one can find

$$
\begin{gathered}
\left(\bar{\mu}\left(1+\gamma_{5}\right) e\right)\left(\bar{\mu}\left(1-\gamma_{5}\right) e\right)=-\frac{1}{2} Q_{3}, \\
(\bar{\mu} e)(\bar{\mu} e)=\frac{1}{4}\left(-Q_{3}+Q_{4}+Q_{5}\right), \\
\left(\bar{\mu} \gamma_{5} e\right)\left(\bar{\mu} \gamma_{5} e\right)=\frac{1}{4}\left(Q_{3}+Q_{4}+Q_{5}\right), \\
\left(\bar{\mu} \sigma_{\mu \nu} e\right)\left(\bar{\mu} \sigma^{\mu \nu} e\right)=-3\left(Q_{4}+Q_{5}\right) .
\end{gathered}
$$

We denote the terms in the effective Lagrangian as

$$
-\mathcal{L}_{\mathrm{Mu}-\overline{\mathrm{Mu}}}=\sum_{i=1, \ldots, 5} \frac{G_{i}}{\sqrt{2}} Q_{i},
$$

where the normalization of the coefficients mimics the Fermi constant $G_{F}$.

In practice, the state of the produced $\mathrm{Mu}$ is a mixture of four states made by the hyperfine structure. The four states $(F, m)=(0,0),(1,0)$, and $(1, \pm 1)$ are indicated by the magnitude of the total angular momentum $F$ and the $z$ component of the total angular momentum $m$. The $F=0$ state is called paramuonium, while the $F=1$ state is called orthomuonium. The amplitudes of the $\operatorname{Mu}(F, m) \rightarrow$ $\overline{\mathrm{Mu}}(F, m)$ transition $^{1}$ are written as

$$
\mathcal{M}_{F, m}=\sum_{i=1, \ldots, 5} \frac{G_{i}}{\sqrt{2}}\left\langle\overline{\mathrm{Mu}} ; F, m\left|Q_{i}\right| \mathrm{Mu} ; F, m\right\rangle .
$$

Treating the bound leptons nonrelativistically, we obtain

$$
\mathcal{M}_{0,0}=-\frac{8|\varphi(0)|^{2}}{\sqrt{2}}\left(G_{1}+G_{2}-\frac{3}{2} G_{3}-\frac{1}{4} G_{4}-\frac{1}{4} G_{5}\right),
$$

for the spin-singlet paramuonium, and

$$
\begin{aligned}
\mathcal{M}_{1,0} & =\mathcal{M}_{1, \pm 1} \\
& =-\frac{8|\varphi(0)|^{2}}{\sqrt{2}}\left(G_{1}+G_{2}+\frac{1}{2} G_{3}-\frac{1}{4} G_{4}-\frac{1}{4} G_{5}\right),
\end{aligned}
$$

for the spin-triplet orthomuonium. The derivation of Eqs. (2.20) and (2.21) is given in Appendix A.

Solving the Schrödinger equation for the hydrogenlike atom, we find that the wave function $\varphi(\boldsymbol{r})$ is

$$
\varphi(\boldsymbol{r})=\sqrt{\frac{\left(m_{\mathrm{red}} \alpha\right)^{3}}{\pi}} \exp \left(-m_{\mathrm{red}} \alpha r\right)
$$

\footnotetext{
${ }^{1}$ If $F \neq F^{\prime}$ or $m \neq m^{\prime},\left\langle\overline{\mathrm{Mu}} ; F, m\left|Q_{i}\right| \mathrm{Mu} ; F^{\prime}, m^{\prime}\right\rangle=0$ for any $i$.
} 
where $m_{\text {red }}=m_{e} m_{\mu} /\left(m_{\mu}+m_{e}\right) \simeq m_{e}$ is the reduced mass between a muon and an electron and $\alpha \simeq 1 / 137$ is the QED fine structure constant. The value of $\varphi(\boldsymbol{r})$ at the origin is given by

$$
|\varphi(0)|^{2}=\frac{\left(m_{\mathrm{red}} \alpha\right)^{3}}{\pi}
$$

\section{Magnetic field dependence}

The transition probability of $\mathrm{Mu}$ to $\overline{\mathrm{Mu}}$ is changed in a finite magnetic field $B$. Since we have to care about the effects of the external magnetic field to describe the experimental constraints given by the PSI experiment, let us review the magnetic field dependence $[15,16]$. For the Mu spectroscopy, see Appendix B.

The external magnetic field splits the $(1, \pm 1)$ states and makes $\Delta E=M_{11}-M_{22}$ to be nonzero. As a consequence, the $\mathrm{Mu}-\overline{\mathrm{Mu}}$ mixing becomes small and the transition probability becomes

$$
\begin{aligned}
P(\mathrm{Mu} \rightarrow \overline{\mathrm{Mu}}, t) \simeq & e^{-\Gamma t} \frac{\mathcal{M}_{1, \pm 1}^{2}}{\mathcal{M}_{1, \pm 1}^{2}+(\Delta E / 2)^{2}} \\
& \times \sin ^{2} \sqrt{\mathcal{M}_{1, \pm 1}^{2}+(\Delta E / 2)^{2}} t,
\end{aligned}
$$

and the time-integrated probability is

$$
\int_{0}^{\infty} d t \Gamma P(\mathrm{Mu} \rightarrow \overline{\mathrm{Mu}}, t) \simeq \frac{2 \tau^{2}\left|\mathcal{M}_{1, \pm 1}\right|^{2}}{1+(\tau \Delta E)^{2}} .
$$

The energy splitting $\Delta E$ of the $(1, \pm 1)$ states by the magnetic field can be obtained by Eq. (B3), and one obtains

$$
\tau \Delta E=3.85 \times 10^{5} \times \frac{B}{\text { Tesla }} .
$$

Therefore, in the magnetic flux $B$ to be more than $1 \mu T$ (micro Tesla), the transition probability is suppressed for $(1, \pm 1)$ states. For one's information, the geomagnetic flux density is $\sim 30-60 \mu T$.

We note that the oscillation time without a magnetic field is $O(1)$ second or longer under the current experimental bound. Therefore, the Mu-to- $\overline{\mathrm{Mu}}$ "oscillations" do not start before $\mathrm{Mu}$ decays. The behavior of the transition probability for the $m= \pm 1$ states near $t=0$ is the same as the one without a magnetic field. However, if the external magnetic field is $\sim 1 \mu T$, the oscillation time is the same as the Mu lifetime, and therefore, the transition probability for $m= \pm 1$ is suppressed for $B \gtrsim O(1) \mu T$.

The $\mathrm{Mu}-\overline{\mathrm{Mu}}$ mixing for $m=0$ states is (nearly) maximal even in the magnetic field, contrary to the $m= \pm 1$ states. The $(F, m)=(1,0)$ and $(0,0)$ states are mixed due to the magnetic filed, and the transition amplitudes (halves of the mass differences) are modified as

$$
\begin{gathered}
\mathcal{M}_{0,0}^{B}=\frac{1}{2}\left(\mathcal{M}_{0,0}-\mathcal{M}_{1,0}+\frac{\mathcal{M}_{0,0}+\mathcal{M}_{1,0}}{\sqrt{1+X^{2}}}\right), \\
\mathcal{M}_{1,0}^{B}=\frac{1}{2}\left(-\mathcal{M}_{0,0}+\mathcal{M}_{1,0}+\frac{\mathcal{M}_{0,0}+\mathcal{M}_{1,0}}{\sqrt{1+X^{2}}}\right),
\end{gathered}
$$

where $X=6.31 \times B /$ Tesla is defined in Eq. (B6).

The time-integrated transition probability is totally

$$
\begin{aligned}
P= & 2 \tau^{2}\left(\left|c_{0,0}\right|^{2}\left|\mathcal{M}_{0,0}^{B}\right|^{2}+\left|c_{1,0}\right|^{2}\left|\mathcal{M}_{1,0}^{B}\right|^{2}\right. \\
& \left.+\sum_{m= \pm 1}\left|c_{1, m}\right|^{2} \frac{\left|M_{1, m}\right|^{2}}{1+(\tau \Delta E)^{2}}\right)
\end{aligned}
$$

where $\left|c_{F, m}\right|^{2}$ gives the population of the Mu states. The experimental result by the PSI experiment at the magnetic flux density $B=0.1$ Tesla is [5]

$$
P<8.3 \times 10^{-11} .
$$

The oscillations of the $(1, \pm 1)$ states are dropped in the magnetic flux density. If $G_{3}=0$, we obtain

$$
\begin{aligned}
P= & \frac{64 m_{\mathrm{red}}^{6} \alpha^{6} \tau^{2} G_{F}^{2}}{\pi^{2}}\left(\frac{G_{1}+G_{2}-\frac{1}{4}\left(G_{4}+G_{5}\right)}{G_{F}}\right)^{2} \\
& \times \frac{\left|c_{0,0}\right|^{2}+\left|c_{1,0}\right|^{2}}{1+X^{2}} \\
= & 2.57 \times 10^{-5}\left(\frac{G_{1}+G_{2}-\frac{1}{4}\left(G_{4}+G_{5}\right)}{G_{F}}\right)^{2} \\
& \times \frac{\left|c_{0,0}\right|^{2}+\left|c_{1,0}\right|^{2}}{1+X^{2}},
\end{aligned}
$$

and the experimental result is decoded as

$$
\left|G_{1}+G_{2}-\frac{1}{4} G_{4}-\frac{1}{4} G_{5}\right|<3.0 \times 10^{-3} G_{F} .
$$

If $G_{3} \neq 0$ and the others are zero, we find

$$
\left|G_{3}\right|<2.1 \times 10^{-3} G_{F} .
$$

We use the population of $\mathrm{Mu}$ states, $\left|c_{0,0}\right|^{2}=0.32$, $\left|c_{1,0}\right|^{2}=0.18$. If the operators are turned on containing $Q_{3}$, the expression is a little complicated to write down here, but one can easily calculate the bound from the expressions above.

The PSI experiment tried to observe the decay product (electron) from an expected $\mu^{-}$in $\overline{\mathrm{Mu}}\left(\mu^{-} e^{+}\right)$after $\mathrm{Mu}$ $\left(\mu^{+} e^{-}\right)$is produced in the laboratory. The external magnetic field is thus needed. The MACE group in China will also use this method [7]. The time is not specified, and the timeintegrated transition probability is applied. The intrinsic beam-related and accidental backgrounds disturb the 
detection of the electrons emitted from the $\overline{M u}$ decays, which determines the experimental bound of the Mu-to- $\overline{M u}$ transition.

A new method of the Mu-to- $\overline{\mathrm{Mu}}$ transition search is proposed using a high-intensity pulsed muon source in J-PARC and an intense laser [6]. The high-intensity beam (H-line) will work this summer. In their method, an expected $\overline{\mathrm{Mu}}$ is ionized by a laser shot at a time and the dissolved $\mu^{-}$is directly analyzed by a spectrometer. The transition probability is not time-integrated and is given at a time $t$ when the laser is shot. Therefore, the number of the possible transition events will be less (by a factor $(t / \tau)^{2} \exp (-t / \tau) / 2$ up to the other experimental lacks in the laboratory) than the time-integrated transitions in the preceding method. This method, however, is free from the background noises from the accelerator and messy positrons' scatterings from $\mu^{+}$decays in the preceding experiment. Their method does not need an external magnetic flux to detect the decay products from $\overline{\mathrm{Mu}}$ and $\mathrm{Mu}$ decays, and the Mu-to- $\overline{M u}$ transition can be observed with the geomagnetic flux or with shielding it. The controllability of the external magnetic field has further advantages to confirm new physics and to investigate the operator dominance by the characteristic magnetic-field dependence.

\section{Muonium hyperfine structure}

The MuSEUM group is planning measurements of the $1 S$ hyperfine structure (HFS) of $\mathrm{Mu}$ using the $\mathrm{H}$-line at J-PARC [17,18]. The current most accurate experimental value of the Mu HFS interval is [19]

$$
\nu_{\mathrm{HFS}}^{\exp }=4463302765 \pm 53 \mathrm{~Hz}
$$

which has been measured in a strong magnetic field. The MuSEUM group will reduce the systematic errors of the measurements to a few $\mathrm{Hz}$ in both zero and strong magnetic fields. The theoretical expression of the HFS interval can be written in Heaviside-Lorentz units as

$\nu_{\mathrm{HFS}}^{\mathrm{th}}=\frac{1}{4 \pi} \frac{16}{3} \mu_{\mu} \mu_{e}|\varphi(0)|^{2}\left(1+\delta_{\mathrm{QED}}+\delta_{\text {weak }}+\delta_{\text {hadronic }}\right)$,

where $\mu_{\mu}$ and $\mu_{e}$ are the magnetic moments of the muon and electron, respectively. The theoretical calculation with electroweak and intermediate hadronic corrections contains the uncertainty $\sim 300-500 \mathrm{~Hz}[20-22]$. Precise measurement by MuSEUM also reduces the uncertainty in the muon-proton magnetic moment and muon-electron mass ratios, which can reduce the uncertainty in the theoretical calculation of the HFS interval. In this subsection, we describe the corrections from the Mu-to- $\overline{\mathrm{Mu}}$ transition operators to the HFS interval. For the ground-state $\mathrm{Mu}$ spectroscopy, see Appendix B.

When the external magnetic field is zero $B=0$, the HFS interval is defined as

$$
h \nu_{\mathrm{HFS}}(B=0) \equiv E_{F=1}-E_{F=0},
$$

where

$E_{F=1}=E_{0}+\frac{1}{4} h \nu_{\mathrm{HFS}}, \quad E_{F=0}=E_{0}-\frac{3}{4} h \nu_{\mathrm{HFS}}$,

and the Planck constant is $h=2 \pi$ since we are working in the natural unit $\hbar=1\left(=6.582 \times 10^{-25} \mathrm{GeV} \cdot \mathrm{s}\right)$. When there is a transition operator, the $\mathrm{Mu}$ and $\overline{\mathrm{Mu}}$ is maximally mixed (even if the coefficient of the operator is small). The energy eigenstates correspond to $C P$ eigenstates. The HFS interval is measured by using the resonance of microwave frequency in the cavity. The transitions to the different $C P$ states are suppressed. As a result, the correction from the transition operators is given as

$$
\begin{aligned}
\Delta \nu_{\mathrm{HFS}}(B=0) & = \pm \frac{M_{F=1}-M_{F=0}}{2 \pi} \\
& = \pm \frac{4 m_{\mathrm{red}}^{3} \alpha^{3}}{\sqrt{2} \pi^{2}} 2\left|G_{3}\right| .
\end{aligned}
$$

If there is $Q_{3}$, the mass difference between $\mathrm{Mu}$ and $\overline{\mathrm{Mu}}$ is different for spin-singlet and triplet, and then, it can modify the Mu HFS interval between the spin-singlet and triplet [23]. If there is only a $Q_{3}$ operator, the current bound of the Mu-to- $\overline{M u}$ transition implies

$$
\left|\Delta \nu_{\mathrm{HFS}}(B=0)\right|<1.1 \mathrm{~Hz} .
$$

When there is an external magnetic field, the states split and thus the definition of the HFS interval should be modified:

$$
\begin{aligned}
h \nu_{\mathrm{HFS}}(B \neq 0) & =h \nu_{12}+h \nu_{34} \\
& \equiv E_{(1,1)}-E_{(1,0)}+E_{(1,-1)}-E_{(0,0)} .
\end{aligned}
$$

The energy eigenstates are not $C P$ eigenstates in the magnetic field, and thus there are two resonant frequencies if there is a transition operator and the measurements of the frequency are very accurate. The corrections are given as

$$
\begin{aligned}
\Delta \nu_{12} & \simeq \pm \frac{M_{1,0}^{B}}{2 \pi} \\
& = \pm \frac{4 m_{\mathrm{red}}^{3} \alpha^{3}}{\sqrt{2} \pi^{2}}\left|G_{3}+\frac{G_{1}+G_{2}-\frac{1}{2} G_{3}-\frac{1}{4} G_{4}-\frac{1}{4} G_{5}}{\sqrt{1+X^{2}}}\right|,
\end{aligned}
$$




$$
\begin{aligned}
\Delta \nu_{34} & \simeq \pm \frac{M_{0,0}^{B}}{2 \pi} \\
& = \pm \frac{4 m_{\mathrm{red}}^{3} \alpha^{3}}{\sqrt{2} \pi^{2}}\left|-G_{3}+\frac{G_{1}+G_{2}-\frac{1}{2} G_{3}-\frac{1}{4} G_{4}-\frac{1}{4} G_{5}}{\sqrt{1+X^{2}}}\right| .
\end{aligned}
$$

The splitting of the resonant frequency is less than about $1 \mathrm{~Hz}$ for the current experimental bound of the Mu-to-Mu transition.

The theoretical calculation of the HFS interval contains the uncertainty of the fine structure constant, and it will be hard to reduce the uncertainty of the theoretical prediction to be less than $1 \mathrm{~Hz}$. Therefore, we cannot say anything about new physics even if the HFS interval is accurately measured only in the case of $B=0$. The accurate measurements of the HFS intervals for both $B=0$ and $B \neq 0$ will be important. According to Ref. [18], the HFS intervals for both $B \simeq 0$ and $B \neq 0$ will be measured with systematic errors of 2-3 Hz. The accurate measurement of the HFS intervals will give us an interesting cross-check, though the accuracy is not enough to say something, and the Mu-to- $\overline{\mathrm{Mu}}$ transition bound will be updated when the HFS interval is accurately measured at J-PARC.

\section{CLASSIFICATION OF THE MEDIATORS}

The purpose of this paper is to study models to induce the transition operators, $Q_{i}$. Before moving to the concrete description of the individual models, we classify them by the LFV couplings to generate the operators to learn how the Mu-to- $\overline{\mathrm{Mu}}$ transition can be sizable avoiding the LFV decay constraints. Though the assignments of the lepton (flavor) numbers may have ambiguity in respective models, this classification can specify the mediator in the model. This classification is useful to make clear what is needed to obtain the sizable Mu-to- $\overline{\mathrm{Mu}}$ transition in a modelindependent way.

(1) $\Delta L_{e}=\Delta L_{\mu}=0$

The interactions do not violate the flavor numbers, but the mass terms of SM singlet fields have $L_{e}= \pm 2$ and $L_{\mu}= \pm 2$. Total lepton number conservation is violated in this case. Therefore, this case is friendly to the models to generate neutrino masses.

The models with right-handed Majorana neutrinos are considered to induce the $\mathrm{Mu}$-to- $\overline{\mathrm{Mu}}$ transition, which will be studied mainly in Secs. IV, VII B, and VIIC. The transition operators can be generated by box loop diagrams [e.g., Figs. 1 (right) and 12 (right)].

(2) $\left(\Delta L_{e}, \Delta L_{\mu}\right)=( \pm 2,0)$ and $\left(\Delta L_{e}, \Delta L_{\mu}\right)=(0, \pm 2)$ Interaction terms violate the flavor numbers separately.
The mediators have the lepton number to be 2, and they are called dilepton bosons. The mediators have doubly electric charges. The dilepton doubly charged scalars will be studied in Secs. V, VI, and VII A, and dilepton gauge boson will be considered in Sec. X. The transition operators can be generated by tree diagrams (e.g., Figs. 3 and 16).

(3) $\Delta L_{e}=-\Delta L_{\mu}= \pm 1$

Interaction terms violate both flavor numbers.

The mediators are neutral bosons. The neutral scalars are considered in Secs. VIII and IX A, and neutral gauge bosons are studied in Sec. XI. The transition operators can be generated by tree diagram (e.g., Figs. 15 and 17).

We remark that this mediator should not couple with quarks to generate the Mu-to- $\overline{\mathrm{Mu}}$ transition to avoid the $\mu-e$ conversion in nuclei induced at the tree level.

(4) $\left(\Delta L_{e}, \Delta L_{\mu}\right)=( \pm 1,0)$ and $\left(\Delta L_{e}, \Delta L_{\mu}\right)=(0, \pm 1)$

The transition operators can be generated by box loop diagrams [e.g., Figs. 1 (left) and 12 (left)]. However, the interaction can induce $\mu \rightarrow e \gamma$ and/or $\mu \rightarrow 3 e$, which restricts the size of coupling constants. Then, the magnitudes of the coefficients of the transition operators become much less than the achievement of the planned experiments, as we will see in many of the models.

In the cases 1,2 , and 3, there are interactions or mass terms with $\Delta L_{e}-\Delta L_{\mu}= \pm 2$, which are even numbers. Even in those cases, the interactions with $\Delta L_{e}-\Delta L_{\mu}=$ \pm 1 may be intermingled in the respective models, and then, the magnitudes of the Mu-to- $\overline{\mathrm{Mu}}$ transition is bounded by $\mu \rightarrow e \gamma$ and/or $\mu \rightarrow 3 e[8,9]$ :

$$
\begin{aligned}
& \operatorname{Br}(\mu \rightarrow e \gamma)<4.2 \times 10^{-13}, \\
& \operatorname{Br}(\mu \rightarrow 3 e)<1.0 \times 10^{-12} .
\end{aligned}
$$

If $Z_{n}$ discrete symmetry can be imposed to suppress the $\Delta L_{e}-\Delta L_{\mu}= \pm 1$ interactions (while the $\Delta L_{e}-\Delta L_{\mu}=$ \pm 2 interactions are allowed), the Mu-to- $\overline{M u}$ transition can be as large as the current experimental bound. In other words, the observation of the Mu-to- $\overline{\mathrm{Mu}}$ transition in the near-future experiments implies the existence of such discrete symmetry in the lepton sector.

The new $\Delta L_{e}-\Delta L_{\mu}= \pm 2$ interactions can induce a "wrong muon decay":

$$
\mu^{+} \rightarrow \nu_{\mu}+e^{+}+\bar{\nu}_{e},
$$

and thus the couplings and masses of the mediators are restricted by the universality of the Fermi decay constant [23]. They are also constrained by $e^{+} e^{-} \rightarrow e^{+} e^{-}$Bhabha scattering data. The couplings can also induce muon and electron anomalous magnetic moments. The couplings and the 

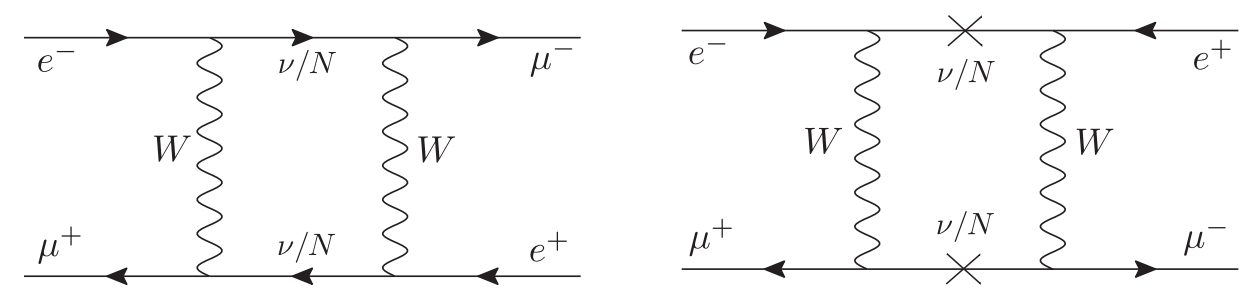

FIG. 1. The box loop diagrams to generate the transition operator $Q_{1}$. There are two types of contribution: the momentum parts ( $\not$ ) of the numerators of neutrino propagators are picked (left), and the mass parts of the propagators are picked (right). The lepton flavors are changed at the vertices in the left diagram, while the lepton numbers are violated in the neutrino masses in the right diagram.

mediator masses to induce the $\mathrm{Mu}-\mathrm{to}-\overline{\mathrm{Mu}}$ transition which is allowed by the PSI experiment, do not conflict with those low energy data at present. Rather, the experimental results of the Mu-to- $\overline{\mathrm{Mu}}$ transitions restrict them. The data from the highluminosity LHC, ILC, and Belle II will cooperate with the near-future $\mathrm{Mu}$-to- $\overline{\mathrm{Mu}}$ transition experiments.

\section{HEAVY MAJORANA NEUTRINOS}

The simplest neutrino model to acquire the Mu-to- $\overline{\mathrm{Mu}}$ transition may be the models with $\mathrm{TeV}$ scale Majorana neutrinos, which are SM singlets. The coefficient of the transition operator from the neutrino box loop contribution is written as [24-27]

$$
\begin{aligned}
\frac{G_{1}}{\sqrt{2}} & =\frac{G_{F}^{2} M_{W}^{2}}{16 \pi^{2}} \sum_{\mathcal{I}, \mathcal{J}}\left[\mathcal{U}_{\mu_{L}} \mathcal{U}_{e_{L} \mathcal{I}}^{*} \mathcal{U}_{\mu_{L}} \mathcal{J}_{e_{L} \mathcal{J}}^{*} E_{0}\left(x_{\mathcal{I}}, x_{\mathcal{J}}\right)+\left(\mathcal{U}_{\mu_{L} \mathcal{I}}\right)^{2}\left(\mathcal{U}_{e_{L} \mathcal{J}}^{*}\right)^{2} E_{1}\left(x_{\mathcal{I}}, x_{\mathcal{J}}\right)\right] \\
& \simeq \frac{G_{F}^{2} M_{W}^{2}}{16 \pi^{2}} \sum_{I, J}\left[X_{\mu I} X_{e I}^{*} X_{\mu J} X_{e J}^{*} \tilde{E}_{0}\left(x_{I}, x_{J}\right)+\left(X_{\mu I}\right)^{2}\left(X_{e J}^{*}\right)^{2} E_{1}\left(x_{I}, x_{J}\right)\right]
\end{aligned}
$$

where

$$
x_{\mathcal{I}}=\frac{M_{\mathcal{I}}^{2}}{M_{W}^{2}}
$$

and see Appendices $\mathrm{C}$ and $\mathrm{D}$ for the neutrino mixing matrix $\mathcal{U}$ and the loop functions $E_{0}$ and $E_{1}$. The $E_{0}$ term comes from box diagrams such as Fig. 1 (left), and $E_{1}$ term comes from diagrams such as Fig. 1 (right). We ignore the light neutrino masses $x_{i}=m_{i}^{2} / M_{W}^{2} \simeq 0$, and one can rewrite the first line into the second line by using the unitary relation of the mixing matrix,

$$
\sum_{\mathcal{I}} \mathcal{U}_{\mu_{L} \mathcal{I}} \mathcal{U}_{e_{L} \mathcal{I}}^{*}=\sum_{i} U_{\mu i} U_{e i}^{*}+\sum_{I} X_{\mu I} X_{e I}^{*}=0
$$

and

$$
\begin{aligned}
\tilde{E}_{0}(x, y) & =E_{0}(x, y)-E_{0}(x, 0)-E_{0}(0, y)+E_{0}(0,0) \\
& =E_{0}(x, y) .
\end{aligned}
$$

We enumerate the necessary facts to evaluate the loop contribution.

(1) The mixings are bounded by electroweak precision data model-independently [28,29]

$$
\left|X_{e I}\right|^{2},\left|X_{\mu I}\right|^{2} \lesssim 0.003
$$

individually (supposing only one of $M_{I}$ is in the $\mathrm{TeV}$ scale).
(2) The product of $\left|X_{e I} X_{\mu I}\right|$ is bounded by LFV processes, especially $\mu \rightarrow e \gamma$.

The $\mu_{R} \rightarrow e_{L} \gamma$ decay amplitude is given as

$$
\begin{aligned}
A_{R} & =\frac{e m_{\mu}}{16 \pi^{2}} \frac{G_{F}}{\sqrt{2}}\left(\sum_{i} U_{\mu i} U_{e i}^{*} F\left(x_{i}\right)+\sum_{I} X_{\mu I} X_{e I}^{*} F\left(x_{I}\right)\right) \\
& =\frac{e m_{\mu}}{16 \pi^{2}} \frac{G_{F}}{\sqrt{2}} \sum_{I} X_{\mu I} X_{e I}^{*} \tilde{F}\left(x_{I}\right),
\end{aligned}
$$

where

$$
\begin{aligned}
\tilde{F}(x) & =F(x)-F(0) \\
& =-\frac{x\left(1-6 x+3 x^{2}+2 x^{3}-6 x^{2} \ln x\right)}{(1-x)^{4}} .
\end{aligned}
$$

One finds ${ }^{2}$

${ }^{2}$ The decay width in our convention of the amplitude is

$$
\Gamma(\mu \rightarrow e \gamma)=\frac{m_{\mu}^{3}}{16 \pi^{2}}\left(\left|A_{L}\right|^{2}+\left|A_{R}\right|^{2}\right) .
$$

The branching ratio can be written as

$$
\operatorname{Br}(\mu \rightarrow e \gamma)=\frac{3 \alpha}{16 \pi}\left(\left|\tilde{A}_{L}\right|^{2}+\left|\tilde{A}_{R}\right|^{2}\right),
$$

where the dimensionless amplitude $\tilde{A}_{L, R}$ is defined by

$$
A_{L, R}=\frac{e m_{\mu}}{16 \pi^{2}} G_{F} \tilde{A}_{L, R}
$$




$$
\operatorname{Br}(\mu \rightarrow e \gamma)=\frac{3 \alpha}{32 \pi}\left|\sum_{I} X_{\mu I} X_{e I}^{*} \tilde{F}\left(x_{I}\right)\right|^{2},
$$

and the bound of $\operatorname{Br}(\mu \rightarrow e \gamma)<4.2 \times 10^{-13}$ requires

$$
\left|\sum_{I} X_{\mu I}^{*} X_{e I} \tilde{F}\left(x_{I}\right)\right| \lesssim 4.4 \times 10^{-5} .
$$

(3) In the box loop contribution of the Mu-to- $\overline{\mathrm{Mu}}$ transition in Eq. (4.1), the $E_{0}$ term is generated by flavor violation, and therefore, its magnitude is bounded by $\mu \rightarrow e \gamma$. The $E_{1}$ term, on the other hand, is generated by the Majorana property of heavy neutrinos even if there is no flavor violation in principle, i.e., the $E_{1}$ term can be enlarged without a constraint from $\mu \rightarrow e \gamma$, if $X_{e I}$ and $X_{\mu J}(I \neq J)$ can become large.

(4) One finds

$$
\begin{aligned}
& E_{0}(x, y) \sim \frac{1}{4} \frac{x y}{x-y} \ln \frac{x}{y}, \\
& E_{1}(x, y) \sim \frac{1}{2} \sqrt{x y} \frac{y \ln x-x \ln y}{x-y},
\end{aligned}
$$

and

$E_{0}(x, x) \sim \frac{1}{4} x, \quad E_{1}(x, x) \sim-\frac{1}{2} x \ln x$,

for large $x, y$. Therefore, "if the neutrino mixings can be kept the same," the Mu-to- $\overline{\mathrm{Mu}}$ transition can be larger for heavier neutrinos. This is due to the longitudinal modes of gauge bosons in the unitary gauge (or Nambu-Goldstone bosons in the 't HooftFeynman gauge).

(5) For one generation $(2 \times 2$ neutrino mass matrix $)$, the light neutrino mass in type-I seesaw is $m_{\nu}=$ $m_{D}^{2} / M_{N}$, and the mixing is $\left(X_{\alpha I}\right)^{2}=m_{D}^{2} / M_{N}^{2}=$ $m_{\nu} / M_{N}$, and therefore, the Mu-to- $\overline{\mathrm{Mu}}$ transition is tiny. For a three-generation case, there is freedom to enlarge the mixings, $X_{e I}$ and $X_{\mu I}$, while keeping the active neutrino masses tiny. Therefore, the $E_{0}$ term can be larger than the naive expectation from the size of the light-heavy neutrino mixing in one generation. If two $X_{\alpha I}$ 's (say $X_{\alpha 1}, X_{\alpha 2}$ ) are large, the heavy neutrino masses need to be degenerate, $M_{1}=-M_{2}$ and $X_{\alpha 1}=X_{\alpha 2}$ (or conventionally, $M_{1}=M_{2}$ and $X_{\alpha 1}=i X_{\alpha 2}$ ), due to the freedom of the mass matrix. Such degeneracy can eliminate the $E_{1}$ contribution. If there are more than three singlet neutrinos, such degeneracy can be released.

(6) If the light-heavy neutrino mixing is enlarged, a sizable active neutrino mass can be generated by $Z$ boson loop [30],
$\left(M_{\nu}\right)_{\alpha \beta} \simeq \frac{\alpha_{2}}{4 \pi \cos ^{2} \theta_{W}} \sum_{I} X_{\alpha I} X_{\beta I} \frac{M_{I}^{3}}{M_{I}^{2}-M_{Z}^{2}} \ln \frac{M_{Z}^{2}}{M_{I}^{2}}$

The loop-induced neutrino mass can be canceled if the heavy neutrino masses are degenerate $\left(M_{1}=-M_{2}\right)$. Therefore, the $E_{1}$ contribution cannot be enlarged, unless the tree-level and loop-induced active neutrino masses are miraculously canceled. (Even if one allows such unnatural cancellation, the size of the coefficient is $\left|G_{1}\right| \lesssim O\left(10^{-6}\right) G_{F}$ due to the constraints of light-heavy neutrino mixings from precision electroweak data, and near-future experiments cannot reach it.)

In total, the $E_{0}$ contribution is bounded by $\mu \rightarrow e \gamma$ constraint, and the $E_{1}$ contribution is bounded by the natural neutrino mass hierarchy.

Since the cancellation between the tree-level and loopinduced neutrino masses cannot be controlled by symmetry, an elaborated construction of the neutrino mass model is needed (e.g., the Dirac neutrino mass is forbidden) to enlarge the $\mathrm{Mu}$-to- $\overline{\mathrm{Mu}}$ transition naturally from the neutrino Majorana property, which we will see in Sec. VII. Here, we exhibit the Mu-to- $\overline{\mathrm{Mu}}$ transition assuming the heavy neutrino mass degeneracy, which can be controlled by a flavor symmetry.

We assume that the right-handed neutrino mass matrix $M_{N}$ is given as

$$
M_{N}=\left(\begin{array}{ccc}
0 & 0 & M_{1} \\
0 & M_{3} & 0 \\
M_{1} & 0 & 0
\end{array}\right) .
$$

Then, the light neutrino mass after seesaw is

$$
\begin{aligned}
-\left(M_{\nu}\right)_{\alpha \beta}= & \frac{\left(m_{D}\right)_{\alpha 1}\left(m_{D}\right)_{\beta 3}+\left(m_{D}\right)_{\alpha 3}\left(m_{D}\right)_{\beta 1}}{M_{1}} \\
& +\frac{\left(m_{D}\right)_{\alpha 2}\left(m_{D}\right)_{\beta 2}}{M_{3}} .
\end{aligned}
$$

The heavy neutrino masses are $M_{1}, M_{2}\left(=-M_{1}\right)$, and $M_{3}$. The light-heavy neutrino mixings are approximately

$$
X_{\alpha 1}=X_{\alpha 2} \simeq \frac{\left(m_{D}\right)_{\alpha 3}}{M_{1}}, \quad X_{\alpha 3} \simeq 0 .
$$

To obtain the proper size of light neutrino masses with sizable light-heavy neutrino mixings, $\left(m_{D}\right)_{\alpha 1}$ needs to be small.

We plot the upper bound of $\left|G_{1}\right| / G_{F}$ in the above setup in Fig. 2. For $M_{1} \lesssim 30 \mathrm{TeV}$, the Dirac mass $\left(m_{D}\right)_{\alpha 3}$ is chosen just to satisfy the $\mu \rightarrow e \gamma$ bound. For $M_{1} \gtrsim 1 \mathrm{TeV}$, the loop function $\tilde{F}$ for $\mu \rightarrow e \gamma$ does not depend on $M_{1}$ very 


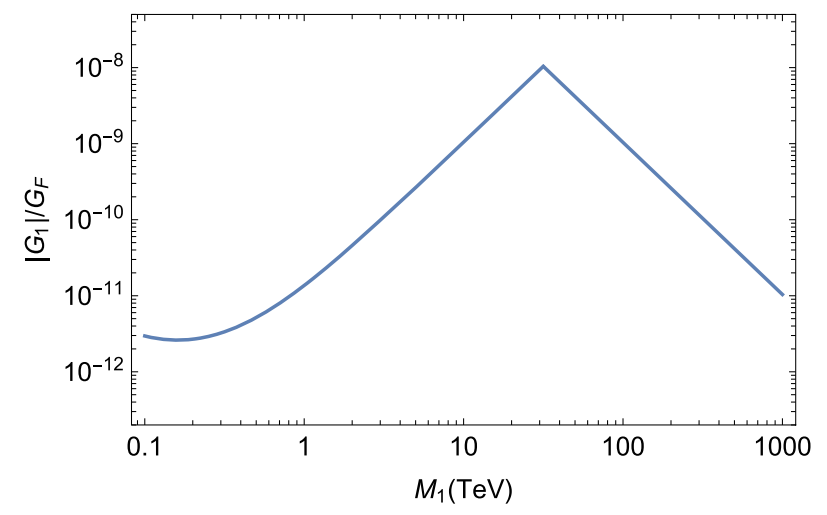

FIG. 2. The upper bound of $\left|G_{1}\right| / G_{F}$ as a function of the heavy neutrino mass. For the neutrino mass to be less than $\sim 30 \mathrm{TeV}$, the $\mathrm{Mu}-$ to- $\overline{\mathrm{Mu}}$ transition is bounded by the $\mu \rightarrow e \gamma$ constraint.

much (due to the longitudinal modes of the gauge bosons in the unitary gauge), and thus the maximal value of $\left|X_{e 1} X_{\mu 1}\right|$ does not depend on $M_{1}$. Therefore, in the region of $1 \mathrm{TeV} \lesssim M_{1} \lesssim 30 \mathrm{TeV}$, the upper bound of the Mu-to- $\overline{\mathrm{Mu}}$ transition behaves as $\left|G_{1}\right| \propto M_{1}^{2}$ because of $4 E_{0} \sim M_{1}^{2} / M_{W}^{2}$ for $M_{1} \gg M_{W}$. For $M_{1} \gtrsim 30 \mathrm{TeV}$, the $\mu \rightarrow e \gamma$ bound can satisfy for $\left(m_{D}\right)_{\alpha 3}<100 \mathrm{GeV}$, and then, the upper bound behaves as $\left|G_{1}\right| \propto 1 / M_{1}^{2}$.

\section{TYPE-II SEESAW MODEL}

The doubly charged scalar can couple with two charged leptons, and the Mu-to- $\overline{\mathrm{Mu}}$ transition can be induced by the exchange of it at the tree level [31,32] as shown in Fig. 3. The doubly charged scalar which can couple to right-handed charged leptons is a $S U(2)_{L}$ singlet with hypercharge $Y=2$, while a $S U(2)_{L}$ triplet scalar with hypercharge $Y=1$ can couple to the left-handed lepton doublets $\ell$.

In the type-II seesaw model [33-36], the neutrino masses are generated by a vacuum expectation value (vev) of the neutral component of the $S U(2)_{L}$ triplet scalar:

$$
\left(\Delta_{L}\right)_{a b}=\left(\begin{array}{cc}
\Delta_{L}^{++} & \Delta_{L}^{+} / \sqrt{2} \\
\Delta_{L}^{+} / \sqrt{2} & \Delta_{L}^{0}
\end{array}\right) .
$$

Therefore, the neutrino mass generation in the type-II seesaw model can be related to the Mu-to- $\overline{\mathrm{Mu}}$ transition [37].

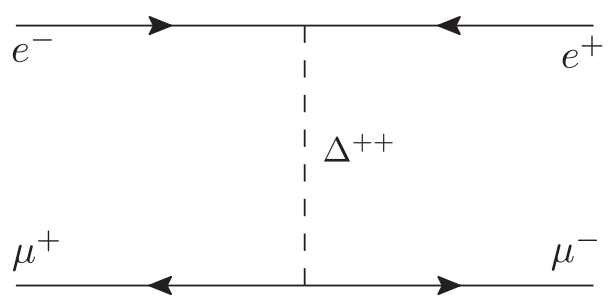

FIG. 3. The tree-level exchange of a doubly charged scalar boson $\Delta^{++}$to induce the $\mathrm{Mu}-\mathrm{to}-\overline{\mathrm{Mu}}$ transition.
The Lagrangian of the type-II seesaw can be written as

$$
\begin{aligned}
-\mathcal{L}= & \left(\frac{1}{2} \kappa_{i j}^{L} \overline{\left(\ell_{i L}\right)^{c}} \ell_{j L} \Delta_{L}+\mu_{\Delta} H H \Delta_{L}^{*}+\text { H.c. }\right) \\
& +M_{\Delta}^{2}\left|\Delta_{L}\right|^{2},
\end{aligned}
$$

where $H$ is a Higgs doublet with hypercharge $Y=1 / 2$, and $\mu_{\Delta}$ is a dimensionful scalar trilinear coupling. The " $\ell \ell \Delta_{L}$ " term is written down as

$$
\begin{aligned}
\overline{\left(\ell_{i L}\right)^{c}} \ell_{j L} \Delta_{L}= & \overline{\nu_{i L}^{c}} \nu_{j L} \Delta_{L}^{0}-\frac{1}{\sqrt{2}} \overline{\nu_{i L}^{c}} e_{j L} \Delta_{L}^{+} \\
& -\frac{1}{\sqrt{2}} \overline{e_{i L}^{c}} \nu_{j L} \Delta_{L}^{+}+\overline{e_{i L}^{c}} e_{j L} \Delta_{L}^{++} .
\end{aligned}
$$

By integrating out $\Delta_{L}$, the dimension-five neutrino mass operator (so-called Weinberg operator, " $\ell \ell H H$ ") can be generated, which can be also interpreted to mean that the vev of $\Delta_{L}^{0}$ is $v_{L} \equiv\left\langle\Delta_{L}^{0}\right\rangle=-\mu_{\Delta}\left\langle H^{0}\right\rangle^{2} / M_{\Delta}^{2}$, and the type-II neutrino mass is

$$
M_{\nu}^{\mathrm{II}}=\kappa^{L} v_{L}
$$

As mentioned, the type-II seesaw Lagrangian contains the doubly charged scalar couplings to the left-handed charged leptons. We here describe it using two-component spinor convention to avoid the complication of the expressions, ${ }^{3}$

$-\mathcal{L} \supset \frac{1}{2} \kappa_{i j}^{L} \mathrm{e}_{i} \mathrm{e}_{j} \Delta_{L}^{++}+\frac{1}{2} \kappa_{i j}^{L *} \overline{\mathrm{e}}_{i} \overline{\mathrm{e}}_{j} \Delta_{L}^{--}+M_{\Delta}^{2} \Delta_{L}^{--} \Delta_{L}^{++}$,

where e denotes the two-component spinor. Integrating out $\Delta_{L}^{++}=\left(\Delta_{L}^{--}\right)^{*}$ by equation of motion, $1 / 2 \kappa_{i j}^{L} \mathrm{e}_{i} \mathrm{e}_{j}+$ $M_{\Delta}^{2} \Delta^{--}=0$, one obtains

$$
-\mathcal{L} \supset-\frac{1}{4} \frac{1}{M_{\Delta}^{2}} \kappa_{i j}^{L} \kappa_{k l}^{L *}\left(\mathrm{e}_{i} \mathrm{e}_{j}\right)\left(\overline{\mathrm{e}}_{k} \overline{\mathrm{e}}_{l}\right) .
$$

Using

$$
\delta_{\beta}^{\alpha} \delta_{\dot{\alpha}}^{\dot{\beta}}=\frac{1}{2} \sigma_{\beta \dot{\alpha}}^{\mu} \bar{\sigma}_{\mu}^{\dot{\beta} \alpha}
$$

one finds

$$
\mathrm{e}_{i} \mathrm{e}_{j}=\overline{e_{i}^{c}} P_{L} e_{j}=e_{i}^{T} C P_{L} e_{j}
$$

In the chiral representation, the four-component spinor can be expressed as

$e=\left(\begin{array}{c}\mathrm{e} \\ \overline{\mathrm{e}^{\mathrm{c}}}\end{array}\right), \quad e^{c}=\left(\begin{array}{c}\mathrm{e}^{\mathrm{c}} \\ \overline{\mathrm{e}}\end{array}\right), \quad \bar{e}=\left(\begin{array}{ll}\mathrm{e}^{c} & \overline{\mathrm{e}}\end{array}\right), \quad \overline{e^{c}}=\left(\mathrm{e} \overline{\mathrm{e}^{\mathrm{c}}}\right)$. 


$$
\left(\mathrm{e}_{i} \mathrm{e}_{j}\right)\left(\overline{\mathrm{e}}_{k} \overline{\mathrm{e}}_{l}\right)=\frac{1}{2}\left(\overline{\mathrm{e}}_{l} \bar{\sigma}^{\mu} \mathrm{e}_{i}\right)\left(\overline{\mathrm{e}}_{k} \bar{\sigma}_{\mu} \mathrm{e}_{j}\right) .
$$

Expressing it in four-component fermion convention, we obtain

$$
-\mathcal{L} \supset-\frac{1}{8} \frac{1}{M_{\Delta}^{2}} \kappa_{i j}^{L} \kappa_{k l}^{L *}\left(\overline{e_{l}} \gamma^{\mu} P_{L} e_{i}\right)\left(\overline{e_{k}} \gamma_{\mu} P_{L} e_{j}\right)
$$

and the coefficient of the transition operator can be written as

$$
\frac{G_{1}}{\sqrt{2}}=-\frac{\kappa_{e e}^{L} \kappa_{\mu \mu}^{L *}}{32 M_{\Delta}^{2}}=-\frac{1}{32 v_{L}^{2} M_{\Delta}^{2}}\left(M_{\nu}^{\mathrm{II}}\right)_{e e}\left(M_{\nu}^{\mathrm{II}}\right)_{\mu \mu}^{*} .
$$

The four-Fermi operator Eq. (5.11) can generate LFV decays

$$
\begin{aligned}
\operatorname{Br} & \left(l_{a}^{-} \rightarrow l_{b}^{+} l_{c}^{-} l_{d}^{-}\right) \\
& =\frac{1}{2\left(1+\delta_{c d}\right)}\left|\frac{\kappa_{a b}^{L} \kappa_{c d}^{L *}}{4 G_{F} M_{\Delta}^{2}}\right|^{2} \times \operatorname{Br}\left(l_{a}^{-} \rightarrow l_{b}^{-} \nu \bar{\nu}\right) .
\end{aligned}
$$

The $\mu \rightarrow 3 e$ decay process gives the most stringent constraint to the $\mathrm{Mu}-\mathrm{to}-\overline{\mathrm{Mu}}$ transition in the model:

$$
\operatorname{Br}(\mu \rightarrow 3 e)<1.0 \times 10^{-12} .
$$

We find

$\frac{\left|G_{1}\right|}{G_{F}}=\sqrt{\operatorname{Br}(\mu \rightarrow 3 e)} \frac{1}{2 \sqrt{2}}\left|\frac{\kappa_{\mu \mu}^{L}}{\kappa_{e \mu}^{L}}\right| \lesssim 3.5 \times 10^{-7}\left|\frac{\kappa_{\mu \mu}^{L}}{\kappa_{e \mu}^{L}}\right|$.

We suppose that the type-II term dominates the active neutrino mass (e.g., there is no right-handed neutrino, or the type-I contribution is negligible for the right-handed neutrinos to be very heavy), and the type-II neutrino mass matrix is

$$
M_{\nu}^{\mathrm{II}}=U_{\mathrm{PMNS}}^{*} \operatorname{diag}\left(m_{1} e^{i \alpha_{1}}, m_{2} e^{i \alpha_{2}}, m_{3}\right) U_{\mathrm{PMNS}}^{\dagger},
$$

where $U_{\text {PMNS }}$ is the Pontecorvo-Maki-Nakagawa-Sakata (PMNS) neutrino mixing matrix given by Particle Data Group convention [38], and $m_{i}$ 's are the active neutrino masses. Naively, one obtains

$$
\frac{\kappa_{e \mu}^{L}}{\kappa_{\mu \mu}^{L}} \sim O\left(\theta_{13}, \sqrt{\Delta m_{\mathrm{sol}}^{2} / \Delta m_{\mathrm{atm}}^{2}}\right),
$$

and $\left|\kappa_{e \mu}^{L} / \kappa_{\mu \mu}^{L}\right|$ is roughly $10 \%-20 \%$. Then, $\left|G_{1}\right| / G_{F}$ is smaller than $O\left(10^{-6}\right)$, which cannot be observed in near-future experiments. However, the observed neutrino mixings can be realized even if $\kappa_{e \mu}^{L} \rightarrow 0$. From the viewpoints of the masses and mixings, $\kappa_{e \mu}^{L} \rightarrow 0$ can happen if
(1) The neutrino masses are degenerate, $m_{1} e^{i \alpha_{1}} \simeq$ $m_{2} e^{i \alpha_{2}}\left(\simeq m_{3}\right)$.

(2) $\sum U_{e i}^{*} U_{\mu i}^{*} m_{i} e^{i \alpha_{i}}$ is accidentally canceled, which can happen since $\theta_{13} \sim \sqrt{\Delta m_{\mathrm{sol}}^{2} / \Delta m_{\mathrm{atm}}^{2}}$ and $\theta_{23}, \theta_{12} \sim$ $O(1)$.

In the case of the degenerate solution, the transition probability is maximized (for fixed $v_{L}$ and $M_{\Delta}$ ), and then, the half-life of neutrinoless double beta decay can be just above the current bound (if other experimental data allow the solution).

Let us calculate the numerical upper bounds of $\left|G_{1}\right| / G_{F}$ allowed by the constraints from LFV decays [38]:

$$
\{\operatorname{Br}(\tau \rightarrow 3 e), \operatorname{Br}(\tau \rightarrow 3 \mu)\}<\{2.7,2.1\} \times 10^{-8},
$$

$\left\{\operatorname{Br}\left(\tau^{-} \rightarrow e^{+} \mu^{-} \mu^{-}\right), \operatorname{Br}\left(\tau^{-} \rightarrow \mu^{+} e^{-} e^{-}\right)\right\}<\{1.7,1.5\} \times 10^{-8}$.

The LFV decay bounds can give the upper bounds of the $\mathrm{Mu}$-to- $\overline{\mathrm{Mu}}$ transition as

$$
\frac{\left|G_{1}\right|}{G_{F}} \simeq \sqrt{\frac{\operatorname{Br}(\tau \rightarrow 3 e)}{\operatorname{Br}(\tau \rightarrow e \nu \bar{\nu})}} \frac{1}{2 \sqrt{2}}\left|\frac{\kappa_{\mu \mu}^{L}}{\kappa_{e \tau}^{L}}\right| \lesssim 1.4 \times 10^{-4}\left|\frac{\kappa_{\mu \mu}^{L}}{\kappa_{e \tau}^{L}}\right|,
$$

$$
\frac{\left|G_{1}\right|}{G_{F}} \simeq \sqrt{\frac{\operatorname{Br}\left(\tau^{-} \rightarrow \mu^{+} e^{-} e^{-}\right)}{\operatorname{Br}(\tau \rightarrow \mu \nu \bar{\nu})}} \frac{1}{2 \sqrt{2}}\left|\frac{\kappa_{\mu \mu}^{L}}{\kappa_{\mu \tau}^{L}}\right| \lesssim 1.0 \times 10^{-4}\left|\frac{\kappa_{\mu \mu}^{L}}{\kappa_{\mu \tau}^{L}}\right|,
$$

$$
\frac{\left|G_{1}\right|}{G_{F}} \simeq \sqrt{\frac{\operatorname{Br}\left(\tau^{-} \rightarrow e^{+} \mu^{-} \mu^{-}\right)}{\operatorname{Br}(\tau \rightarrow e \nu \bar{\nu})}} \frac{1}{2 \sqrt{2}}\left|\frac{\kappa_{e e}^{L}}{\kappa_{e \tau}^{L}}\right| \lesssim 1.1 \times 10^{-4}\left|\frac{\kappa_{e e}^{L}}{\kappa_{e \tau}^{L}}\right|,
$$

$$
\frac{\left|G_{1}\right|}{G_{F}} \simeq \sqrt{\frac{\operatorname{Br}(\tau \rightarrow 3 \mu)}{\operatorname{Br}(\tau \rightarrow \mu \nu \bar{\nu})}} \frac{1}{2 \sqrt{2}}\left|\frac{\kappa_{e e}^{L}}{\kappa_{e \tau}^{L}}\right| \lesssim 1.2 \times 10^{-4}\left|\frac{\kappa_{e e}^{L}}{\kappa_{e \tau}^{L}}\right| .
$$

The $\mu \rightarrow e \gamma$ (and $\mu-e$ conversion [39]) constraint can be written as ${ }^{4}$

$$
\frac{\left|\kappa_{e \tau}^{L} \kappa_{\mu \tau}^{L *}\right|}{M_{\Delta}^{2}} \lesssim 1 \times 10^{-3} \times \frac{1}{(1 \mathrm{TeV})^{2}},
$$

which is interpreted as

$$
\operatorname{Br}(\mu \rightarrow e \gamma)=\frac{3 \alpha}{16 \pi}\left|\frac{\kappa_{e i}^{L} \kappa_{\mu i}^{L *}}{3 G_{F}}\left(\frac{1}{M_{\Delta^{++}}^{2}}+\frac{1}{8 M_{\Delta^{+}}^{2}}\right)\right|^{2}
$$

The $S U(2)_{L}$ triplet contains a single charged scalar, which contributes $\mu \rightarrow e \gamma$. 

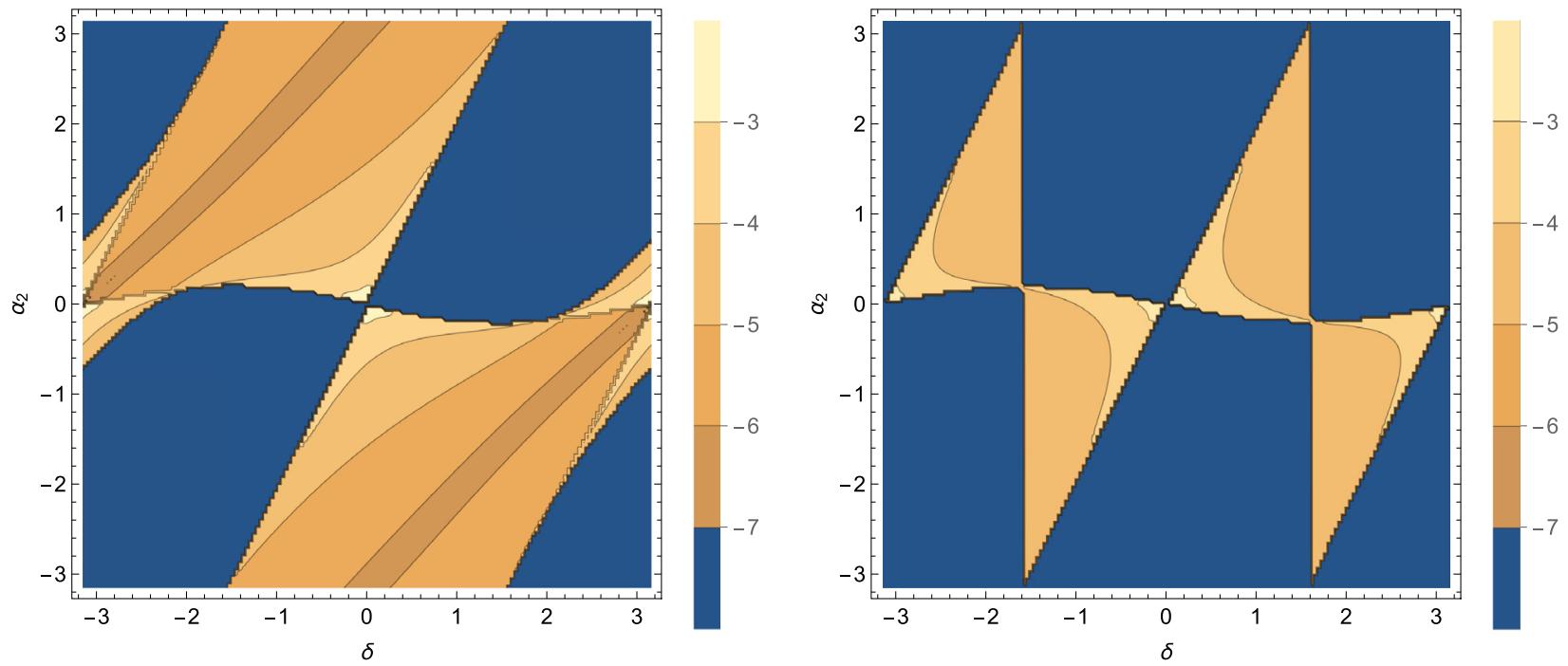

FIG. 4. Contour plots of upper bound of $\log _{10}\left(\left|G_{1}\right| / G_{F}\right)$ from LFV decays as a function of $\delta$ and $\alpha_{2}$ (in radian) for normal mass ordering (left) and inverted mass ordering (right). In the dark blue region, there is no solution to make $\kappa_{e \mu}^{L} \rightarrow 0$.

$$
\frac{\left|G_{1}\right|}{G_{F}}<3.8 \times 10^{-6}\left|\frac{\kappa_{e e}^{L} \kappa_{\mu \mu}^{L}}{\kappa_{e \tau}^{L} \kappa_{\mu \tau}^{L}}\right|
$$

We solve the equation $\kappa_{e \mu}^{L}=0$ by $m_{1} e^{i \alpha_{1}}$ to satisfy the severe $\mu \rightarrow 3 e$ bound. Eliminating $m_{1} e^{i \alpha_{1}}$ from the equation, we obtain the neutrino mass matrix as a function of a Dirac phase $\delta$ in the PMNS matrix and a Majorana phase $\alpha_{2}$. Therefore, the upper bounds of the Mu-to- $\overline{M u}$ transition are obtained as shown in Fig. 4. Surely, the plot in Fig. 4 is symmetric under $\delta \rightarrow-\delta$ and $\alpha_{2} \rightarrow-\alpha_{2}$, because of $G_{1} \rightarrow G_{1}^{*}$. Near $\delta \sim 0, \pi$ (and $\alpha_{2} \sim 0$ ), the degenerate solution can be obtained, and thus, the Mu-to-Mu transition can be largest there. In the inverted hierarchy case, $m_{1}$ and $m_{2}$ are degenerate by themselves, and the Mu-to- $\overline{\mathrm{Mu}}$ transition can be large at all the points (if there is a solution to make $\kappa_{e \mu}^{L} \rightarrow 0$ ). In the normal hierarchy case, $\kappa_{e \mu}^{L}$ can be canceled even without mass degeneracy. Actually, both

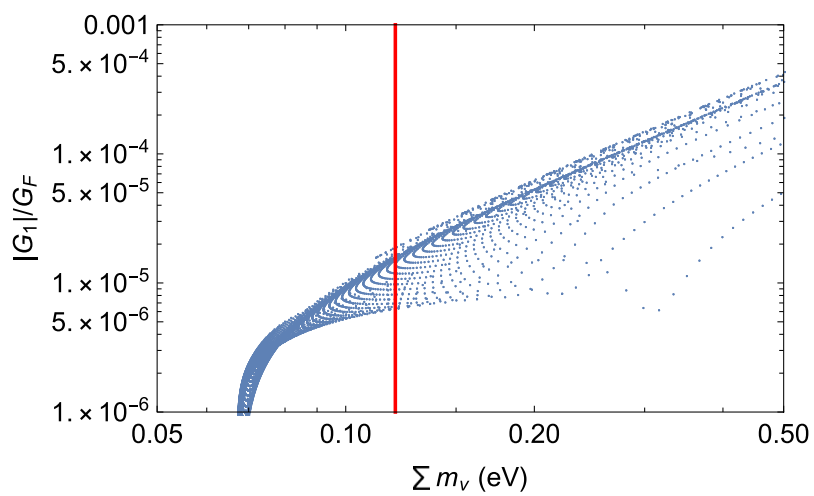

$\kappa_{e e}^{L}$ and $\kappa_{e \mu}^{L}$ can be small to reproduce the neutrino oscillation data. Therefore, there is a band where the $\mathrm{Mu}-\mathrm{to}-\overline{\mathrm{Mu}}$ transition is small in the plot.

The current strongest bound of the absolute neutrino mass is from cosmological measurement: the total neutrino mass $\Sigma m_{\nu}<0.12 \mathrm{eV}$ [40]. Therefore, unless there is a loophole (e.g., the neutrinos are not stable in the cosmological time scale [41]), the solution of the large degree of degeneracy is excluded and the Mu-to- $\overline{\mathrm{Mu}}$ transition is bounded. In Fig. 5, we show the plot of the coefficient of the $\mathrm{Mu}$-to- $\overline{\mathrm{Mu}}$ transition operator versus the total neutrino mass. The shown Mu-to- $\overline{\mathrm{Mu}}$ transition in the plot is the upper bound from LFV as described above, generated by a mesh of $\delta$ and $\alpha_{2}$. The cosmological measurements bound the Mu-to- $\overline{\mathrm{Mu}}$ transition as $\left|G_{1}\right| / G_{F} \lesssim O\left(10^{-5}\right)$.

If we adopt the type-I seesaw contributions in addition to type-II, one can tune $\kappa_{e \mu}^{L}$ to be zero irrespective of the neutrino masses and mixings, and because of the many

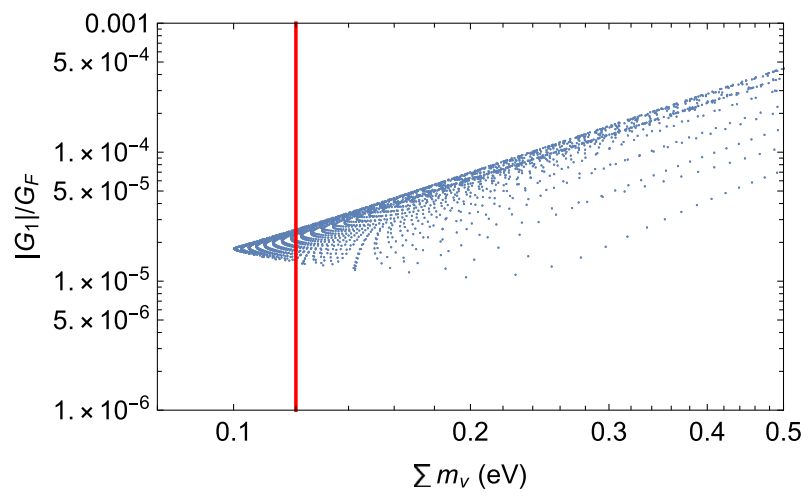

FIG. 5. The plot of $\left|G_{1}\right| / G_{F}$ allowed by LFV constraints versus the total neutrino mass for normal mass ordering (left) and inverted mass ordering (right). The vertical red line shows $\sum m_{\nu}=0.12 \mathrm{eV}$. 
parameters, any size of the Mu-to- $\overline{\mathrm{Mu}}$ transition can be fit satisfying experimental constraints in principle [37]. Supposing $\kappa_{e e}^{L} \sim \kappa_{\mu \mu}^{L} \sim 0.3$, and $M_{\Delta}=600 \mathrm{GeV}$ (the experimental bound of the doubly charged scalar mass can be found in $[42,43])$, one finds

$$
\left|G_{1}\right| \sim 1 \times 10^{-3} G_{F},
$$

which can be soon tested by the near-future experiments.

\section{LEFT-RIGHT MODEL}

The $S U(3)_{c} \times S U(2)_{L} \times S U(2)_{R} \times U(1)_{B-L}\left(\equiv G_{3221}\right)$ gauge theory (left-right model) to induce the Mu-to- $\overline{\mathrm{Mu}}$ transition $[44,45]$ is one of the representative models where the new experimental results in this quarter-century have brought about changes drastically. In the early 1990s, there was still room that the active neutrinos can lie around 10-100 keV. Surely, the neutrino oscillations exclude the room, and a large mixing between left-handed neutrinos and SM singlet right-handed neutrinos at such mass scale is not allowed. The meson mixing data pushes up the $W_{R}$ gauge boson mass to be more than $3 \mathrm{TeV}[46,47]$. The direct LHC data from $W_{R}^{+} \rightarrow N_{R} \ell_{R}^{+} \rightarrow j j \ell_{R}^{ \pm} \ell_{R}^{+}$processes gives the lower bound of the $W_{R}$ mass to be more than $4 \mathrm{TeV}$ [48-50]. Therefore, the resume for the Mu-to- $\overline{\mathrm{Mu}}$ transition in the quarter-century ago is not valid anymore.

Various experimental constraints in the left-right model, especially on the flavor physics, can be found in $[51,52]$. We note that the same sign and opposite sign of the twolepton signals from the $W_{R}^{+} \rightarrow j j \ell_{R}^{ \pm} \ell_{R}^{+}$processes can be a probe of the structure in the neutrino mass matrix, which is related to the degeneracy of the heavy neutrino masses [53].

The Dirac mass of tau neutrino is supposed to be (at least) of the order of $\mathrm{GeV}$ due to left-right symmetry, and thus, one needs fine-tuning to obtain the sub-eV active neutrino mass in the TeV-scale left-right model. Therefore, an extended seesaw model to generate sub-eV active neutrino mass is often considered in the $\mathrm{TeV}$-scale leftright model. We employ three SM singlet fermions $S_{i}$, and consider the neutrino mass as

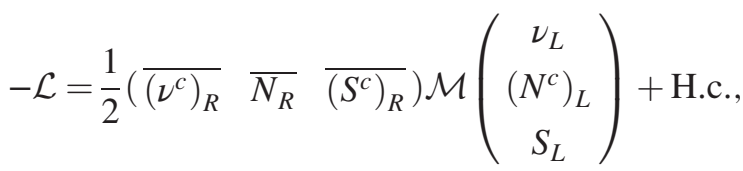

where $\mathcal{M}$ is a $9 \times 9$ mass matrix (in the basis where the charged-lepton mass matrix is diagonal),

$$
\mathcal{M}=\left(\begin{array}{ccc}
0 & m_{D} & 0 \\
m_{D}^{T} & \mu_{N} & M_{S} \\
0 & M_{S}^{T} & \mu_{S}
\end{array}\right)
$$

The light neutrino mass matrix is

$$
\begin{aligned}
M_{\nu}^{\text {light }} & =m_{D}\left(M_{S} \mu_{S}^{-1} M_{S}^{T}-\mu_{N}\right)^{-1} m_{D}^{T} \\
& \simeq m_{D}\left(M_{S}^{T}\right)^{-1} \mu_{S} M_{S}^{-1} m_{D}^{T} .
\end{aligned}
$$

We suppose that the Majorana mass $\mu_{S}$ of the singlet $S$ is small, and then the active neutrino mass can be sub-eV easily even in the $\mathrm{TeV}$-scale left-right model. This is sometimes called an inverse seesaw.

The Dirac mass $m_{D}$ comes from the usual Dirac Yukawa coupling to Higgs bidoublet: $(\mathbf{1}, \mathbf{2}, \mathbf{2}, 0)$ under $G_{3221}$, and $M_{S}$ comes from the $\Phi \overline{\ell_{R}} S_{L}$ coupling with $\Phi:(\mathbf{1}, \mathbf{1}, \mathbf{2},-1)$ under $G_{3221}$. The vev of $\Phi$ breaks $G_{3221}$ down to SM gauge symmetry. The Majorana mass $\mu_{N}$ is generated if there is a $S U(2)_{R}$ triplet $\Delta_{R}:(\mathbf{1}, \mathbf{1}, \mathbf{3}, 2)$ and it acquires a vev to break $G_{3221}$. How the Mu-to-Mu transition is induced in the left-right model depends on with or without the $S U(2)_{R}$ triplet. In the case without the triplet, the Mu-to- $\overline{\mathrm{Mu}}$ transition is generated at the loop level, while in the case with the triplet, it can be generated at the tree level since the triplet contains the doubly charged scalar.

We parametrize ${ }^{5}$

$$
\begin{gathered}
m_{D}=U_{0}^{*} \operatorname{diag}\left(m_{D}^{1}, m_{D}^{2}, m_{D}^{3}\right) V_{0}^{\dagger}, \\
\mu_{N}=V_{1}^{*} \operatorname{diag}\left(\mu_{N}^{1}, \mu_{N}^{2}, \mu_{N}^{3}\right) V_{1}^{\dagger}, \\
M_{S}=V_{2}^{*} \operatorname{diag}\left(M_{S}^{1}, M_{S}^{2}, M_{S}^{3}\right) .
\end{gathered}
$$

The convention in the diagonalization of the neutrino mass matrix is given in Appendix C. The matrix $U$ in the $9 \times 9$ diagonalization matrix in Eq. (C9), which corresponds to nearly the $3 \times 3$ PMNS matrix, is a diagonalization matrix of

$U_{0}^{*} m_{D}^{\text {diag }} V_{0}^{\dagger} V_{2}\left(M_{S}^{\text {diag }}\right)^{-1} \mu_{S}\left(M_{S}^{\text {diag }}\right)^{-1} V_{2}^{T} V_{0}^{*} m_{D}^{\text {diag }} U_{0}^{T}$.

In the left-right model, the mixings in $U_{0}$ and $V_{0}$ are expected to be small as CKM mixings, but the structure of $M_{S}$ and $\mu_{S}$ can have freedom to generate large neutrino mixings. Surely, one can also employ a $S U(2)_{L}$ triplet and consider the type-II seesaw contribution for the active neutrino mass.

\section{A. Case 1: Without $S U(2)_{R}$ triplet}

If there is no $S U(2)_{R}$ triplet, the Mu-to- $\overline{\mathrm{Mu}}$ transition is generated by a box loop diagram. In addition to the $W_{L}-W_{L}$ loop diagram in Fig. 1 , we have $W_{R}-W_{R}$ box loop contributions:

\footnotetext{
${ }^{5}$ Since the singlet $S$ does not have a reference current basis, one can parametrize the matrix $M_{S}$ to be given in Eq. (6.6) without loss of generality and $\mu_{S}$ to be a general $3 \times 3$ matrix.
} 


$$
\begin{aligned}
\frac{G_{2}}{\sqrt{2}}= & \frac{G_{F}^{2} M_{W_{L}}^{2}}{16 \pi^{2}} \frac{g_{R}^{4}}{g_{L}^{4}} \frac{1}{z} \sum_{I, J}\left[Y_{\mu I}^{*} Y_{e I} Y_{\mu J}^{*} Y_{e J} E_{0}\left(\tilde{x}_{I}, \tilde{x}_{J}\right)\right. \\
& \left.+\left(Y_{\mu I}^{*}\right)^{2}\left(Y_{e J}\right)^{2} E_{1}\left(\tilde{x}_{I}, \tilde{x}_{J}\right)\right],
\end{aligned}
$$

and $W_{L}-W_{R}$ box loop contributions:

$$
\begin{aligned}
\frac{G_{3}}{\sqrt{2}}= & -\frac{G_{F}^{2} M_{W_{L}}^{2}}{8 \pi^{2}} \frac{g_{R}^{2}}{g_{L}^{2}} \sum_{I, J}\left[X_{\mu I} Y_{\mu I}^{*} X_{e J}^{*} Y_{e J} E_{0}\left(x_{I}, x_{J}, z\right)\right. \\
& \left.+X_{\mu I} Y_{e I} X_{e J}^{*} Y_{\mu J}^{*} E_{1}\left(x_{I}, x_{J}, z\right)\right],
\end{aligned}
$$

where

$$
\tilde{x}_{I}=\frac{M_{N_{I}}^{2}}{M_{W_{R}}^{2}}, \quad x_{I}=\frac{M_{N_{I}}^{2}}{M_{W_{L}}^{2}}, \quad z=\frac{M_{W_{R}}^{2}}{M_{W_{L}}^{2}} .
$$

The loop functions $E_{0}$ and $E_{1}$ are given in Appendix D. Strictly speaking, since there is a $W_{L}-W_{R}$ mixing due to vevs of Higgs bidoublet, the mass eigenstates should be quoted as their mixed states. We here neglect their mixing in the box contributions.

One can find that $E_{1}$ term in $G_{2}$ and $E_{0}$ term in $G_{3}$ correspond to the Mu-to- $\overline{\mathrm{Mu}}$ transition utilized by the Majorana property of the heavy neutrinos. If there is no $S U(2)_{R}$ triplet, the Majorana mass of the right-handed neutrino $\mu_{N}$ is absent, and the heavy neutrino masses are degenerate in the setup of the inverse seesaw. Then, their contributions are canceled. Therefore, our concerns are $E_{0}$ term in $G_{2}$ and $E_{1}$ term in $G_{3}$, which are bounded by $\mu \rightarrow e \gamma$. The $\mu_{R} \rightarrow e_{L} \gamma$ amplitude via $W_{L}$ loop is

$$
A_{R}\left(W_{L}\right)=\frac{e m_{\mu}}{16 \pi^{2}} \frac{G_{F}}{\sqrt{2}} \sum_{I} X_{\mu I}^{*} X_{e I} \tilde{F}\left(x_{I}\right),
$$

where $\tilde{F}$ is given in Eq. (4.7), and the $\mu_{L} \rightarrow e_{R} \gamma$ amplitude via $W_{R}$ loop is

$$
A_{L}\left(W_{R}\right)=\frac{e m_{\mu}}{16 \pi^{2}} \frac{G_{F}}{\sqrt{2}} \frac{g_{R}^{2}}{g_{L}^{2}} \frac{1}{z} \sum_{I} Y_{\mu I} Y_{e I}^{*} \tilde{F}\left(\tilde{x}_{I}\right)
$$

Because there is a $W_{L}-W_{R}$ mixing $\xi_{L R}$, the chirality can flip at the internal line in the loop and the decay amplitudes are

$$
\begin{aligned}
& A_{R}\left(W_{L}-W_{R}\right) \\
& =\frac{e}{16 \pi^{2}} \xi_{L R} \frac{g_{R}}{g_{L}} \frac{G_{F}}{\sqrt{2}} \sum_{I} Y_{\mu I} X_{e I} M_{N_{I}}\left(G\left(x_{I}\right)-\frac{1}{z} G\left(\tilde{x}_{I}\right)\right), \\
& A_{L}\left(W_{L}-W_{R}\right) \\
& \quad=\frac{e}{16 \pi^{2}} \xi_{L R} \frac{g_{R}}{g_{L}} \frac{G_{F}}{\sqrt{2}} \sum_{I} X_{\mu I}^{*} Y_{e I}^{*} M_{N_{I}}\left(G\left(x_{I}\right)-\frac{1}{z} G\left(\tilde{x}_{I}\right)\right),
\end{aligned}
$$

where

$$
G(x)=\frac{2\left(4-15 x+12 x^{2}-x^{3}-6 x^{2} \ln x\right)}{(1-x)^{3}} .
$$

The $\mu \rightarrow e \gamma$ experimental result implies

$$
\begin{aligned}
& \left|\sum_{I} X_{\mu I}^{*} X_{e I} \tilde{F}\left(x_{I}\right)\right|, \\
& \frac{g_{R}^{2}}{g_{L}^{2}} \frac{1}{z}\left|\sum_{I} Y_{\mu I} Y_{e I}^{*} \tilde{F}\left(\tilde{x}_{I}\right)\right| \lesssim 4 \times 10^{-5},
\end{aligned}
$$

if we assume that there is no cancellation in each $A_{L}$ and $A_{R}$. These two constraints restrict the Mu-to- $\overline{\mathrm{Mu}}$ transition operators from $W_{L}-W_{L}$ box and $W_{R}-W_{R}$ box diagrams, respectively. The $\mu \rightarrow e \gamma$ bound via the $W_{L}-W_{R}$ mixing is written as

$$
\begin{aligned}
& \left|\sum_{I} Y_{\mu I}^{*} X_{e I}^{*} M_{N_{I}} G\left(x_{I}\right)\right|, \\
& \left|\sum_{I} Y_{e I}^{*} X_{\mu I}^{*} M_{N_{I}} G\left(x_{I}\right)\right| \lesssim 40 \mathrm{MeV} \times \frac{g_{L}}{g_{R}} \times \frac{10^{-4}}{\xi_{L R}},
\end{aligned}
$$

which restricts the Mu-to- $\overline{\mathrm{Mu}}$ transition from the $W_{L}-W_{R}$ box diagram. Because of

$$
\sum_{I} X_{\alpha I}^{*} Y_{\beta I}^{*} M_{N_{I}} \simeq\left(m_{D}\right)_{\alpha \beta}
$$

those roughly correspond to the bounds of the $e \mu$ and $\mu e$ elements of the Dirac neutrino mass matrix. We remark that the restriction to the Mu-to- $\overline{\mathrm{Mu}}$ transition via $W_{L}-W_{R}$ box $\left(G_{3}\right)$ is severer due to the internal chirality flipping in the $\mu \rightarrow$ e $\gamma$ diagram.

In the case without a $S U(2)_{R}$ triplet, the Majorana mass $\mu_{N}=0$ at the tree level. The flavor violation of the righthanded neutrino is characterized by $V_{2}$ in Eq. (6.6). To show the evaluation of the size of the Mu-to- $\overline{M u}$ transition, we assume $M_{S}^{1} \simeq M_{S}^{2}$ and $\left(V_{2}\right)_{13}=\left(V_{2}\right)_{23}=0$ (so that $M_{S}^{3}$ does not contribute). In Fig. 6, we show the upper bound of the Mu-to- $\overline{\mathrm{Mu}}$ transition by the $W_{R}$ box loop allowed by $\mu \rightarrow e \gamma$ constraint. We suppose $g_{L}=g_{R}$ in the plot. When $M_{N}\left(=M_{S}^{1}\right)$ is fixed, the $\mu \rightarrow e \gamma$ bound to the mixing $\left(V_{2}\right)_{12}$ is relaxed for a heavier $W_{R}$. The upper bound of the $\mathrm{Mu}$-to-Mu transition becomes the largest for a mass of $W_{R}$ just when the maximal mixing is allowed. The largest upper bound (for fixed $M_{N}$ ) becomes larger for larger $M_{N}$ because of the behavior of the box loop function. The mass $M_{N}$ comes from $\Phi \bar{\ell}_{R} S_{L}$ coupling and the vev of $\Phi$ gives $W_{R}$ mass. Therefore, the mass $M_{N}$ should not be much larger than $M_{W_{R}}$, and the bound of the Mu-to- $\overline{\mathrm{Mu}}$ transition is estimated as $\left|G_{2}\right| / G_{F} \lesssim O\left(10^{-8}\right)$. 


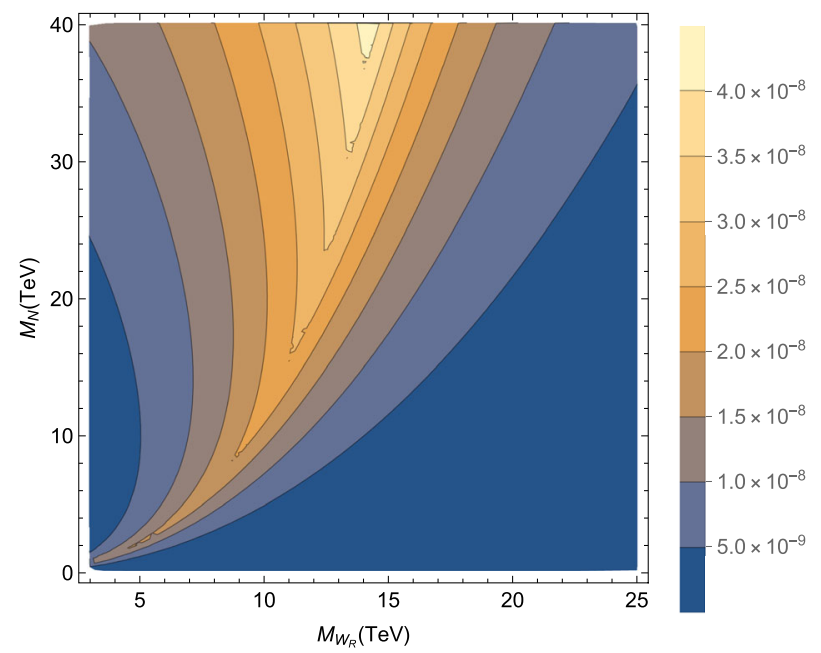

FIG. 6. The upper bound of $\left|G_{2}\right| / G_{F}$ as a function of $M_{W_{R}}$ and the heavy neutrino mass $M_{N}$.

As we have remarked, the $\mu \rightarrow e \gamma$ bound is stronger in the case of internal chirality flipping. The Mu-to- $\overline{\mathrm{Mu}}$ transition via $W_{L}-W_{R}$ diagram is bounded to be $\left|G_{3}\right| / G_{F} \lesssim$ $O\left(10^{-10}\right)$ as long as $\xi_{L R} \gtrsim 0.01 M_{W_{L}}^{2} / M_{W_{R}}^{2}$. We note that the $W_{L}-W_{R}$ mixing $\xi_{L R}$ is proportional to $M_{W_{L}}^{2} / M_{W_{R}}^{2}$, and the proportionality coefficient is determined by the ratio of the vevs of Higgs bidoublet, though we do not describe it in detail in this paper.

\section{B. Case 2: With $S U(2)_{R}$ triplet}

If there is $S U(2)_{R}$ triplet Higgs to break $S U(2)_{R} \times$ $U(1)_{B-L}$, Majorana masses of the right-handed neutrinos can be generated:

$$
\begin{gathered}
-\mathcal{L} \supset \frac{1}{2} \kappa_{i j}^{R} \overline{\ell_{i R}}\left(\ell_{j}^{c}\right)_{L} \Delta_{R}+\text { H.c. }, \\
\mu_{N}=\kappa^{R}\left\langle\Delta_{R}^{0}\right\rangle .
\end{gathered}
$$

The box loop contribution can be larger than in case 1. In this case, however, the coupling to induce the Majorana mass can generate the transition operator at the tree level, which can be surely larger than the box loop:

$$
-\mathcal{L} \supset-\frac{1}{8} \frac{1}{M_{\Delta}^{2}} \kappa_{i j}^{R} \kappa_{k l}^{R *}\left(\bar{e}_{i} \gamma^{\mu} P_{R} e_{l}\right)\left(\overline{e_{j}} \gamma_{\mu} P_{R} e_{k}\right),
$$

and

$$
\frac{G_{2}}{\sqrt{2}}=-\frac{\kappa_{e e}^{R *} \kappa_{\mu \mu}^{R}}{32 M_{\Delta}^{2}}
$$

When we parametrize the Majorana mass matrix as

$$
\mu_{N}=\tilde{V} \operatorname{diag}\left(\mu_{N 1}, \mu_{N 2}, \mu_{N 3}\right) \tilde{V}^{T},
$$

the $\kappa^{R}$ matrix is written as

$$
\kappa_{\alpha \beta}^{R}=\tilde{V}_{\alpha I} \tilde{V}_{\beta I} \mu_{N I}
$$

The $\mu \rightarrow 3 e$ bound, $\operatorname{Br}(\mu \rightarrow 3 e)<1.0 \times 10^{-12}$, restricts the $\mathrm{Mu}$-to- $\overline{\mathrm{Mu}}$ transition similarly to the previous,

$$
\frac{\left|G_{2}\right|}{G_{F}} \lesssim 10^{-6} \times \frac{1}{2 \sqrt{2}}\left|\frac{\kappa_{\mu \mu}^{R}}{\kappa_{e \mu}^{R}}\right| .
$$

There are three ways to suppress $\kappa_{e \mu}^{R}=\tilde{V}_{e 1} \tilde{V}_{\mu 1} \mu_{N 1}+$ $\tilde{V}_{e 2} \tilde{V}_{\mu 2} \mu_{N 2}+\tilde{V}_{e 3} \tilde{V}_{\mu 3} \mu_{N 3}$.

(1) The mixings are small: $\tilde{V} \simeq \mathbf{1}$.

(2) The right-handed neutrino masses are degenerate: $\mu_{N 1} \simeq \mu_{N 2}$.

(3) The mixings are not small, and the masses are not degenerate, but the $\kappa_{e \mu}^{R}$ is accidentally canceled by the $\mu_{N 3}$ contribution.

In models with "left-right parity" (exchange symmetry $\left.\ell_{L} \leftrightarrow\left(\ell_{R}\right)^{c}\right)$, one obtains

$$
\kappa^{L}=\kappa^{R},
$$

and Yukawa matrices are symmetric. Therefore, in the case of the type-II dominance $\left(\mu_{S} \rightarrow 0\right), \kappa^{R}$ is also related to the neutrino masses and mixings:

$\kappa^{R} \propto M_{\nu}=U_{\mathrm{PMNS}}^{*} \operatorname{diag}\left(m_{1} e^{i \alpha_{1}}, m_{2} e^{i \alpha_{2}}, m_{3}\right) U_{\mathrm{PMNS}}^{\dagger}$,

and the Mu-to- $\overline{\mathrm{Mu}}$ transition is estimated in parallel to the analysis in the type-II seesaw.

In general, there is no reason that $\tilde{V}_{e 2}$ and $\tilde{V}_{\mu 1}$ are small in the model construction in the left-right model. Rather, the mixing is not small in the unification scenarios, and the $\mathrm{Mu}$-to- $\overline{\mathrm{Mu}}$ transition is much smaller than the near-future experimental reach. If we do not go beyond the left-right symmetry, a global discrete flavor symmetry to suppress LFV can be assigned in the lepton sector and $\tilde{V} \simeq \mathbf{1}$. (Large neutrino mixings can originate from a hidden sector with singlet fermions, where the discrete symmetry is broken.) The right-handed neutrinos (more precisely, mass eigenstates of the heavy neutrinos from $N_{R}$ and $S$ for $\mu_{N} \sim M_{S}$ ), as well as $W_{R}$ gauge boson, should be heavier than $4-5 \mathrm{TeV}$ to satisfy the bound from the $W_{R}^{+} \rightarrow N_{R} \ell_{R}^{+} \rightarrow j j \ell_{R}^{ \pm} \ell_{R}^{+}$ processes at the LHC [48-50]. The doubly charged scalar mass, on the other hand, can be around $1 \mathrm{TeV}[42,43]$, and thus, the Mu-to- $\overline{\mathrm{Mu}}$ transition with $\left|G_{2}\right| / G_{F} \sim 10^{-3}$ can be obtained, which can be tested by the near-future Mu-to- $\overline{\mathrm{Mu}}$ transition experiments along with the direct search at highluminosity LHC experiment. 


\section{RADIATIVE NEUTRINO MASS}

There are plenty of models in which the neutrino masses are induced radiatively. The models can be roughly classified into two groups.

(1) There is no SM singlet fermion.

(2) There are SM singlet fermions, but the Dirac neutrino Yukawa coupling, $\mathrm{NH} \ell$, is forbidden by a discrete symmetry.

The representative model for (1) is called Zee-Babu model [10-13,54-56]. The improved version of the model has a hypercharge $\pm 2, S U(2)_{L}$ singlet scalar, which is a doubly charged scalar and can be a mediator to induce the transition operator. Neutrino masses are generated at the two-loop level. The model where the neutrino masses are induced at a three-loop level is also considered (so-called cocktail model) [57,58].

In the models for (2), the tree-level active neutrino masses are forbidden by discrete symmetries. Because the discrete symmetries can be exploited, the models are often discussed together with dark matter candidates [59]. As we have studied in Sec. IV, the enlargement of the $\mathrm{Mu}-\mathrm{to}-\overline{\mathrm{Mu}}$ transition from the Majorana property suffers from the natural neutrino mass hierarchy due to the lightheavy neutrino mixings induced by the Dirac neutrino masses. Because of the absence of the Dirac neutrino mass, the models are also suitable to discuss the $\mathrm{Mu}$-to- $\overline{\mathrm{Mu}}$ transition from the Majorana property. The model for (2) has a Yukawa coupling $N \eta \ell\left(=N \eta^{+} e_{L}-N \eta^{0} \nu_{L}\right)$ to generate the neutrino mass at the one-loop level, where the neutral component of the $S U(2)_{L}$ doublet $\eta$ does not acquire a vev ( $\eta$ is often called an inert Higgs doublet). Alternatively, the model has a $N S^{+} e_{R}$ type coupling $\left(S^{+}\right.$is a hypercharge $+1 S U(2)_{L}$ singlet scalar), and the neutrino masses are generated at the three-loop level $[58,60,61]$. The $N \eta^{+} e_{L}$ and $N S^{+} e_{R}$ couplings can induce the transition operators via box diagrams.

\section{A. Models with doubly charged scalar}

\section{Zee-Babu model}

In the Zee-Babu model, there are $S U(2)_{L}$ singlet scalars, $h^{+}$and $k^{++}$, with hypercharge $Y=1$ and $Y=2$, respectively. The couplings to the leptons and the masses of the scalars are given as

$$
\begin{aligned}
-\mathcal{L} \supset & \left(f_{i j} \overline{\ell_{i}^{c}} \cdot \ell_{j} h^{+}+g_{i j} \overline{e_{i}} e_{j}^{c} k^{--}+\mu_{h h k} h^{+} h^{+} k^{--}+\text {H.c. }\right) \\
& +m_{h}^{2} h^{-} h^{+}+m_{k}^{2} k^{--} k^{++},
\end{aligned}
$$

where "." stands for the contraction of the $S U(2)_{L}$ doublet: $A \cdot B \equiv \epsilon_{a b} A_{a} B_{b}=A_{1} B_{2}-A_{2} B_{1}$. The coupling matrix $f$ is antisymmetric under the flavor index, and $g$ is symmetric. The scalar trilinear coupling $\mu_{h h k}$ violates the lepton number symmetry.

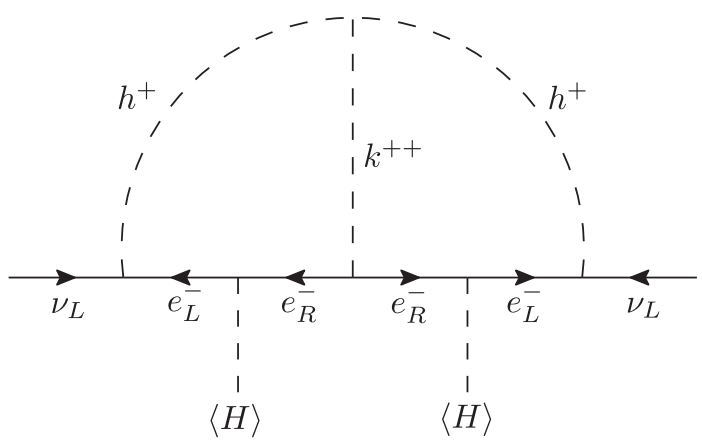

FIG. 7. The diagram to induce the neutrino masses in the ZeeBabu model. The symbol $\langle H\rangle$ stands for the vev of the SM Higgs boson.

The neutrino mass can be induced by two-loop diagram in Fig. 7, and the mass matrix is given as

$$
M_{\nu}=\frac{1}{M_{0}} f M_{e} g M_{e} f^{T}
$$

where $M_{e}=\operatorname{diag}\left(m_{e}, m_{\mu}, m_{\tau}\right)$,

$$
\frac{1}{M_{0}}=\frac{\mu_{h h k}}{48 \pi^{2} \max \left(m_{h}^{2}, m_{k}^{2}\right)} \tilde{I},
$$

and the loop function is approximately given as [54-56]

$$
\tilde{I} \simeq \begin{cases}1 & \text { for } m_{k} \ll m_{h}, \\ 1+\frac{3}{\pi^{2}}\left(\ln ^{2} \frac{m_{k}^{2}}{m_{h}^{2}}-1\right) & \text { for } m_{k} \gg m_{h} .\end{cases}
$$

Because $f$ is anti-symmetric, the neutrino mass matrix is rank 2 (i.e., $m_{1}=0$ ), and the neutrino mass matrix in the normal mass hierarchy is given by the PMNS matrix $U$ as

$M_{\nu}=U^{*} \operatorname{diag}\left(0, m_{2}, m_{3}\right) U^{\dagger}=m_{2} u_{2}^{*} u_{2}^{\dagger}+m_{3} u_{3}^{*} u_{3}^{\dagger}$,

where $m_{i}$ 's are the active neutrino masses as used throughout this paper, and $u_{2}$ and $u_{3}$ are column vectors in $U=\left(u_{1}, u_{2}, u_{3}\right)$ :

$$
\begin{aligned}
& u_{1}=\left(\begin{array}{c}
c_{12} c_{13} \\
-s_{12} c_{23}-e^{i \delta} c_{12} s_{13} s_{23} \\
s_{12} s_{23}-e^{i \delta} c_{12} s_{13} c_{23}
\end{array}\right), \\
& u_{2}=\left(\begin{array}{c}
s_{12} c_{13} \\
c_{12} c_{23}-e^{i \delta} s_{12} s_{13} s_{23} \\
-c_{12} s_{23}-e^{i \delta} s_{12} s_{13} c_{23}
\end{array}\right), \\
& u_{3}=\left(\begin{array}{c}
e^{-i \delta} s_{13} \\
c_{13} s_{23} \\
c_{13} c_{23}
\end{array}\right) .
\end{aligned}
$$


We parametrize the antisymmetric matrix $f$ as

$$
f=\left(\begin{array}{ccc}
0 & f_{3} & -f_{2} \\
-f_{3} & 0 & f_{1} \\
f_{2} & -f_{1} & 0
\end{array}\right),
$$

and then,

$$
f f^{\dagger}=\left|v_{f}\right|^{2} I-v_{f}^{*} v_{f}^{T},
$$

where $v_{f}$ is a column vector, $v_{f}=\left(f_{1}, f_{2}, f_{3}\right)^{T}$, and $I$ is an identity matrix. Suppose that $v_{f}$ is orthogonal to $u_{2}$ and $u_{3}$, i.e., $v_{f}^{T} u_{2}^{*}=v_{f}^{T} u_{3}^{*}=0$, which means that $v_{f}=f_{0} u_{1}\left(f_{0}\right.$ is a coefficient). Then, one finds

$$
f f^{\dagger} M_{\nu} f^{*} f^{T}=\left|f_{0}\right|^{4} M_{\nu},
$$

and

$$
f u_{1}=0 .
$$

One can also obtain

$$
f^{\dagger} u_{2}^{*}=-f_{0}^{*} u_{3}, \quad f^{\dagger} u_{3}^{*}=f_{0}^{*} u_{2} .
$$

Therefore, we find that the solution of Eq. (7.2) is

$$
f=f_{0}\left(\begin{array}{ccc}
0 & U_{\tau 1} & -U_{\mu 1} \\
-U_{\tau 1} & 0 & U_{e 1} \\
U_{\mu 1} & -U_{e 1} & 0
\end{array}\right),
$$

and

$$
\begin{aligned}
\frac{f_{0}^{2}}{M_{0}} M_{e} g M_{e}= & m_{2} u_{3} u_{3}^{T}+m_{3} u_{2} u_{2}^{T}+a_{1} u_{1} u_{1}^{T} \\
& +a_{2}\left(u_{1} u_{2}^{T}+u_{2} u_{1}^{T}\right) \\
& +a_{3}\left(u_{1} u_{3}^{T}+u_{3} u_{1}^{T}\right),
\end{aligned}
$$

where $a_{i}$ 's are arbitrary coefficients with mass dimension. Because any vectors can be given by a linear combination of $u_{i}$, there are three free complex parameters $a_{i}$ (and one parameter $f_{0}$ in $f$ ) in the solution.

Roughly, we obtain (supposing $a_{i}=0$ )

$$
\frac{f_{0}^{2} m_{\mu}^{2} g_{\mu \mu}}{M_{0}} \sim\left(U_{\mu 2}\right)^{2} \sqrt{\Delta m_{\mathrm{atm}}^{2}},
$$

and the size of the scalar mass is estimated as

$$
\frac{M_{0}}{48 \pi^{2}}=\frac{\max \left(m_{h}^{2}, m_{k}^{2}\right)}{\mu_{h h k} \tilde{I}} \sim 420 \mathrm{GeV} \times \frac{f_{0}^{2} g_{\mu \mu} /\left(U_{\mu 2}\right)^{2}}{10^{-3}} .
$$

The coupling $f$ can generate $\mu \rightarrow e \gamma$ process by $f_{e \tau} f_{\mu \tau}^{*}$ product:

$$
\operatorname{Br}(\mu \rightarrow e \gamma)=\frac{3 \alpha}{16 \pi}\left|\frac{f_{e \tau} f_{\mu \tau}^{*}}{3 G_{F} m_{h}^{2}}\right|^{2}
$$

and therefore, the magnitude of $f_{0}$ is bounded to satisfy the $\mu \rightarrow e \gamma$ experimental constraint: roughly, $\left|f_{0}\right|^{2} \lesssim$ $0.002 \times\left(m_{h} / 1 \mathrm{TeV}\right)^{2}$.

Since there are three free parameters $a_{i}$, one can eliminate all off-diagonal elements of the coupling matrix $g$ to suppress the LFV three-body decays of charged leptons. In that case, however, $g_{e e}$ becomes larger than 1 since $g_{e e} m_{e}^{2} \sim g_{\mu \mu} m_{\mu}^{2}$. Therefore, using one degree of freedom, we need to adjust the $e e$ element of $M_{e} g M_{e}$. Then, one of the three off-diagonal elements of $g$ cannot be eliminated. Because it is expected that $g_{\mu \tau}$ is small $\left(g_{\mu \tau} \simeq g_{\mu \mu} m_{\mu} / m_{\tau}\right), \tau \rightarrow 3 \mu$ bound can be satisfied and the other bounds of LFV processes can be satisfied by eliminating $e \mu$ and $e \tau$ elements of $M_{e} g M_{e}$ using the remaining two degrees of freedom.

In Fig. 8 (left), we show the contour plot of $\left|G_{2}\right| / G_{F}$ as a function of the Dirac phase $\delta$ and the Majorana phase $\alpha_{2}$, by adjusting $g_{e \mu}=g_{e \tau}=0$ and $g_{e e}=g_{\mu \mu}$. The Majorana phase $\alpha_{2}$ defined in the convention by Particle Data Group [38] is $\alpha_{2}=\arg \left(m_{2} / m_{3}\right)$ here. We choose $f_{0}^{2}=0.002$, $m_{k}=1.2 \mathrm{TeV}$, and $M_{0} /\left(48 \pi^{2}\right)=500 \mathrm{GeV}$. In Fig. 8 (right), we show $\operatorname{Br}(\tau \rightarrow 3 \mu)$. Because $g_{e e}=g_{\mu \mu}$ is chosen, we obtain $\operatorname{Br}\left(\tau^{-} \rightarrow \mu^{+} e^{-} e^{-}\right)=\operatorname{Br}(\tau \rightarrow 3 \mu)$. As can be seen in Fig. 8 (left), $\left|G_{2}\right| / G_{F}$ can be as large as the current experimental bound shown in Eq. (2.32).

We comment on the model-parameter dependence of the Mu-to- $\overline{\mathrm{Mu}}$ transition. The coefficient of the transition operator is roughly proportional to the model parameters as

$$
\frac{\left|G_{2}\right|}{\sqrt{2}}=\frac{\left|g_{e e} g_{\mu \mu}\right|}{8 m_{k}^{2}} \propto \frac{1}{f_{0}^{4}} \frac{\max \left(m_{k}^{4}, m_{h}^{4}\right)}{\mu_{h h k}^{2} m_{k}^{2}} \frac{g_{e e}}{g_{\mu \mu}} .
$$

Here we use Eq. (7.15) to include the constraint to reproduce the neutrino mass. The nonobservation of $\mu \rightarrow$ er gives the lower bound of $m_{h}^{4} / f_{0}^{4}$. If $f_{0}$ (namely $f$ ) becomes smaller, the coupling $g_{\mu \mu}$ needs to be larger to reproduce the size of neutrino mass $m_{3}$, and thus, the Mu-to- $\overline{\mathrm{Mu}}$ transition becomes larger. The scalar trilinear coupling $\mu_{h h k}$ should not be much larger than $m_{h}$ and $m_{k}$ to avoid a charge breaking global minimum. Therefore, the search of the Mu-to- $\overline{\mathrm{Mu}}$ transition gives a good test of the Zee-Babu model in the range of $g_{e e} \sim g_{\mu \mu}$.

Since $g_{\mu \tau}$ cannot be eliminated, the $\tau \rightarrow 3 \mu$ and $\tau^{-} \rightarrow$ $\mu^{+} e^{-} e^{-}$processes are generated:

$$
\begin{aligned}
& \left\{\operatorname{Br}(\tau \rightarrow 3 \mu), \operatorname{Br}\left(\tau^{-} \rightarrow \mu^{+} e^{-} e^{-}\right)\right\} \\
& \quad=8\left|\frac{G_{2}}{G_{F}}\right|^{2} \operatorname{Br}(\tau \rightarrow \mu \nu \bar{\nu}) \times\left\{\left|\frac{g_{\mu \tau}}{g_{e e}}\right|^{2},\left|\frac{g_{\mu \tau}}{g_{\mu \mu}}\right|^{2}\right\} .
\end{aligned}
$$



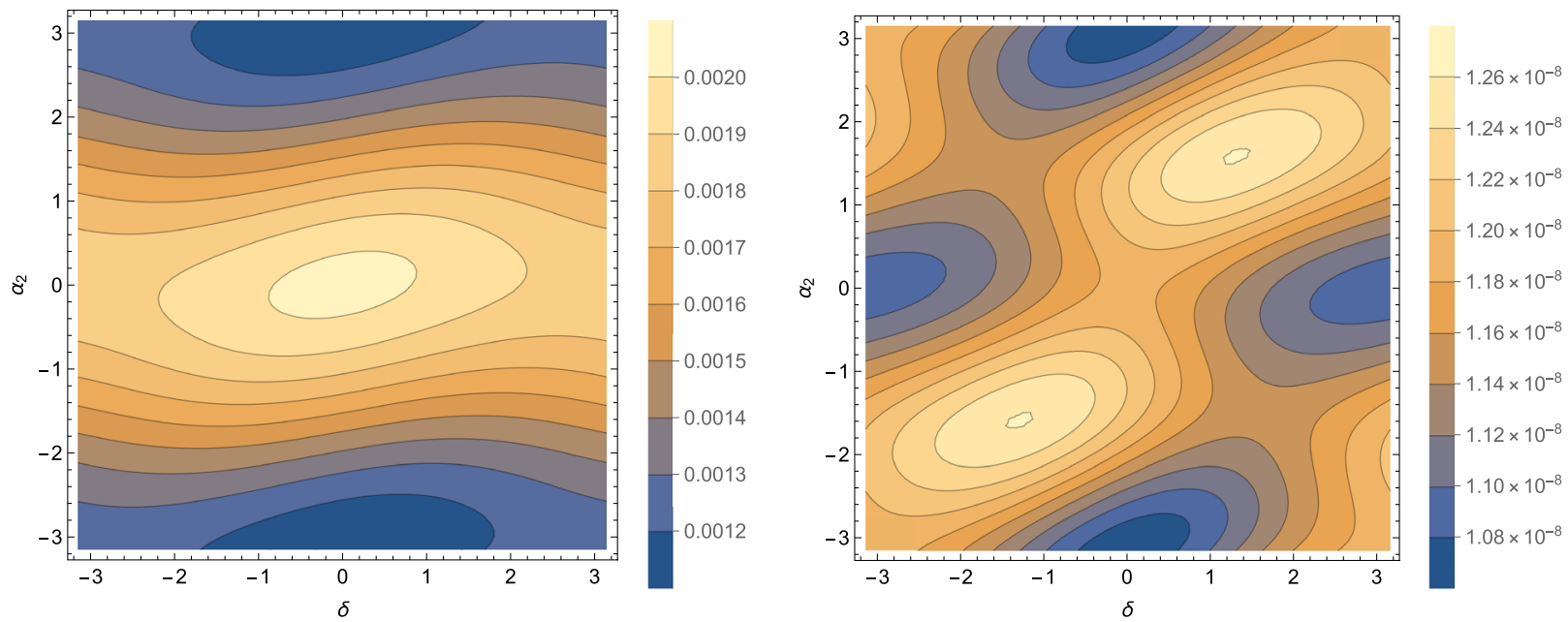

FIG. 8. The contour plots of $\left|G_{2}\right| / G_{F}$ (left), $\operatorname{Br}(\tau \rightarrow 3 \mu)$ (right) as functions of the Dirac phase $\delta$ and the Majorana phase $\alpha_{2}$. The choices of parameters in the Zee-Babu model are given in the text.

Because of $g_{\mu \tau} \simeq g_{\mu \mu} m_{\mu} / m_{\tau}$, if the Mu-to- $\overline{\mathrm{Mu}}$ transition is observed at $G_{2} / G_{F} \sim 10^{-3}$, those two LFV tau decays will be observed.

We note on the case of inverted mass hierarchy. Similarly, the rank-2 neutrino mass matrix $\left(m_{3}=0\right.$ in this case) is given as

$$
M_{\nu}=m_{1} u_{1}^{*} u_{1}^{\dagger}+m_{2} u_{2}^{*} u_{2}^{\dagger},
$$

and the solution of Eq. (7.2) is

$$
f=f_{0}\left(\begin{array}{ccc}
0 & U_{\tau 3} & -U_{\mu 3} \\
-U_{\tau 3} & 0 & U_{e 3} \\
U_{\mu 3} & -U_{e 3} & 0
\end{array}\right),
$$

and

$$
\begin{aligned}
\frac{f_{0}^{2}}{M_{0}} M_{e} g M_{e}= & m_{1} u_{2} u_{2}^{T}+m_{2} u_{1} u_{1}^{T}+a_{1}\left(u_{3} u_{1}^{T}+u_{1} u_{3}^{T}\right) \\
& +a_{2}\left(u_{3} u_{2}^{T}+u_{2} u_{3}^{T}\right)+a_{3} u_{3} u_{3}^{T} .
\end{aligned}
$$

The size of $g_{\mu \mu}$ becomes larger than the one in the normal hierarchy to make $e e, e \mu$, and $e \tau$ elements of $M_{e} g M_{e}$ to be small, under the same model parameters above. This is because $U_{e 3}$ is small compared to the other elements, and $a_{3}$ needs to be larger. Consequently, the coefficient $G_{2}$ becomes larger than the current bound unless $g_{e e}$ is made to be much smaller than $g_{\mu \mu}$.

\section{Cocktail model}

In the cocktail model [57], an inert Higgs doublet $\eta$ (which does not acquire a vev) and a hypercharge $Y=1$ $S U(2)_{L}$ singlet $S^{+}$are introduced in addition to the doubly charged scalar $k^{++}$. Contrary to the Zee-Babu model, the
$S^{+}$scalar does not couple to leptons directly, and the $\mu \rightarrow e \gamma$ induced by $S^{+}$loop does not bother us. The neutrino masses are generated by three-loop diagrams, which look like a cocktail glass as shown in Fig. 9. The mass matrix is given as

$$
\left(M_{\nu}\right)_{\alpha \beta}=\frac{1}{\left(16 \pi^{2}\right)^{3}} m_{\alpha} g_{\alpha \beta} m_{\beta} \frac{F_{\text {cocktail }}}{m_{k}},
$$

where $g$ is a doubly charged scalar coupling to right-handed charged leptons (same as in Zee-Babu model), $m_{\alpha}(\alpha=e$, $\mu, \tau)$ is the charged lepton mass, $m_{k}$ is a doubly charged scalar mass, and $F_{\text {cocktail }}$ stands for a loop function containing couplings in the model.

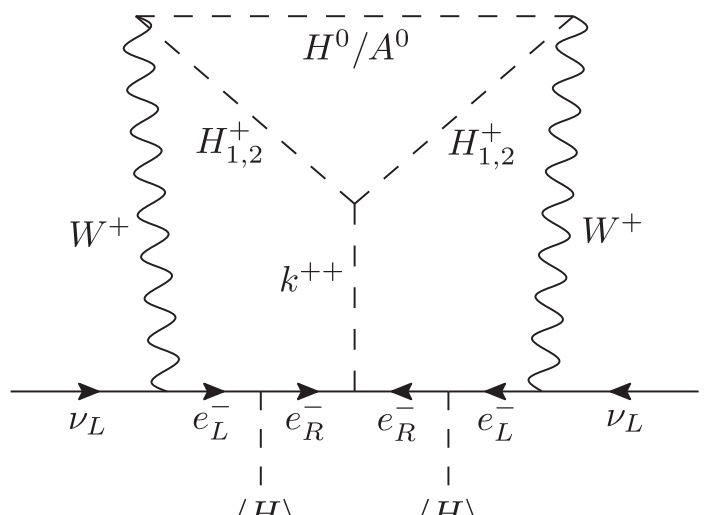

FIG. 9. A diagram to induce the neutrino mass in the cocktail model. The charged scalar in the inert doublet $\eta$ and the $S U(2)_{L}$ singlet scalar $S^{+}$are mixed to be $H_{1,2}^{+}$. By splitting the masses of the real and imaginary parts $\left(H^{0}, A^{0}\right)$ of the neutral scalar in $\eta$, neutrino masses are generated. The symbol $\langle H\rangle$ stands for the vev of the SM Higgs boson. 
The mass scale to generate the neutrino mass matrix, $M_{\nu}=U^{*} \operatorname{diag}\left(m_{1}, m_{2}, m_{3}\right) U^{\dagger}$, in the normal mass ordering is estimated as

$$
\frac{m_{k}}{F_{\text {cocktail }}} \sim \frac{1}{\left(16 \pi^{2}\right)^{3}} \frac{m_{\mu}^{2} g_{\mu \mu}}{m_{3} U_{\mu 3}^{2}} \sim 100 \mathrm{GeV} \times g_{\mu \mu}
$$

A large value of $F_{\text {cocktail }}$ is needed for a realistic model to satisfy the experimental constraints. More numerical works to obtain the scale by model parameters can be found in [58]. One can immediately notice that the magnitude of $e e$ and $e \mu$ elements of the neutrino mass matrix $M_{\nu}$ should be much smaller than the magnitude of $\mu \mu$ element unless $g_{e e}$ and $g_{e \mu}$ are much larger than 1. Indeed, we want to make $g_{e \mu} \rightarrow 0$ to obtain a reachable $\mathrm{Mu}$-to- $\overline{\mathrm{Mu}}$ transition while suppressing the $\mu \rightarrow 3 e$ process. If the $e e$ and $e \mu$ elements of $M_{\nu}$ are much smaller than the $\mu \mu$ element, neutrino mixings and mass ratio, and phases are constrained. The analytic relation of the neutrino mixings and mass ratio is given in Ref. [62]. In Fig. 10, we show the relation between the PMNS phase $\delta$ and $\theta_{23}$. We vary $\theta_{12}$ since the relation is sensitive to it. The $3 \sigma$ range of $\theta_{12}$ is $31.3^{\circ}-35.9^{\circ}$ by NuFIT 5.0 [63]. Because $\theta_{23}$ is in the $3 \sigma$ range of $40^{\circ}-52^{\circ}$, it predicts that $\delta$ is preferred to be in the second or third quadrant roughly.

Let us choose $\theta_{12}=33.4^{\circ}, \theta_{13}=8.57^{\circ}, \Delta m_{\text {sol }}^{2}=$ $7.42 \times 10^{-5} \mathrm{eV}^{2}$, and $\Delta m_{\mathrm{atm}}^{2}=2.52 \times 10^{-3} \mathrm{eV}^{2}$. Then, we can choose $\theta_{23}=45^{\circ}$ and $\delta \simeq \pi$ as a benchmark point. For $g_{e e}=g_{\mu \mu}$ and $g_{e \mu}=0$, we obtain

$$
g_{\alpha \beta}=\left(\begin{array}{ccc}
1 & 0 & -4.57 \\
0 & 1 & 0.0454 \\
-4.57 & 0.0454 & 0.00321
\end{array}\right) g_{\mu \mu} .
$$

Because of $m_{e} \ll m_{\mu}$, the numerical values of the elements are insensitive to $g_{e e}$ to reproduce the neutrino mass matrix.

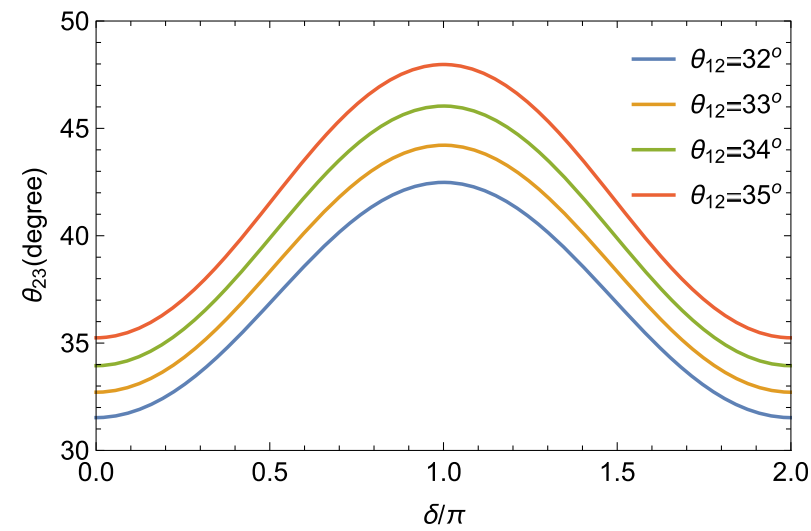

FIG. 10. The relation between $\delta$ (radian) and $\theta_{23}$ for various $\theta_{12}$ in the case of $\left(M_{\nu}\right)_{e e, e \mu} \rightarrow 0$. We use central values for the 1-3 neutrino mixing and mass squared differences: $\theta_{13}=8.57^{\circ}$, $\Delta m_{\mathrm{sol}}^{2}=7.42 \times 10^{-5} \mathrm{eV}^{2}$, and $\Delta m_{\mathrm{atm}}^{2}=2.52 \times 10^{-3} \mathrm{eV}^{2}[63]$.
It is important to notice that $g_{e \tau}$ is large, and the Mu-to- $\overline{\mathrm{Mu}}$ transition is bounded by the $\tau \rightarrow 3 e$ process:

$$
\frac{\left|G_{2}\right|}{G_{F}}=\frac{\operatorname{Br}(\tau \rightarrow 3 e)}{\operatorname{Br}(\tau \rightarrow e \nu \bar{\nu})} \frac{1}{2 \sqrt{2}}\left|\frac{g_{\mu \mu}}{g_{e \tau}}\right|<3 \times 10^{-5} .
$$

The $\mu \rightarrow e \gamma$ process also bounds the Mu-to- $\overline{\mathrm{Mu}}$ transition similarly to Eq. (5.26) as

$$
\frac{\left|G_{2}\right|}{G_{F}}<3.8 \times 10^{-6}\left|\frac{g_{e e} g_{\mu \mu}}{g_{e \tau} g_{\mu \tau}}\right|=1.8 \times 10^{-5} \times\left|\frac{g_{e e}}{g_{\mu \mu}}\right|
$$

in this benchmark point. We note that the Zee-Babu model has freedom to suppress $g_{e \tau}$, while in the cocktail model, $g_{e \tau}$ is needed to generate $\theta_{12}$ and $\theta_{13}$ neutrino mixings. Consequently, the Mu-to- $\overline{\mathrm{Mu}}$ transition is bounded in the cocktail model rather than the Zee-Babu model.

\section{B. Charged Higgs contribution}

The Dirac mass is supposed to be forbidden by $Z_{2}$ symmetry. Namely, the Dirac Yukawa coupling with the SM Higgs doublet $\Phi$ is absent, but couplings with an additional inert doublet $\eta$ are allowed:

$$
-\mathcal{L} \supset y_{\alpha i} \overline{\ell_{\alpha}} P_{R} N_{i} \eta+\frac{1}{2} M_{i} \overline{N_{i}^{c}} N_{i}+\text { H.c. }
$$

If the scalar potential contains the $\lambda_{5}$ term,

$$
V \supset \frac{\lambda_{5}}{4}\left(\eta \Phi^{\dagger}\right)^{2}+\text { H.c. }
$$

the masses $\left(m_{H}\right.$ and $\left.m_{A}\right)$ of the real and imaginary parts $\left(H^{0}\right.$ and $A^{0}$ ) of the neutral Higgs boson in the inert doublet $\eta$ are split:

$$
m_{H}^{2}-m_{A}^{2}=\lambda_{5} v^{2},
$$

where $v$ is the vev of the SM Higgs boson. Then, the active neutrino masses are generated radiatively by the diagram given in Fig. 11 as [59]

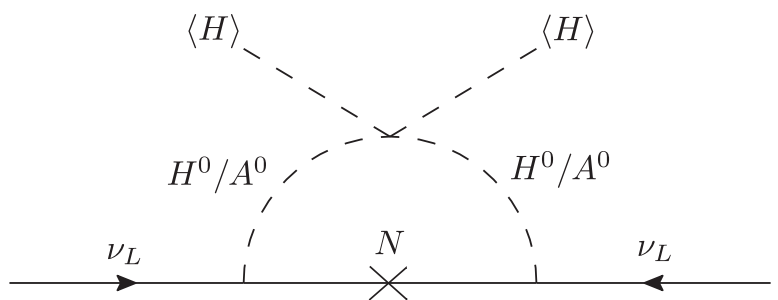

FIG. 11. The diagram to generate the neutrino mass radiatively in the model without the Dirac neutrino masses. The symbol $\langle H\rangle$ stands for the vev of the SM Higgs boson. 


$$
\begin{aligned}
\left(M_{\nu}\right)_{\alpha \beta} & =\frac{1}{16 \pi^{2}} y_{\alpha i} y_{\beta i} M_{i}\left(\frac{m_{H}^{2}}{m_{H}^{2}-M_{i}^{2}} \ln \frac{m_{H}^{2}}{M_{i}^{2}}-\frac{m_{A}^{2}}{m_{A}^{2}-M_{i}^{2}} \ln \frac{m_{A}^{2}}{M_{i}^{2}}\right) \\
& \simeq \frac{\lambda_{5} v^{2}}{16 \pi^{2}} \frac{y_{\alpha i} y_{\beta i}}{M_{i}}\left(\frac{M_{i}^{2}}{m_{H}^{2}-M_{i}^{2}}-\frac{M_{i}^{4}}{\left(m_{H}^{2}-M_{i}^{2}\right)^{2}} \ln \frac{m_{H}^{2}}{M_{i}^{2}}\right) .
\end{aligned}
$$

The charged Higgs boson in $\eta$ can generate the Mu-to-Mu transition by the box diagrams in Fig. 12:

$$
\begin{aligned}
\frac{G_{1}}{\sqrt{2}}= & \frac{y_{e i}^{*} y_{\mu i} y_{e j}^{*} y_{\mu j}}{512 \pi^{2} m_{\eta}^{2}} I_{2}\left(x_{i}, x_{j}\right) \\
& +\frac{\left(y_{e i}^{*}\right)^{2}\left(y_{\mu j}\right)^{2}}{256 \pi^{2} m_{\eta}^{2}} \sqrt{x_{i} x_{j}} I_{1}\left(x_{i}, x_{j}\right),
\end{aligned}
$$

where $x_{i}=M_{i}^{2} / m_{\eta}^{2}$, and $I_{n}\left(x_{i}, x_{j}\right)$ 's are the box loop functions given in Appendix D, and $m_{\eta}$ stands for the charged Higgs boson mass. The first term is bounded by $\mu \rightarrow e \gamma$. Let us consider whether the Mu-to- $\overline{\mathrm{Mu}}$ transition from the second term can be generated avoiding the $\mu \rightarrow e \gamma$ constraint. To do that, we consider

$$
y_{\alpha i}=\left(\begin{array}{ccc}
y_{e 1} & 0 & 0 \\
0 & y_{\mu 2} & 0 \\
y_{\tau 1} & y_{\tau 2} & y_{\tau 3}
\end{array}\right)
$$

to eliminate the one-loop $\mu \rightarrow e \gamma$ amplitude via $\eta$ loop. (For the purpose to eliminate $\mu \rightarrow e \gamma$, one of $h_{e 3}$ and $h_{\mu 3}$ can be nonzero. We here suppose that both are zero to reduce the number of parameters.) Then the neutrino mass matrix is

$$
M_{\nu}=\frac{\lambda_{5} v^{2}}{16 \pi^{2}}\left(\begin{array}{ccc}
\frac{y_{e 1}^{2}}{\tilde{M}_{1}} & 0 & \frac{y_{e 1} y_{\tau 1}}{\bar{M}_{1}} \\
0 & \frac{y_{\mu_{2}}^{2}}{\tilde{M}_{2}} & \frac{y_{\mu 2} y_{\tau 2}}{\tilde{M}_{2}} \\
\frac{y_{e 1} y_{\tau 1}}{\tilde{M}_{1}} & \frac{y_{\mu 2} y_{\tau 2}}{\tilde{M}_{2}} & \frac{y_{\tau 1}^{2}}{\tilde{M}_{1}}+\frac{y_{\tau 2}^{2}}{\tilde{M}_{2}}+\frac{y_{\tau 3}^{2}}{\tilde{M}_{3}}
\end{array}\right) \text {, }
$$

where $\tilde{M}_{i}$ is defined to be $M_{i}$ divided by a loop function so that the neutrino mass matrix is $\propto y_{\alpha i} y_{\beta i} / \hat{M}_{i}$. The size of the coupling is estimated as

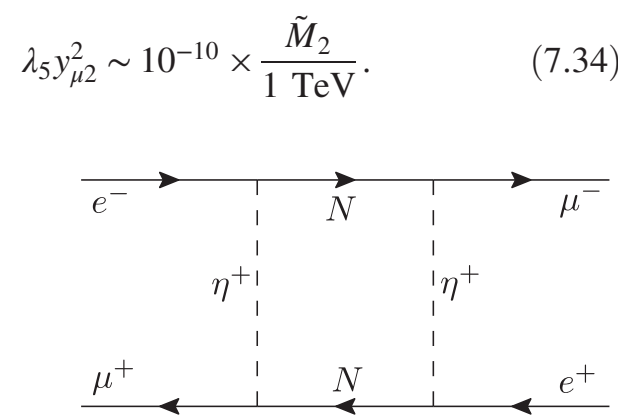

Because there are four complex parameters (up to normalization), one can fit three neutrino mixings, one mass ratio, and phases, in principle. The lightest neutrino mass $m_{1}$ (with its phase) is a function of the other parameters since the $e \mu$ element is chosen to be zero.

Though the couplings have been chosen to eliminate the $\mu \rightarrow e \gamma$ process, one needs to care about $\tau \rightarrow l_{\alpha} \gamma\left(l_{\alpha}=e, \mu\right)$ processes since $y_{\tau 1}$ and $y_{\tau 2}$ are needed to reproduce the neutrino mixings:

$\operatorname{Br}\left(\tau \rightarrow l_{\alpha} \gamma\right)=\frac{3 \alpha}{16 \pi}\left|\frac{y_{\alpha i} y_{\tau i}^{*} F_{N}\left(x_{i}\right)}{G_{F} m_{\eta}^{2}}\right|^{2} \operatorname{Br}\left(\tau \rightarrow l_{\alpha} \nu \bar{\nu}\right)$,

where

$$
F_{N}(x)=\frac{1-6 x+3 x^{2}+2 x^{3}-6 x^{2} \ln x}{12(1-x)^{4}}
$$

We also need to care about muon $g-2$ since the loop contribution gives a negative contribution to it:

$$
\Delta a_{\mu}=-\frac{1}{8 \pi^{2}} \frac{m_{\mu}^{2}}{m_{\eta}^{2}}\left|y_{\mu i}\right|^{2} F_{N}\left(x_{i}\right) .
$$

We can check that the experimental bound can be satisfied even if the nonzero elements of $y_{\alpha i}$ are $O(1)$ for $m_{\eta}=500 \mathrm{GeV}$. The transition amplitude can be maximal for $M_{i} \simeq m_{\eta}$, and we find $\left|G_{1}\right| / G_{F} \lesssim O\left(10^{-5}\right)$ for $m_{\eta}=500 \mathrm{GeV}$ and $\left|y_{\alpha i}\right|<1$.

\section{C. $N S^{+} e_{R}$ coupling}

We consider models with right-handed charged lepton couplings to SM singlet fermion $N$ :

$$
-\mathcal{L}=h_{\alpha i} \overline{e_{\alpha}^{c}} P_{R} N_{i} S^{+}+\frac{1}{2} M_{i} \overline{N_{i}^{c}} N_{i}+M_{S}^{2} S^{+} S^{-},
$$

where $S^{+}$is a $S U(2)_{L}$ singlet with hypercharge $Y=1$. The transition operator $Q_{2}$ is generated and the coefficient is

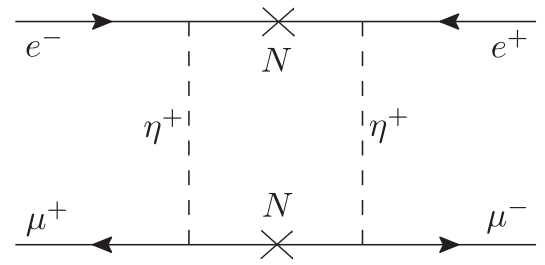

FIG. 12. The box loop diagrams to generate the Mu-to- $\overline{\mathrm{Mu}}$ transition via the charged Higgs bosons. 


$$
\begin{aligned}
\frac{G_{2}}{\sqrt{2}}= & \frac{h_{e i} h_{\mu i}^{*} h_{e j} h_{\mu j}^{*}}{512 \pi^{2} M_{S}^{2}} I_{2}\left(x_{i}, x_{j}\right) \\
& +\frac{\left(h_{e i}\right)^{2}\left(h_{\mu j}^{*}\right)^{2}}{256 \pi^{2} M_{S}^{2}} \sqrt{x_{i} x_{j}} I_{1}\left(x_{i}, x_{j}\right),
\end{aligned}
$$

where $x_{i}=M_{i}^{2} / M_{S}^{2}$.

\section{KNT model}

In the model by Krauss-Nasri-Trodden (KNT) [60], two $Y=1 S U(2)_{L}$ singlet scalars (we call them $S_{1}$ and $S_{2}$ ) are introduced:

$$
\begin{aligned}
-\mathcal{L} \supset & f_{\alpha \beta} \overline{\ell_{\alpha}^{c}} \cdot \ell_{\beta} S_{1}^{+}+h_{\alpha j} \overline{e_{\alpha}^{c}} P_{R} N_{j} S_{2}^{+} \\
& +\lambda_{S}\left(S_{1}^{+} S_{2}^{-}\right)^{2}+\text { H.c. }
\end{aligned}
$$

The neutrino mass is generated by a three-loop diagram shown in Fig. 13:

$$
\begin{aligned}
\left(M_{\nu}\right)_{\alpha \beta}= & \frac{\lambda_{S}}{\left(16 \pi^{2}\right)^{3} M_{S_{2}}^{2}} f_{\alpha \alpha^{\prime}} m_{\alpha^{\prime}} h_{\alpha^{\prime} i} \\
& \times F_{\mathrm{KNT}}\left(M_{N i} / M_{S_{2}}, M_{S_{1}} / M_{S_{2}}\right) h_{\beta^{\prime} i} m_{\beta^{\prime}} f_{\beta \beta^{\prime}},
\end{aligned}
$$

where $m_{\alpha}=\left(m_{e}, m_{\mu}, m_{\tau}\right)$ and $F_{\mathrm{KNT}}$ is a loop function. The equation can be solved just similarly to the Zee-Babu model. However, the coupling $h$ becomes large to be in a nonperturbative region if one assumes that the observed neutrino masses are all covered by this contribution. Actually, in the Zee-Babu model in which the neutrino masses are generated by the two-loop diagram, the coupling can be $O(1)$ for several hundred GeV scalar masses, and thus, one can imagine that the coupling needs to be large to generate the neutrino mass by three-loop. This is because the antisymmetric coupling $f$ needs to be small to avoid $\mu \rightarrow e \gamma$ constraint. Of course, it is possible to give up on explaining the entire neutrino mass matrix with this loop-induced contribution and assume that the neutrino masses come primarily from somewhere else. In such a situation, the $h$ couplings can be $O(1)$ without a contradiction with observables, and they can induce the

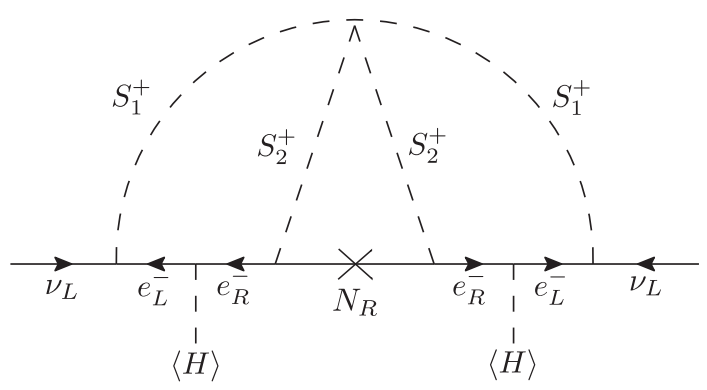

FIG. 13. A diagram to induce the neutrino mass in the KNT model. The symbol $\langle H\rangle$ stands for the vev of the SM Higgs boson.
Mu-to- $\overline{\mathrm{Mu}}$ transition, potentially as large as the current bound, from the second term in Eq. (7.39).

\section{AKS model}

In the model by Aoki-Kanemura-Seto (AKS) [61], two Higgs doublets $\Phi_{1}$ and $\Phi_{2}$ to have a physical charged Higgs scalar $(H)$ in the loop, and one real scalar singlet $\eta^{0}$ are introduced. The neutrino mass is generated by three-loop diagrams such as shown in Fig. 14:

$$
\left(M_{\nu}\right)_{\alpha \beta}=\frac{\kappa^{2} \tan ^{2} \beta}{\left(16 \pi^{2}\right)^{3}} m_{\alpha} m_{\beta} h_{\alpha i} h_{\beta i} \frac{F_{\mathrm{AKS}}\left(M_{N i}, M_{\eta}, M_{S}, M_{H^{+}}\right)}{M_{N i}},
$$

where $\kappa$ is $\Phi_{1} \Phi_{2} \eta S$ coupling.

Let us consider if the observed parameters of neutrino oscillations can be reproduced with satisfying the $\mu \rightarrow e \gamma$ constraint, and see if the Mu-to- $\overline{\mathrm{Mu}}$ transition can be induced by the $h_{\alpha i}$ coupling. To do that, let us consider

$$
h_{\alpha i}=\left(\begin{array}{ccc}
h_{e 1} & 0 & 0 \\
0 & h_{\mu 2} & 0 \\
h_{\tau 1} & h_{\tau 2} & h_{\tau 3}
\end{array}\right) \text {, }
$$

similarly to Eq. (7.32), in order to eliminate the one-loop $\mu \rightarrow e \gamma$ amplitude via $h_{\alpha i}$ coupling. Then the neutrino mass matrix is

$M_{\nu}=\frac{\kappa^{2} \tan ^{2} \beta}{\left(16 \pi^{2}\right)^{3}}\left(\begin{array}{ccc}\frac{m_{e}^{2} h_{e 1}^{2}}{\tilde{M}_{1}} & 0 & \frac{m_{e} m_{\tau} h_{e 1} h_{\tau 1}}{\tilde{M}_{1}} \\ 0 & \frac{m_{\mu}^{2} h_{\mu 2}^{2}}{\tilde{M}_{2}} & \frac{m_{\mu} m_{\tau} h_{\mu 2} h_{\tau 2}}{\tilde{M}_{2}} \\ \frac{m_{e} m_{\tau} h_{e 1} h_{\tau 1}}{\tilde{M}_{1}} & \frac{m_{\mu} m_{\tau} h_{\mu 2} h_{\tau 2}}{\tilde{M}_{2}} & m_{\tau}^{2}\left(\frac{h_{\tau 1}^{2}}{\tilde{M}_{1}}+\frac{h_{\tau 2}^{2}}{\tilde{M}_{2}}+\frac{h_{\tau 3}^{2}}{\tilde{M}_{3}}\right)\end{array}\right)$,

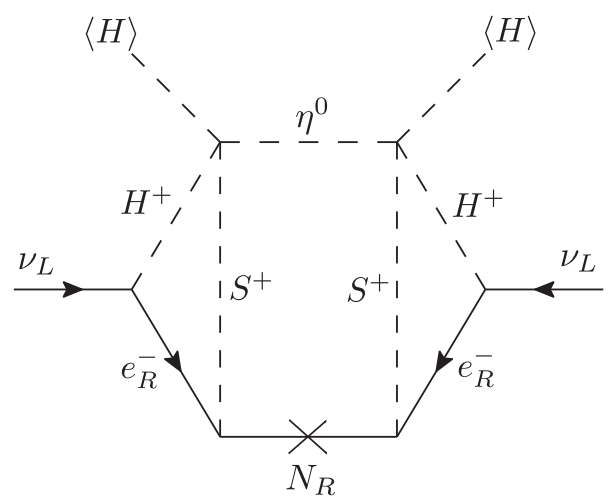

FIG. 14. A diagram to induce the neutrino mass in the AKS model. The symbol $\langle H\rangle$ stands for the vev of the SM Higgs boson. 
where $\tilde{M}_{i}=M_{N i} / F_{\mathrm{AKS}}\left(M_{N i}\right)$. We obtain the mass scale of $\tilde{M}_{2}$ as

$$
\tilde{M}_{2} \simeq 100 \mathrm{GeV} \times h_{\mu 2}^{2} \kappa^{2} \tan ^{2} \beta
$$

There are two types of solutions:

(1) Naive solution: $h_{\mu 2} \ll h_{e 1} \sim 1$.

If there is no cancellation in the $\tau \tau$ element of $M_{\nu}$, one needs $h_{\mu 2} \ll h_{e 1}$ to realize the neutrino mixings. This is due to $m_{e} \ll m_{\mu}$. Since $h_{\mu 2}$ is small, a large value of $\kappa \tan \beta$ is needed to obtain the proper size of the neutrino mass in this solution. Any observed neutrino mixings (within errors) and a PMNS phase $\delta$ can be realized. The Mu-to- $\overline{\mathrm{Mu}}$ transition is estimated as $\left|G_{2}\right| / G_{F} \lesssim O\left(10^{-7}\right)$. (If one allows a nearly nonperturbative value of $h_{e 1} \sim 10$, the Mu-to- $\overline{\mathrm{Mu}}$ transition can be enlarged, though.)

(2) $h_{e 1} \sim h_{\mu 2}$.

If we allow a tuning of the $\tau \tau$ element of $M_{\nu}$ $\left(h_{\tau 1}^{2} / \tilde{M}_{1}+h_{\tau 3}^{2} / \tilde{M}_{3} \ll h_{\tau 2}^{2} / \tilde{M}_{2}\right)$ to obtain the atmospheric mixing properly, $h_{e 1} \sim h_{\mu 2}$ can be allowed. Both $e e$ and $e \mu$ elements of $M_{\nu}$ are much smaller than $\mu \mu$ element in this solution, and therefore, the neutrino mixings and the Dirac phase $\delta$ are related as shown in Fig. 10. To realize the solar neutrino mixing, one needs to enlarge $h_{\tau 1}$ compared to the naive solution. Since $h_{\tau 1}$ becomes $O(1)$, one needs to care about $\tau \rightarrow e \gamma$ process. Our estimation of the Mu-to- $\overline{\mathrm{Mu}}$ transition is $\left|G_{2}\right| / G_{F} \lesssim O\left(10^{-6}\right)$.

\section{NEUTRAL SCALAR EXCHANGE}

The Mu-to- $\overline{\mathrm{Mu}}$ transition induced by the neutral scalar exchange shown in Fig. 15 is considered [64].

In general two-Higgs-doublets model, so-called type-III, the Yukawa couplings can be written as

$$
-\mathcal{L}=\left(Y_{1}\right)_{i j} \overline{\ell_{i L}} e_{j R} \Phi_{1}^{\prime}+\left(Y_{2}\right)_{i j} \overline{\ell_{i L}} e_{j R} \Phi_{2}^{\prime}+\text { H.c. }
$$

and the vevs of the neutral components are $\left\langle\Phi_{1}^{\prime 0}\right\rangle=$ $v \cos \beta^{\prime},\left\langle\Phi_{2}^{\prime 0}\right\rangle=v \sin \beta^{\prime}$. Redefining the Higgs fields so that $\Phi_{1}$ does not acquire a vev,

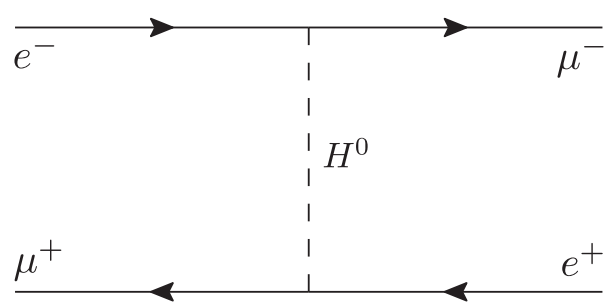

FIG. 15. The tree-level exchange of a neutral scalar boson $\left(H^{0}\right.$, here) to induce the $\mathrm{Mu}-\mathrm{to}-\overline{\mathrm{Mu}}$ transition.

$$
\left(\begin{array}{l}
\Phi_{2} \\
\Phi_{1}
\end{array}\right)=\left(\begin{array}{cc}
\sin \beta^{\prime} & \cos \beta^{\prime} \\
-\cos \beta^{\prime} & \sin \beta^{\prime}
\end{array}\right)\left(\begin{array}{l}
\Phi_{2}^{\prime} \\
\Phi_{1}^{\prime}
\end{array}\right)
$$

we can rewrite the Yukawa interaction as

$$
-\mathcal{L}=\left(Y_{e}\right)_{i j} \overline{\ell_{i L}} e_{j R} \Phi_{2}+\rho_{i j} \bar{\ell}_{i L} e_{j R} \Phi_{1}+\text { H.c. }
$$

where

$$
\begin{aligned}
Y_{e} & =Y_{1} \sin \beta^{\prime}+Y_{2} \cos \beta^{\prime}, \\
\rho & =-Y_{1} \cos \beta^{\prime}+Y_{2} \sin \beta^{\prime} .
\end{aligned}
$$

We can redefine $\sin \beta=1$. The Yukawa coupling $Y_{e}$ generates the charged lepton masses, and thus, we work on the flavor basis where $Y_{e}$ is diagonal, $Y_{e}=$ $\operatorname{diag}\left(y_{e}, y_{\mu}, y_{\tau}\right)$. The neutral physical Higgs interaction can be written as

$$
\begin{aligned}
-\mathcal{L}= & \frac{1}{\sqrt{2}}\left(Y_{e} c_{\alpha}+\rho s_{\alpha}\right)_{i j} \overline{e_{i L}} e_{j R} h \\
& +\frac{1}{\sqrt{2}}\left(Y_{e} s_{\alpha}-\rho c_{\alpha}\right)_{i j} \overline{e_{i L}} e_{j R} H \\
& +i \frac{1}{\sqrt{2}} \rho_{i j} \overline{e_{i L}} e_{j R} A+\text { H.c. },
\end{aligned}
$$

where $s_{\alpha}=\sin \alpha$ and $c_{\alpha}=\cos \alpha$ are the mixings of $C P$ even Higgs bosons $(h$ and $H$ ). Integrating out the neutral Higgs bosons, $h, H$, and $A$, we extract the terms which can be the transition operators:

$$
\begin{aligned}
-\mathcal{L}= & -\frac{1}{4}\left(\rho_{21}^{2}\left(\overline{\mu_{L}} e_{R}\right)^{2}+\rho_{12}^{* 2}\left(\overline{\mu_{R}} e_{L}\right)^{2}\right)\left(\frac{s_{\alpha}^{2}}{m_{h}^{2}}+\frac{c_{\alpha}^{2}}{m_{H}^{2}}-\frac{1}{m_{A}^{2}}\right) \\
& -\frac{1}{2} \rho_{21} \rho_{12}^{*}\left(\overline{\mu_{L}} e_{R}\right)\left(\overline{\mu_{R}} e_{L}\right)\left(\frac{s_{\alpha}^{2}}{m_{h}^{2}}+\frac{c_{\alpha}^{2}}{m_{H}^{2}}+\frac{1}{m_{A}^{2}}\right) \\
& + \text { H.c., }
\end{aligned}
$$

and

$$
\begin{aligned}
& \frac{G_{3}}{\sqrt{2}}=\frac{1}{16} \rho_{21} \rho_{12}^{*}\left(\frac{s_{\alpha}^{2}}{m_{h}^{2}}+\frac{c_{\alpha}^{2}}{m_{H}^{2}}+\frac{1}{m_{A}^{2}}\right), \\
& \frac{G_{4}}{\sqrt{2}}=-\frac{1}{16} \rho_{12}^{* 2}\left(\frac{s_{\alpha}^{2}}{m_{h}^{2}}+\frac{c_{\alpha}^{2}}{m_{H}^{2}}-\frac{1}{m_{A}^{2}}\right), \\
& \frac{G_{5}}{\sqrt{2}}=-\frac{1}{16} \rho_{21}^{2}\left(\frac{s_{\alpha}^{2}}{m_{h}^{2}}+\frac{c_{\alpha}^{2}}{m_{H}^{2}}-\frac{1}{m_{A}^{2}}\right) .
\end{aligned}
$$

If we suppose that $\rho_{21}$ and $\rho_{12}$ are not zero with the other $\rho_{i j}=0$ and $\sin \alpha(=\cos (\beta-\alpha)) \rightarrow 0$ (alignment limit), LFV processes such as $\mu \rightarrow e \gamma$ and $\mu \rightarrow 3 e$ do not occur. In the limit, one obtains $G_{4}, G_{5} \rightarrow 0$. Actually, in that case, 
there is a global discrete $Z_{4}$ symmetry, and the charge assignments are

$$
e_{L}: 1, \quad e_{R}: 1, \quad \mu_{L}: 3, \quad \mu_{R}: 3, \quad \Phi_{1}: 2,
$$

and the charges of the others are 0 . Therefore, once the selection of $\rho$ with the alignment limit is given, LFV is not generated perturbatively. We note that the electron mass can have a loop correction from $\rho_{12} \rho_{21}$. The $\rho$ terms can also induce the electron $g-2$ and electric dipole moment (therefore, $\rho_{12} \rho_{21}$ should be real). The coefficient $G_{3}$ can be generated satisfying the LFV constraints in the alignment limit, and the Mu-to-Mu transition can be around the current experimental bound.

\section{SUSY}

Similarly to the meson mixings, the box diagram in which superparticles propagate can generate the transition operators. Indeed, the minimally extended SUSY standard model (MSSM) contains Majorana particles known as gauginos: bino $\tilde{B}$ and wino $\tilde{W}^{0}$. Though the Majorana property of gauginos can be utilized to generate the $\mathrm{Mu}-\mathrm{to}-\overline{\mathrm{Mu}}$ transition, the transition probability is bounded by the $\mu \rightarrow e \gamma$ constraint as we have seen in various models.

In the MSSM, the left-handed slepton doublet and the down-type Higgs doublet have the same quantum numbers, and thus, so-called $R$-parity is introduced to distinguish them. The $R$-parity is also needed to avoid rapid proton decays. If the $R$-parity is broken in the lepton sector, the Mu-to- $\overline{\mathrm{Mu}}$ transition can be induced at the tree-level sneutrino exchange [65], as a simple corollary of the neutral Higgs exchange (in the alignment limit) in the previous section.

In this section, we first briefly describe the previously well-known $\mathrm{Mu}$-to- $\overline{\mathrm{Mu}}$ transition in the $R$-parity violating SUSY model, and then study the box contribution of the neutralinos and charginos in extended models.

\section{A. R-parity violation}

If we consider $R$-parity violating terms, the transition operators can be induced at the tree level [65]. The superpotential is

$$
W=\frac{1}{2} \lambda_{i j k} \ell_{i} \cdot \ell_{j} e_{k}^{c},
$$

where $\lambda_{j i k}=-\lambda_{i j k}$. We introduce only $\lambda_{312}$ and $\lambda_{321}$ :

$W=\lambda_{312}\left(\nu_{3} e_{1}-e_{3} \nu_{1}\right) e_{2}^{c}+\lambda_{321}\left(\nu_{3} e_{2}-e_{3} \nu_{2}\right) e_{1}^{c}$,

and Lagrangian contains

$-\mathcal{L} \supset\left(\lambda_{312} \tilde{\nu}_{\tau} \bar{\mu} P_{L} e+\lambda_{321} \tilde{\nu}_{\tau} \bar{e} P_{L} \mu+\right.$ H.c. $)+m_{\tilde{\nu}_{\tau}}^{2}\left|\tilde{\nu}_{\tau}\right|^{2}$, where $m_{\tilde{\nu}_{\tau}}$ is a SUSY breaking tau-sneutrino mass. By the tau-sneutrino exchange, one obtains

$$
\frac{G_{3}}{\sqrt{2}}=\frac{\lambda_{321}^{*} \lambda_{312}}{8 m_{\tilde{\nu}_{\tau}}^{2}} .
$$

The tau-neutrino mass can be generated by loop:

$$
m_{\nu_{\tau}} \sim \frac{\lambda_{231} \lambda_{132}}{16 \pi^{2}} \frac{A m_{e} m_{\mu}}{m_{\tilde{\ell}}^{2}}
$$

where $A$ is a SUSY breaking trilinear scalar coupling.

\section{B. Gaugino contribution}

In this subsection, we describe how large Mu-to- $\overline{\mathrm{Mu}}$ transition can be induced by box contribution in the MSSM with $R$-parity.

Though gauginos are new Majorana particles in the MSSM, the Mu-to- $\overline{\mathrm{Mu}}$ transition is strongly restricted by $\mu \rightarrow e \gamma$ constraints as we have seen several times since the gaugino interactions are $\left(\Delta L_{e}, \Delta L_{\mu}\right)=( \pm 1,0),(0, \pm 1)$ processes. Therefore, it is not very worth describing it in detail, and we here note the rough estimation of the bound of the Mu-to- $\overline{\mathrm{Mu}}$ transition from the $\mu \rightarrow e \gamma$ bound.

Using mass insertion approximation [66,67], the $\mu \rightarrow e \gamma$ constraints of the off-diagonal elements of slepton mass matrix can be written as

$$
\frac{g_{2}^{2} \tan \beta\left|\delta_{12}^{L L}\right|}{m_{\tilde{e}}^{2} G_{F}^{2}} \lesssim O\left(10^{-5}\right)
$$

with

$$
\delta_{12}^{L L}=\left(M_{\tilde{\ell}}^{2}\right)_{12} / m_{\tilde{\ell}}^{2} .
$$

Here, $M_{\tilde{e}}^{2}$ is a $3 \times 3$ SUSY breaking left-handed slepton squared mass matrix, and $m_{\tilde{\ell}}$ is an averaged left-handed slepton mass. This $L L$ constraint mainly comes from the chargino loop diagram, and the neutralino loop can also constrain $\delta_{12}^{R R, L R, R L}$ similarly. The coefficient $G_{1}$ of the transition operator from wino loop can be roughly written as

$$
\frac{G_{1}}{\sqrt{2}} \sim \frac{g_{2}^{4}\left(\delta_{12}^{L L}\right)^{2}}{512 \pi^{2} m_{\tilde{\ell}}^{2}} I_{2}(x, x)+\frac{g_{2}^{4}\left(\delta_{12}^{L L}\right)^{2}}{256 \pi^{2} m_{\tilde{\ell}}^{2}} x I_{1}(x, x),
$$

where $x \simeq m_{\tilde{W}}^{2} / m_{\tilde{\ell}}^{2}$. One should notice that the Mu-to- $\overline{\mathrm{Mu}}$ transition needs the mass insertion twice irrespective of whether the Majorana property of wino is used or not. Because $G_{2}^{b}(x, x) \simeq x G_{1}^{b}(x, x) \lesssim 1$, it is estimated as

$$
\frac{\left|G_{1}\right|}{G_{F}} \lesssim O\left(10^{-8}\right) \frac{g_{2}^{2}\left|\delta_{12}^{L L}\right|}{\tan \beta} .
$$


Therefore, the Mu-to- $\overline{\mathrm{Mu}}$ transition becomes the largest if $\delta_{12}^{L L} \sim 1$ is allowed for the slepton mass to be several times $10 \mathrm{TeV}$. The bino and charged wino box loop contribution with $R R, L R$ mass insertions can be also estimated similarly. The coefficients can depend on the detail of the superparticle spectrum, but we do not survey the numerical detail since the Mu-to- $\overline{\mathrm{Mu}}$ transition is tiny. The chargino and neutralino contribution of $\mu_{R} \rightarrow e_{L} \gamma$ amplitude can be canceled, but the $\mu-e$ conversion is not simultaneously canceled, and thus, it constrains the $\mathrm{Mu}$-to- $\overline{\mathrm{Mu}}$ transition.

\section{Charged Higgsino contribution}

If $\Delta L= \pm 2$ neutrino mass can be utilized, the Mu-to- $\overline{\mathrm{Mu}}$ transition can be potentially generated avoiding the $\mu \rightarrow e \gamma$ constraint. However, as we have seen, the size of neutrino mass restricts the Mu-to- $\overline{\mathrm{Mu}}$ transition. This is because Dirac neutrino masses induce light-heavy neutrino mixings, and large active neutrino masses can be generated at the loop level, even if we adjust the tree-level neutrino masses to be zero. In the situation that the loop-induced mass is suppressed by a flavor symmetry, the box contribution of the Mu-to-Mu transition is also suppressed. In the SUSY limit, the loop-induced masses are zero due to the nonrenormalization theorem. By soft terms of SUSY breaking, the neutrino masses are induced but the size of the loop-induced mass can be suppressed compared to the non-SUSY case [68]. As a result, the Dirac mass or the light-heavy neutrino mixing can be larger than the one in the non-SUSY case, and the Mu-to-Mu transition can be larger. Although it can be larger, it is not large enough to observe in near-future experiments. Therefore, similarly to the non-SUSY model, let us consider a model with inert Higgs doublets and Dirac mass is forbidden by discrete symmetry.
The model we now consider is the SUSY version of the model in Sec. VII B. We introduce additional Higgs doublets $\eta$ and $\eta^{\prime}$ (because of gauge anomaly, we need to introduce a pair of Higgs doubles in the SUSY model), and the Yukawa coupling to $\eta$ with hypercharge $Y=1 / 2$ :

$$
W=y_{\alpha i} \eta \cdot \ell_{\alpha} N_{i}=y_{\alpha i}\left(\eta^{+} e_{\alpha}-\eta^{0} \nu_{\alpha}\right) N_{i} .
$$

We suppose that $\eta^{0}$ does not acquire a vev and the Dirac neutrino mass is absent. In order to avoid too large neutrino masses, we introduce a discrete symmetry to forbid a $\eta \cdot H_{d}$ mass term. In the SUSY model, the so-called $\lambda_{5}$ term $\left(\eta H_{d}\right)^{2}$ is absent in the $F$-term and $D$-term scalar potentials in the SUSY limit, and the neutrino mass shown in Sec. VII $\mathrm{B}$ is not generated. Introducing the $\left(\eta H_{d}\right)^{2}$ term in the superpotential, one obtains the $\lambda_{5}$ term by bino and wino dressing, and the tiny neutrino masses can be generated. Alternatively, one can apply the type-II seesaw to generate the neutrino mass.

The box loop via the charged scalar $\eta^{+}$is the same as in Sec. VII B. We here consider the box loop via the charged Higgsino $\tilde{\eta}^{+}$and sneutrino $\tilde{N}$. The sneutrino masses are given as

$$
-\mathcal{L} \supset\left(m_{\tilde{N}}^{2}\right)_{i j} \tilde{N}_{i} \tilde{N}_{j}^{\dagger}+\left(\frac{1}{2} B_{i j} \tilde{N}_{i} \tilde{N}_{j}+\text { H.c. }\right),
$$

where $m_{\tilde{N}}^{2}$ contains the SUSY breaking squared mass and Majorana mass of $N, 1 / 2 M_{i} N_{i} N_{i}$, and $B_{i j}$ 's are the coefficients of the SUSY breaking bilinear terms. For simplicity, we assume that $\left(m_{\tilde{N}}^{2}\right)_{i j}$ and $B_{i j}$ are flavor diagonal. By a field redefinition, $B_{i i}$ can be made to be real and positive. Then, masses of the sneutrinos for $\sqrt{2} \operatorname{Re} \tilde{N}$ and $\sqrt{2} \operatorname{Im} \tilde{N}$ are $\left(m_{\tilde{N}}^{2}\right)_{i i} \pm B_{i i}$. The coefficient of the Mu-to- $\overline{\mathrm{Mu}}$ transition operator from the sneutrino box loop diagram is obtained as

$$
\begin{aligned}
\frac{G_{1}}{\sqrt{2}}= & \frac{1}{2048 \pi^{2} m_{\tilde{\eta}}^{2}}\left(y_{e i} y_{\mu i}^{*} y_{e j} y_{\mu j}^{*}\left(I_{2}\left(x_{R i}, x_{R j}\right)+I_{2}\left(x_{R i}, x_{I j}\right)+I_{2}\left(x_{I i}, x_{R j}\right)+I_{2}\left(x_{I i}, x_{I j}\right)\right)\right. \\
& \left.+\left(y_{e i}\right)^{2}\left(y_{\mu j}^{*}\right)^{2}\left(I_{2}\left(x_{R i}, x_{R j}\right)-I_{2}\left(x_{R i}, x_{I j}\right)-I_{2}\left(x_{I i}, x_{R j}\right)+I_{2}\left(x_{I i}, x_{I j}\right)\right)\right)
\end{aligned}
$$

where

$$
x_{R i}=\frac{\left(m_{\tilde{N}}^{2}+B\right)_{i i}}{m_{\tilde{\eta}}^{2}}, \quad x_{I i}=\frac{\left(m_{\tilde{N}}^{2}-B\right)_{i i}}{m_{\tilde{\eta}}^{2}} .
$$

We suppose that the Yukawa coupling is given in Eq. (7.32) to eliminate $\mu \rightarrow e \gamma$. Then, the first term of $G_{1}$ in Eq. (9.12) becomes zero, and the second term remains if $B_{i i} \neq 0$ (which reminds us the $B$-term violates lepton number conservation). The Yukawa coupling can generate $\tau \rightarrow \mu(e) \gamma$ :

$$
\operatorname{Br}\left(\tau \rightarrow l_{\alpha} \gamma\right)=\frac{3 \alpha}{16 \pi}\left|y_{\alpha i}^{*} y_{\tau i}\left(\frac{F_{N}\left(x_{R i}\right)+F_{N}\left(x_{I i}\right)}{2 G_{F} m_{\tilde{\eta}}^{2}}-\frac{F_{N}\left(x_{i}\right)}{G_{F} m_{\eta}^{2}}\right)\right|^{2} \operatorname{Br}\left(\tau \rightarrow l_{\alpha} \nu \bar{\nu}\right),
$$


where $x_{i}=M_{i}^{2} / m_{\eta}^{2}$, and the loop function $F_{N}(x)$ is given in Eq. (7.36). The Mu-to- $\overline{\mathrm{Mu}}$ transition can be the largest when $x_{I i} \ll 1 \ll x_{R i}$, and our estimation is $\left|G_{1}\right| / G_{F} \lesssim O\left(10^{-5}\right)$.

\section{DILEPTON GAUGE BOSONS}

In a gauge extension model where the left- and right-handed charged leptons are in one multiplet, a doubly-charged dilepton gauge boson can be introduced [23,69-73]:

$$
\begin{aligned}
\mathcal{L}= & \frac{g_{3 l}}{\sqrt{2}}\left(Y_{\mu}^{++} \overline{\mathrm{e}^{\mathrm{c}}}{ }_{i} \bar{\sigma}^{\mu} \mathrm{e}_{i}+Y_{\mu}^{--} \mathrm{e}^{\mathrm{c}}{ }_{i} \sigma^{\mu} \overline{\mathrm{e}}_{i}\right) \\
& +M_{Y}^{2} Y_{\mu}^{++} Y^{\mu--} .
\end{aligned}
$$

The gauge coupling $g_{3 l}$ is the same as the $S U(2)_{L}$ gauge coupling $g_{2}$ (up to a renormalization correction) in the $S U(3)_{c} \times S U(3)_{L} \times U(1)_{X}$ model. The tree-level exchange of the doubly-charged dilepton gauge boson in Fig. 16 can induce the Mu-to- $\overline{\mathrm{Mu}}$ transition. Integrating out the gauge boson, one obtains

$$
\begin{aligned}
\mathcal{L} & =-\frac{g_{3 l}^{2}}{2 M_{Y}^{2}}\left(\overline{\mathrm{e}^{\mathrm{c}}}{ }_{i} \bar{\sigma}^{\mu} \mathrm{e}_{i}\right)\left(\mathrm{e}^{\mathrm{c}} \sigma_{j} \sigma^{\mu} \overline{\mathrm{e}}_{j}\right) \\
& =-\frac{g_{3 l}^{2}}{M_{Y}^{2}}\left(\overline{\mathrm{e}}_{i}^{\mathrm{c}} \overline{\mathrm{e}}_{j}\right)\left(\mathrm{e}^{\mathrm{c}}{ }_{j} \mathrm{e}_{i}\right)
\end{aligned}
$$

In four-component convention, it is written as

$$
\begin{aligned}
\mathcal{L} & =-\frac{g_{3 l}^{2}}{M_{Y}^{2}}\left(\bar{e}_{j} P_{R} e_{i}\right)\left(\bar{e}_{j} P_{L} e_{i}\right) \\
& =+\frac{g_{3 l}^{2}}{2 M_{Y}^{2}}\left(\bar{e}_{j} \gamma^{\mu} P_{R} e_{i}\right)\left(\bar{e}_{j} \gamma_{\mu} P_{L} e_{i}\right) .
\end{aligned}
$$

Assuming that the current eigenstates $\mathrm{e}_{i}, \mathrm{e}^{\mathrm{c}}{ }_{i}$ correspond to the mass eigenstates of charged leptons, one finds that the transition operator $Q_{3}$ is generated and the coefficient is

$$
\frac{G_{3}}{\sqrt{2}}=-\frac{g_{3 l}^{2}}{8 M_{Y}^{2}} .
$$

The transition bound, Eq. (2.33), implies

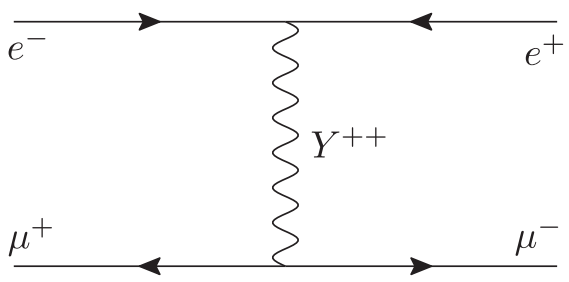

FIG. 16. The tree-level exchange of the doubly charged gauge boson to induce the $\mathrm{Mu}-\mathrm{to}-\overline{\mathrm{Mu}}$ transition.

$$
M_{Y}>1.8 \times \frac{g_{3 l}}{g_{2}} \mathrm{TeV}
$$

In general, the current eigenstates are not the same as the mass eigenstates. Writing the four-component charged leptons of the mass eigenstates as

$$
e_{i}=\left(\begin{array}{c}
\mathrm{e}_{i} \\
\left(V^{\dagger} \overline{\mathrm{e}^{\mathrm{c}}}\right)_{i}
\end{array}\right)
$$

we obtain the gauge interaction as

$$
\begin{array}{ll}
\mathcal{L} \supset & -\frac{g_{3 l}}{2 \sqrt{2}}\left(\frac{V_{i j}-V_{j i}}{2} e_{i}^{T} C \gamma^{\mu} e_{j}+\frac{V_{i j}+V_{j i}}{2} e_{i}^{T} C \gamma^{\mu} \gamma_{5} e_{j}\right) Y_{\mu}^{++} \\
& + \text {H.c. }
\end{array}
$$

Due to the anti-commutation of the fermions, the vector term is anti-symmetric and the axial-vector term is symmetric under the flavor indices. Therefore, LFV decays such as $\mu \rightarrow 3 e$ are induced by the gauge boson exchange in general. If the charged lepton mass matrix $M_{i j}$ is symmetric (where $M_{i j} \mathrm{e}_{i} \mathrm{e}^{\mathrm{C}}{ }_{j}$ is the mass term) and no extra fields are mixed with the charged leptons, one finds that the current eigenstates correspond to the mass eigenstates and $V_{i j}=\delta_{i j}$. Then, LFV decays are not induced by this term.

\section{FLAVORED GAUGE BOSONS}

If there is a neutral gauge boson whose interaction is $\Delta L_{e}=-\Delta L_{\mu}= \pm 1$, the Mu-to- $\overline{\mathrm{Mu}}$ transition can be induced by its exchange at the tree level as in Fig. 17. The gauge boson should have a flavor-dependent charge. For example, the Mu-to- $\overline{\mathrm{Mu}}$ transition in non-Abelian flavor gauge symmetry is discussed in Ref. [74]. We here consider an Abelian flavor gauge symmetry to induce the Mu-to- $\overline{\mathrm{Mu}}$ transition.

As an example, the extra $U(1)$ charges of the lepton doublets $\ell=\left(\nu_{L}, e_{L}\right)$ and scalars $\phi_{i}$ are assigned as in Table I, and the charges of the other fields are zero. We introduce vectorlike lepton doublets $L, \bar{L}$. The mass terms for the first and second generations can be written as

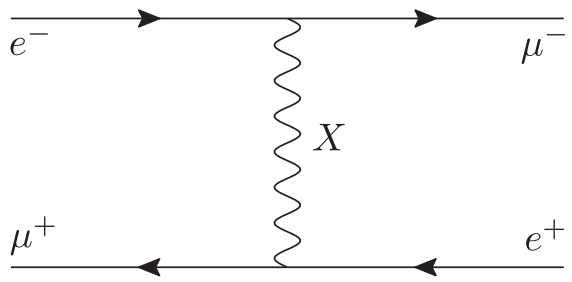

FIG. 17. The tree-level exchange of a neutral gauge boson to induce the $\mathrm{Mu}$-to- $\overline{\mathrm{Mu}}$ transition. 
TABLE I. An example of the extra $U(1)$ charge assignments for the flavored gauge model.

\begin{tabular}{cccc}
\hline \hline$\ell_{1}$ & $\ell_{2}$ & $\phi_{1}$ & $\phi_{2}$ \\
\hline 1 & -1 & -1 & 1 \\
\hline \hline
\end{tabular}

$$
\begin{aligned}
-\mathcal{L}= & y_{1} \phi_{1} \overline{L_{R}} \ell_{1}+y_{2} \phi_{2} \overline{L_{R}} \ell_{2}+M_{L} \bar{L} L+\bar{y}_{1} \overline{e_{1 R}} L_{L} H_{d} \\
& +\bar{y}_{2} \overline{e_{2 R}} L_{L} H_{d} .
\end{aligned}
$$

Integrating out the vectorlike lepton doublets, we obtain the Yukawa interactions of the first and second generations of charged leptons. The electron and muon mass eigenstates are given by

$$
\begin{aligned}
e_{L} & =\cos \theta e_{1}+\sin \theta e_{2}, \\
\mu_{L} & =-\sin \theta e_{1}+\cos \theta e_{2},
\end{aligned}
$$

where $\tan \theta=-y_{1} \phi_{1} /\left(y_{2} \phi_{2}\right)$. Denoting the extra $U(1)$ gauge field as $X_{\mu}$, we can write the gauge interaction as

$$
\mathcal{L}=g_{X} X_{\mu}\left(\bar{\ell}_{1} \gamma^{\mu} \ell_{1}-\bar{\ell}_{2} \gamma^{\mu} \ell_{2}\right)+\frac{1}{2} M_{X}^{2} X_{\mu} X^{\mu}
$$

The gauge interaction of the electron and muon is

$$
\begin{aligned}
g_{X} X_{\mu} & \left(\bar{e}_{1} \gamma^{\mu} e_{1}-\bar{e}_{2} \gamma^{\mu} e_{2}\right) \\
= & g_{X} X_{\mu}\left(\left(\bar{e}_{L} \gamma^{\mu} \mu_{L}+\bar{\mu}_{L} \gamma^{\mu} e_{L}\right) \sin 2 \theta\right. \\
& \left.+\left(\bar{e}_{L} \gamma^{\mu} e_{L}-\bar{\mu}_{L} \gamma^{\mu} \mu_{L}\right) \cos 2 \theta\right) .
\end{aligned}
$$

The $\sin 2 \theta$ term can induce the Mu-to- $\overline{\mathrm{Mu}}$ transition,

$$
\mathcal{L} \supset-\frac{g_{X}^{2} \sin ^{2} 2 \theta}{2 M_{X}^{2}}\left(\bar{\mu}_{L} \gamma_{\mu} e_{L}\right)\left(\bar{\mu}_{L} \gamma^{\mu} e_{L}\right)
$$

and

$$
\frac{G_{1}}{\sqrt{2}}=\frac{g_{X}^{2} \sin ^{2} 2 \theta}{8 M_{X}^{2}} .
$$

We need to assume $|\cos 2 \theta| \ll 1$ to suppress $\mu \rightarrow 3 e$. (Surely, we assume that the mixings of $X$ to photon and $Z$ boson are negligibly small). Naively, $\operatorname{Br}(\mu \rightarrow 3 e)<10^{-12}$ provides a bound:

$$
\frac{G_{1}}{G_{F}}|\cot 2 \theta| \lesssim \frac{1}{2 \sqrt{2}} \times 10^{-6}
$$

Since the signs of $e e$ and $\mu \mu$ couplings to $X_{\mu}$ in the above are opposite, the constraint of $\cos 2 \theta$ from $\mu \rightarrow e \gamma$ is loose for $m_{e}, m_{\mu} \ll M_{X}$. Theoretically, if there is an exchange symmetry $\phi_{1} \leftrightarrow \phi_{2}, \ell_{1} \leftrightarrow \ell_{2}$ in the Lagrangian, one finds that $|\tan \theta|=1$ and the unwanted LFV is absent.

One can similarly consider a model where $X_{\mu}$ can couple to both left- and right-handed electron and muons. In that model, the bounds of the diagonal couplings to electrons and muons are stronger. The right-handed operators for $\mu \rightarrow 3 e$ are simply added at the tree level, while the bound becomes stronger due to the chirality flipping in the internal line for $\mu \rightarrow e \gamma$. In any cases, the models are free from LFV constraints by a choice that the diagonal couplings are absent. Even in the choice, the electron and muon $g-2$ can be induced. If $X_{\mu}$ couples only to the left-handed leptons (as the example above), the ratio of the induced anomalous magnetic moments ${ }^{6}$ is

$$
\frac{\Delta a_{e}}{\Delta a_{\mu}}=\frac{m_{e}^{2}}{m_{\mu}^{2}}
$$

If $X_{\mu}$ couples to both left- and right-handed ones, the muon and electron mass can be hit at internal lines, and thus, the above flavor relation is violated. One can find that the magnitude of $\Delta a_{e} / m_{e}^{2}$ can be much larger than $\Delta a_{\mu} / m_{\mu}^{2}$.

Let us consider the model where both left- and righthanded leptons couple to the extra gauge boson in the absence of the diagonal couplings:

$$
\mathcal{L}=g_{X} X_{\mu}\left(\overline{e_{L}} \gamma^{\mu} \mu_{L}+a \overline{e_{R}} \gamma^{\mu} \mu_{R}+(\mu \leftrightarrow e)\right),
$$

where $a$ is a $U(1)$ charge for the right-handed charged leptons. In this case, we obtain

$\frac{G_{1}}{\sqrt{2}}=\frac{g_{X}^{2}}{8 M_{X}^{2}}, \quad \frac{G_{2}}{\sqrt{2}}=\frac{a^{2} g_{X}^{2}}{8 M_{X}^{2}}, \quad \frac{G_{3}}{\sqrt{2}}=\frac{2 a g_{X}^{2}}{8 M_{X}^{2}}$.

The contributions to $(g-2) / 2$ of the muon and electron can be calculated as

$$
\begin{gathered}
\Delta a_{\mu}=-m_{\mu}^{2} \frac{\left(1+a^{2}\right) g_{X}^{2}}{32 \pi^{2} M_{X}^{2}} \times \frac{8}{3}, \\
\Delta a_{e}=m_{e} m_{\mu} \frac{2 a g_{X}^{2}}{32 \pi^{2} M_{X}^{2}} G_{X}\left(\frac{m_{\mu}^{2}}{M_{X}^{2}}\right),
\end{gathered}
$$

${ }^{6}$ The current muon $g-2$ measurement [75] implies

$$
\Delta a_{\mu}=(2.51 \pm 0.59) \times 10^{-9} .
$$

The values of the anomalous electron $g-2$ are reported by Berkeley [76] and Laboratoire Kastler Brossel (LKB) [77] groups:

$$
\begin{aligned}
\Delta a_{e}(\text { Berkeley }) & =(-8.8 \pm 3.6) \times 10^{-13}, \\
\Delta a_{e}(\mathrm{LKB}) & =(4.8 \pm 3.0) \times 10^{-13},
\end{aligned}
$$

which seem to depend on the fine structure constant extracted from the Rydberg constant. 


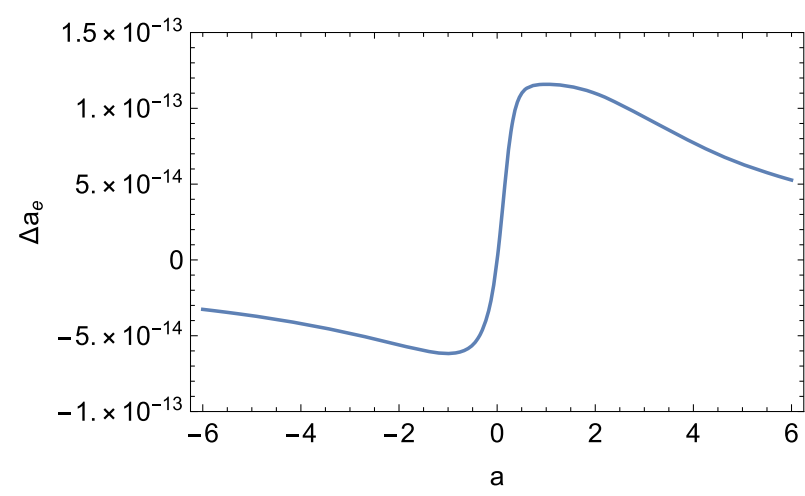

FIG. 18. The contribution to the electron $g-2\left(\Delta a_{e}\right)$ is shown as a function of the $U(1)$ charge $a$ for the right-handed charged leptons when the Mu-to- $\overline{\mathrm{Mu}}$ transition is assumed to be just the same as the experimental upper bound.

where the loop function is

$$
G_{X}(x)=\frac{4-3 x-x^{3}+6 x \ln x}{(1-x)^{3}} .
$$

The contribution of the neutral gauge boson to the muon $g-2$ is negative. The experimental bound of the Mu-to- $\overline{\mathrm{Mu}}$ transition implies

$$
-\Delta a_{\mu} \lesssim 2 \times 10^{-11}
$$

Therefore, the muon $g-2$ anomaly cannot be explained. In Fig. 18, we show the electron $g-2$ as a function of $a$ when the Mu-to- $\overline{\mathrm{Mu}}$ transition is just on the current experimental bound. The magnitude of the electron $g-2$ is one digit smaller than the current central value. Though the electron $g-2$ can be larger than the naive expectation without flavor violation, the $\mathrm{Mu}$-to- $\overline{\mathrm{Mu}}$ transition constrains the magnitude of it.

\section{OTHER MODELS}

\section{A. Leptoquark}

If there are $\bar{F} P_{L} \ell_{i} S$ and/or $\bar{F} P_{R} e_{i} S$ types of interactions ( $F$ : fermion, $S$ : boson), the Mu-to- $\overline{\mathrm{Mu}}$ transition can be induced by a box diagram. As an example, we consider a "leptoquark" scalar,

$$
D:\left(\mathbf{3}, \mathbf{1},-\frac{1}{3}\right)
$$

and we allow the following interactions ${ }^{7}$ :

$$
-\mathcal{L}=\bar{y}_{i j} \ell_{i} q_{j} D^{*}+y_{i j} e_{i}^{c} u_{j}^{c} D+\text { H.c. }
$$

\footnotetext{
${ }^{7}$ In this section, we use two-component fermion convention to avoid complicated expressions.
}

We must forbid "diquark" interactions $\left(q q D\right.$ and $u^{c} d^{c} D^{*}$ terms) to avoid a rapid proton decay. There is a fairly merit to introduce the leptoquark coupling: it induces the $b \rightarrow s e^{+} e^{-}, b \rightarrow s \mu^{+} \mu^{-}$for lepton nonuniversality at the tree level [78,79].

Let us consider $\mu \rightarrow e \gamma$ constraints. We suppose $y_{i j} \rightarrow 0$. Then, the bound is roughly

$$
\frac{\bar{y}_{1 i}^{*} \bar{y}_{2 i}}{M_{D}^{2} G_{F}} F_{1} \lesssim O\left(10^{-5}\right)
$$

where $F_{1}$ is a form factor. If both $y_{i j}$ and $\bar{y}_{i j}$ are switched on, the chirality can flip at an internal line, and the bound can be roughly

$$
\frac{m_{u_{i}}}{m_{\mu}} \frac{y_{1 i} \bar{y}_{2 i}}{M_{D}^{2} G_{F}} F_{2} \lesssim O\left(10^{-5}\right),
$$

where $F_{2}$ is a form factor. Therefore, for $y, \bar{y}<O(1)$, the $\mu \rightarrow e \gamma$ constraints roughly give the bound,

$$
\frac{\left|G_{1,2,3}\right|}{G_{F}} \lesssim O\left(10^{-8}\right) .
$$

This is a consequence when only $\left(\Delta L_{e}, \Delta L_{\mu}\right)=( \pm 1,0)$, $(0, \pm 1)$ interactions are introduced.

\section{B. Vectorlike fermions}

Vectorlike fermions are often introduced in flavor models. Via the box diagram in which the vectorlike fermions propagate, the Mu-to- $\overline{\mathrm{Mu}}$ transition can be generated. Here, we consider a minimal version to illustrate the essence. We introduce $S U(2)_{L}$ singlet fermions:

$$
\bar{E}^{c}:(\mathbf{1}, \mathbf{1},-1), \quad E^{c}:(\mathbf{1}, \mathbf{1}, 1),
$$

and SM singlet flavon complex scalars $\phi_{e}$ and $\phi_{\mu}$. The global $U(1)$ charges are assigned as in Table II, and Lagrangian is

$$
\begin{aligned}
-\mathcal{L}= & h_{e} \phi_{e} e^{c} \bar{E}^{c}+h_{\mu} \phi_{\mu} \mu^{c} \bar{E}^{c} \\
& +\sum_{i=1,2} y_{i} \ell_{i} H E^{c}+M_{E} \bar{E}^{c} E^{c} .
\end{aligned}
$$

The mass matrix is given as

TABLE II. The global $U(1)$ charge assignments for the model with vectorlike fermions discussed in Sec. XII B.

\begin{tabular}{cccccc}
\hline \hline$e^{c}$ & $\mu^{c}$ & $\phi_{e}$ & $\phi_{\mu}$ & $\bar{E}^{c}$ & $E^{c}$ \\
\hline$a$ & $b$ & $-a$ & $-b$ & 0 & 0 \\
\hline \hline
\end{tabular}




$$
-\mathcal{L} \supset\left(\begin{array}{lll}
e & \mu & \bar{E}^{c}
\end{array}\right)\left(\begin{array}{ccc}
0 & 0 & y_{1} v \\
0 & 0 & y_{2} v \\
h_{e} \phi_{e} & h_{\mu} \phi_{\mu} & M_{E}
\end{array}\right)\left(\begin{array}{l}
e^{c} \\
\mu^{c} \\
E^{c}
\end{array}\right) .
$$

The muon mass is generated as

$$
m_{\mu} \simeq \frac{\sqrt{y_{1}^{2}+y_{2}^{2}} \sqrt{\left(h_{e} \phi_{e}\right)^{2}+\left(h_{\mu} \phi_{\mu}\right)^{2}}}{M_{E}} v .
$$

Even if the Yukawa couplings are $O(1)$, the smallness of muon mass is explained by the vev hierarchy $\phi_{e} \ll \phi_{\mu} \ll M_{E}$. Surely, the electron is massless at this stage, unless we introduce additional heavier vectorlike fermions. To suppress $\mu \rightarrow e \gamma$ via the vectorlike fermions, $\phi_{e} \ll \phi_{\mu}$ is needed.

The $\phi_{e}$ field has partial lepton numbers as $\left(L_{e}, L_{\mu}\right)=(-1,1)$. Once $\phi_{e}$ and $\phi_{\mu}$ acquire vevs, partial lepton number symmetry is broken (while total lepton number symmetry is kept). Then, the Mu-to- $\overline{\mathrm{Mu}}$ transition can be generated as

$$
\begin{aligned}
\frac{G_{2}}{\sqrt{2}}= & \frac{h_{e}^{* 2} h_{\mu}^{2}}{2048 \pi^{2} M_{E}^{2}}\left(I_{2}\left(x_{\mathrm{Re}}, x_{R \mu}\right)-I_{2}\left(x_{\mathrm{Re}}, x_{I \mu}\right)\right. \\
& \left.-I_{2}\left(x_{I e}, x_{R \mu}\right)+I_{2}\left(x_{I e}, x_{I \mu}\right)\right),
\end{aligned}
$$

where

$$
x_{R \alpha}=\frac{m_{\operatorname{Re} \phi_{\alpha}}^{2}}{M_{E}^{2}}, \quad x_{I \alpha}=\frac{m_{\operatorname{Im} \phi_{\alpha}}^{2}}{M_{E}^{2}} .
$$

If soft breaking terms of the global $U(1)$ symmetry are absent, the imaginary parts are massless. Because the masses of real and imaginary parts split, $G_{2}$ becomes nonzero.

\section{Axionlike particle}

If a global flavor symmetry is broken spontaneously, the following flavored axionlike coupling can be considered [80-82]:

$$
\mathcal{L}=\frac{\partial_{\mu} a}{2 f_{a}} \bar{f}_{i} \gamma^{\mu}\left(v_{i j}+\gamma_{5} a_{i j}\right) f_{j}
$$

By a field redefinition $f$, one can consider the axionlike coupling as

$$
a \bar{f}\left(y_{i j}^{S}+\gamma_{5} y_{i j}^{P}\right) f .
$$

Therefore, one can think that the coupling can be a global symmetry version of the model in Sec. XI, or the Higgs scalar has a Peccei-Quinn-like global charge in the model in Sec. VIII. The Mu-to- $\overline{\mathrm{Mu}}$ transition can be induced by the exchange of the axionlike particle at the tree level. The Mu-to- $\overline{\mathrm{Mu}}$ transition induced by the axionlike particle is intensively discussed in Ref. [82].

\section{CONCLUSION}

The Mu-to- $\overline{\mathrm{Mu}}$ transition is one of the interesting probes for physics beyond the SM. In near future, the search experiments will be performed in some facilities, such as J-PARC in Japan and CSNS in China. In anticipation of those upcoming experiments, we have evaluated their impact on models and the connection to other experiments. Assuming appropriate mediators, we are allowed to consider five independent effective operators to induce the transition. In terms of the effective couplings, we have shown the general formula of the transition probability in the zero or nonzero magnetic field. We have pointed out that the magnetic-field dependence helps us to identify the type of dominant effective operators.

For each plausible new physics model, we have estimated the maximum size of the induced effective couplings $G_{i}(i=1-5)$ given in Eq. (2.18), taking into account current experimental constraints. The result shows that the Mu-to- $\overline{\mathrm{Mu}}$ transition is most sensitive to the cases where the mediator has $\Delta L_{e}-\Delta L_{\mu}= \pm 2$ interactions in the model: such as a doubly charged scalar, doubly charged gauge boson, neutral scalar, and neutral gauge boson. Since the transition is generated at the tree level by the exchange of the mediator, the size of the effective couplings can be $\left|G_{i}\right| / G_{F} \lesssim O\left(10^{-3}\right)$, which is as large as the current experimental bound (See Eqs. (2.29)-(2.33) for the bound). The severe experimental constraints from LFV decays such as $\mu \rightarrow e \gamma$ and $\mu \rightarrow 3 e$ can be avoided for the mediators with the $\Delta L_{e}-\Delta L_{\mu}= \pm 2$ interactions. The left-right model with $S U(2)_{R}$ triplet is typically the case, for example. To generate the sizable Mu-to- $\overline{\mathrm{Mu}}$ transition avoiding the LFV decays, one needs some ideas such as introducing a discrete flavor symmetry. Thus, the possible observation of the Mu-to- $\overline{\mathrm{Mu}}$ transition at the near-future experiment can give us the drastic paradigm change of the understanding of the lepton flavors.

In the models with $\Delta L_{e}-\Delta L_{\mu}= \pm 1$ interactions, the Mu-to- $\overline{\mathrm{Mu}}$ transition can be generated by box loop diagrams. However, the $\mu \rightarrow e \gamma$ constraint severely bounds the transition, and we have obtained $\left|G_{i}\right| / G_{F} \lesssim O\left(10^{-8}\right)$ at most.

If mass terms violate the (partial) lepton numbers, the Mu-to- $\overline{\mathrm{Mu}}$ transition can be generated by box loop diagrams even without the LFV interactions. The light-heavy neutrino mixing needs to be large in order to enlarge the $\mathrm{Mu}$-to- $\overline{\mathrm{Mu}}$ transition induced by the lepton number violation. However, the large light-heavy neutrino mixing can induce sizable active neutrino masses radiatively, which spoils the realization of the sub-eV neutrino mass. In the sense, the Mu-to- $\overline{\mathrm{Mu}}$ transition induced by the lepton 
number violation is conceptually constrained to realize the sub-eV neutrino masses. We need to assume the neutrino masses to be zero by a discrete symmetry at the tree level, and the sub-eV active neutrino masses are generated radiatively to obtain a large size of the Mu-to- $\overline{\mathrm{Mu}}$ transition. We have found $\left|G_{i}\right| / G_{F} \lesssim O\left(10^{-5}\right)$ in such models.

The existence of the doubly charged scalar can be related to the neutrino mass generation. As examples of such models, we have investigated the type-II seesaw, Zee-Babu, and cocktail models. The Zee-Babu model especially leaves the possibility of $\left|G_{2}\right| / G_{F} \sim O\left(10^{-3}\right)$, which is related to $\tau^{-} \rightarrow \mu^{+} \mu^{-} \mu^{-}$and $\tau^{-} \rightarrow \mu^{+} e^{-} e^{-}$. In the typeII seesaw model, the large Mu-to- $\overline{\mathrm{Mu}}$ transition favors degenerate neutrino masses, which results in $\left|G_{1}\right| / G_{F} \lesssim$ $O\left(10^{-5}\right)$ if the cosmological neutrino mass bound is applied. The cocktail model predicts $\left|G_{2}\right| / G_{F} \lesssim O\left(10^{-5}\right)$ due to the constraints of $\tau^{-} \rightarrow e^{+} e^{-} e^{-}$and $\tau^{-} \rightarrow e^{+} \mu^{-} \mu^{-}$.

The Mu-to- $\overline{\mathrm{Mu}}$ transition is model-independently related to the measurements of the Mu HFS interval by the effective couplings. Although the current sensitivity of the HFS interval is less than that of the Mu-to- $\overline{\mathrm{Mu}}$ transition, future excellent upgrades will have the potential to provide us with a way to check the Mu-to- $\overline{\mathrm{Mu}}$ transition. Furthermore, through specified models, the Mu-to- $\overline{\mathrm{Mu}}$ transition is connected to the other observables in flavor physics (e.g., neutrino masses, muon/electron $g-2$, LFV searches, and so on) and direct particle searches by collider experiments. The near-future experiments for the $\mathrm{Mu}$-to- $\overline{\mathrm{Mu}}$ transition will give us complementary information to investigate the detailed structure of high-energy physics.

We conclude with model-by-model tables of the Mu-to- $\overline{\mathrm{Mu}}$ transition for the convenience of readers, though there are some overlaps with what we have already described in this section. In Table III, we list the models in which the Mu-to- $\overline{\mathrm{Mu}}$ transition operators are generated at the tree-level and they are tested by the near-future $\mathrm{Mu}$-to- $\overline{\mathrm{Mu}}$ transition experiments. In Table IV, we list the models in which the transition operators are generated by box diagrams via $\Delta L_{e}-\Delta L_{\mu}= \pm 1$ interactions, and the size of the transitions is suppressed by the constraints from LFV decays. If the active neutrino masses are generated radiatively, the lepton number violation is utilized to generate the $\mathrm{Mu}$-to- $\overline{\mathrm{Mu}}$ transitions by box diagrams avoiding the constraints from LFV decays. We list those models in Table V. In Table VI, we list the predictive neutrino mass models with doubly charged scalars which can induce the Mu-to- $\overline{\mathrm{Mu}}$ transitions at the tree level.

TABLE III. The models to generate the Mu-to- $\overline{\mathrm{Mu}}$ transition at the tree level and the corresponding coefficients $G_{i}$. For each model, a checkmark $\checkmark$ is placed in the column where the coefficient can be obtained around the current experimental bound $\left(\left|G_{i}\right| / G_{F} \sim O\left(10^{-3}\right)\right)$. In the column where the coefficient that is generated but becomes smaller due to LFV bounds, a triangle mark $\triangle$ is placed. The section column contains the section number in which the model is described.

\begin{tabular}{lcccccc}
\hline \hline Model & $G_{1}$ & $G_{2}$ & $G_{3}$ & $G_{4}$ & $G_{5}$ & Section \\
\hline Type I + II hybrid seesaw & $\checkmark$ & $\ldots$ & $\ldots$ & $\ldots$ & $\ldots$ & V \\
Left-right model with $S U(2)_{R}$ triplet & $\ldots$ & $\checkmark$ & $\ldots$ & $\ldots$ & $\ldots$ & VI B \\
Inert Higgs doublet & $\ldots$ & $\ldots$ & $\checkmark$ & $\triangle$ & $\triangle$ & VIII \\
$R$-parity violating SUSY & $\ldots$ & $\ldots$ & $\checkmark$ & $\ldots$ & $\ldots$ & IX A \\
Dilepton gauge boson & $\ldots$ & $\ldots$ & $\checkmark$ & $\ldots$ & $\ldots$ & X \\
Neutral flavor gauge boson & $\checkmark$ & $\checkmark$ & $\checkmark$ & $\ldots$ & $\ldots$ & XI \\
\hline \hline
\end{tabular}

TABLE IV. The models in which the Mu-to- $\overline{\mathrm{Mu}}$ transitions are induced at the one-loop level by $\Delta L_{e}-\Delta L_{\mu}= \pm 1$ interactions, and the estimation of the corresponding coefficients. Due to the LFV constraints, the induced size is tiny in this category of the interaction.

\begin{tabular}{|c|c|c|c|c|}
\hline Model & $\left|G_{1}\right| / G_{F}$ & $\left|G_{2}\right| / G_{F}$ & $\left|G_{3}\right| / G_{F}$ & Section \\
\hline Heavy singlet neutrino & $\lesssim O\left(10^{-8}\right)$ & 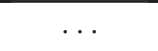 & & IV \\
\hline Left-right model without $S U(2)_{R}$ triplet & $\lesssim O\left(10^{-8}\right)$ & $\lesssim O\left(10^{-8}\right)$ & $\lesssim O\left(10^{-10}\right)$ & VI A \\
\hline SUSY (Gaugino loop) & $\lesssim O\left(10^{-8}\right)$ & $\ldots$ & $\ldots$ & IX B \\
\hline Leptoquark & $\lesssim O\left(10^{-8}\right)$ & $\lesssim O\left(10^{-8}\right)$ & $\lesssim O\left(10^{-8}\right)$ & XII A \\
\hline
\end{tabular}


TABLE V. The radiative neutrino mass models in which the Mu-to- $\overline{\mathrm{Mu}}$ transitions are induced at the one-loop level by using the lepton number violation. The constraints from LFV decays via $\Delta L_{e}-\Delta L_{\mu}= \pm 1$ interactions can be avoided. ${ }^{(*)}$ In order to realize the size of the active neutrino masses in the KNT model, one needs to consider a nonperturbative region of the couplings. Then, the induced size of the coefficient can be larger, but the quality of the estimate is different from others.

\begin{tabular}{lccc}
\hline \hline Model & $\left|G_{1}\right| / G_{F}$ & $\left|G_{2}\right| / G_{F}$ & Section \\
\hline Charged Higgs(ino) & $\lesssim O\left(10^{-5}\right)$ & $\ldots$ & VII B, IX C \\
KNT model & $\cdots$ & $\lesssim O\left(10^{-5}\right)^{(*)}$ & VII C 1 \\
AKS model & $\cdots$ & $\lesssim O\left(10^{-6}\right)$ & VII C 2 \\
\hline \hline
\end{tabular}

TABLE VI. The neutrino mass models in which the Mu-to- $\overline{\mathrm{Mu}}$ transitions are induced at the tree level via $\Delta L_{e}-\Delta L_{\mu}= \pm 2$ interactions with doubly charged scalars. ${ }^{(\#)}$ In the type-II seesaw, the induced size of the coefficient is bounded by the absolute neutrino mass from the cosmological measurements. If neutrinos are not stable in the cosmological time, the bound is not applied and the coefficient of $\left|G_{1}\right|$ can be larger.

\begin{tabular}{lccr}
\hline \hline Model & $\left|G_{1}\right| / G_{F}$ & $\left|G_{2}\right| / G_{F}$ & Section \\
\hline Type-II seesaw & $\lesssim O\left(10^{-5}\right)^{(\#)}$ & $\ldots$ & V \\
Zee-Babu model & $\ldots$ & $\lesssim O\left(10^{-3}\right)$ & VII A 1 \\
Cocktail model & $\ldots$ & $\lesssim O\left(10^{-5}\right)$ & VII A 2 \\
\hline \hline
\end{tabular}

\section{ACKNOWLEDGMENTS}

We would like to thank N. Kawamura and K. Shimomura for their useful comments. This work was supported by JSPS KAKENHI Grants No. JP18H01210 and No. JP21H00081 (Y. U.).

\section{APPENDIX A: TRANSITION AMPLITUDE}

Here, we show a calculation for the amplitude of the Mu-to-Mu transition [14].

The charged lepton fields $l=\mu, e$ included in the operators, $Q_{i}$ in Eqs. (2.9)-(2.13), are explicitly written as

$$
l(x)=\int \frac{d^{3} p}{(2 \pi)^{3}} \frac{1}{\sqrt{2 E_{p}}} \sum_{s}\left\{a_{l}^{p, s} u_{l}^{s}(p) \exp (-i p x)+b_{l}^{p, s \dagger} v_{l}^{s}(p) \exp (i p x)\right\},
$$

where $u_{l}^{s}(p)$ and $v_{l}^{s}(p)$ are the four-component Dirac spinors. In the nonrelativistic limit, the spinors are given as

$$
u_{l}^{s}=\sqrt{m_{l}}\left(\begin{array}{c}
\xi^{s} \\
\xi^{s}
\end{array}\right), \quad v_{l}^{s}=\sqrt{m_{l}}\left(\begin{array}{c}
\eta^{s} \\
-\eta^{s}
\end{array}\right)
$$

where $\xi^{+1 / 2}=(1,0)^{T}, \xi^{-1 / 2}=(0,1)^{T}, \eta^{+1 / 2}=(0,1)^{T}$, and $\eta^{-1 / 2}=(-1,0)^{T}$. The creation and annihilation operators satisfy the anticommutation relation,

$$
\left\{a_{l}^{\boldsymbol{p}, s}, a_{l^{\prime}}^{\boldsymbol{p}^{\prime}, s^{\prime} \dagger}\right\}=\left\{b_{l}^{\boldsymbol{p}, s}, b_{l^{\prime}}^{\boldsymbol{p}^{\prime},,^{\prime} \dagger}\right\}=(2 \pi)^{3} \delta^{(3)}\left(\boldsymbol{p}-\boldsymbol{p}^{\prime}\right) \delta_{s, s^{\prime}} \delta_{l, l^{\prime}}
$$

Using the creation operators, we define the Mu state as

$$
|\operatorname{Mu}(\boldsymbol{P}) ; F, m\rangle=\sum_{s_{e}, s_{\mu}}\left(1 / 2, s_{\mu}, 1 / 2, s_{e} \mid F, m\right) \int \frac{d^{3} q}{(2 \pi)^{3}} \tilde{\varphi}(\boldsymbol{q}) a_{e}^{\boldsymbol{p}_{e}, s_{e}^{\dagger}} b_{\mu}^{\boldsymbol{p}_{\mu}, s_{\mu}^{\dagger}}|0\rangle,
$$

where $\boldsymbol{P}$ and $\boldsymbol{q}$ are the total and relative momentums of the muon-electron system, respectively, so that $\boldsymbol{p}_{e}=$ $m_{e} /\left(m_{e}+m_{\mu}\right) \boldsymbol{P}+\boldsymbol{q}$ and $\boldsymbol{p}_{\mu}=m_{\mu} /\left(m_{e}+m_{\mu}\right) \boldsymbol{P}-\boldsymbol{q}$. Here, $\left(1 / 2, s_{\mu}, 1 / 2, s_{e} \mid F, m\right)$ is the Clebsch-Gordan coefficient. The wave function $\tilde{\varphi}(\boldsymbol{q})$ of the ground state in the momentum space is normalized to be 


$$
\int \frac{d^{3} q}{(2 \pi)^{3}}|\tilde{\varphi}(\boldsymbol{q})|^{2}=1
$$

With the above definition, the $\mathrm{Mu}$ state obeys

$$
\left\langle\operatorname{Mu}(\boldsymbol{P}) ; F, m \mid \operatorname{Mu}\left(\boldsymbol{P}^{\prime}\right) ; F^{\prime}, m^{\prime}\right\rangle=(2 \pi)^{3} \delta^{(3)}\left(\boldsymbol{P}-\boldsymbol{P}^{\prime}\right) \delta_{F, F^{\prime}} \delta_{m, m^{\prime}}
$$

On the other hand, the $\overline{\mathrm{Mu}}$ state is defined by the charge conjugation of Eq. (A4), ${ }^{8}$

$$
|\overline{\mathrm{Mu}}(\boldsymbol{P}) ; F, m\rangle=\sum_{s_{e}, s_{\mu}}\left(1 / 2, s_{\mu}, 1 / 2, s_{e} \mid F, m\right) \int \frac{d^{3} q}{(2 \pi)^{3}} \tilde{\varphi}(\boldsymbol{q}) b_{e}^{\boldsymbol{p}_{e}, s_{e}^{\dagger}} a_{\mu}^{\boldsymbol{p}_{\mu}, s_{\mu}^{\dagger}}|0\rangle .
$$

We show the calculation for the transition amplitude via the operator $Q_{1}$,

$$
\left\langle\overline{\mathrm{Mu}} ; F, m\left|Q_{1}\right| \mathrm{Mu} ; F, m\right\rangle=\left\langle\overline{\mathrm{Mu}} ; F, m\left|\left(\bar{\mu} \gamma_{\mu}\left(1-\gamma_{5}\right) e\right)\left(\bar{\mu} \gamma^{\mu}\left(1-\gamma_{5}\right) e\right)\right| \mathrm{Mu} ; F, m\right\rangle,
$$

as an example. By substituting the explicit form of the field into the equation and using the anticommutation relation, we obtain

$$
\begin{aligned}
\left\langle\overline{\mathrm{Mu}} ; F, m\left|Q_{1}\right| \mathrm{Mu} ; F, m\right\rangle= & \frac{|\varphi(0)|^{2}}{m_{\mu} m_{e}} \sum_{\bar{s}_{e} \bar{s}_{\mu}}\left(1 / 2, \bar{s}_{\mu}, 1 / 2, \bar{s}_{e} \mid F, m\right) \sum_{s_{e}, s_{\mu}}\left(1 / 2, s_{\mu}, 1 / 2, s_{e} \mid F, m\right) \\
& \times\left[-\left(\bar{u}_{\mu}^{\bar{s}_{\mu}} \gamma_{\alpha} P_{L} v_{e}^{\bar{s}_{e}}\right)\left(\bar{v}_{\mu}^{s_{\mu}} \gamma^{\alpha} P_{L} u_{e}^{s_{e}}\right)-\left(\bar{v}_{\mu}^{s_{\mu}} \gamma_{\alpha} P_{L} u_{e}^{\bar{s}_{e}}\right)\left(\bar{u}_{\mu} \gamma^{\alpha} P_{L} v_{e}^{s_{e}}\right)\right. \\
& \left.+\left(\bar{v}_{\mu}^{s_{\mu}} \gamma_{\alpha} P_{L} \bar{s}_{e}\right)\left(\bar{u}_{\mu}^{\bar{s}_{\mu}} \gamma^{\alpha} P_{L} u_{e}^{s_{e}}\right)+\left(\bar{u}_{\mu}^{\bar{s}_{\mu}} \gamma_{\alpha} P_{L} u_{e}^{s_{e}}\right)\left(\bar{v}_{\mu}^{s_{\mu}} \gamma^{\alpha} P_{L} \bar{s}_{e}\right)\right],
\end{aligned}
$$

where $\varphi$ is the wave function in the coordinate space,

$$
\varphi(\boldsymbol{r})=\int \frac{d^{3} q}{(2 \pi)^{3}} \tilde{\varphi}(\boldsymbol{q}) \exp (i \boldsymbol{q} \cdot \boldsymbol{r})
$$

The first term in the square bracket of Eq. (A9) reduces to

$$
\left(\bar{u}_{\mu}^{\bar{s}_{\mu}} \gamma_{\alpha} P_{L} v_{e}^{\bar{s}_{e}}\right)\left(\bar{v}_{\mu}^{s_{\mu}} \gamma^{\alpha} P_{L} u_{e}^{s_{e}}\right)=m_{\mu} m_{e} \operatorname{Tr}\left[\eta^{\bar{s}_{e}} \bar{\xi}^{\bar{s}_{\mu} \dagger} \bar{\sigma}_{\alpha}\right] \operatorname{Tr}\left[\xi^{s_{e}} \eta^{s_{\mu}^{\dagger}} \bar{\sigma}^{\alpha}\right]
$$

where $\bar{\sigma}^{\alpha}=\left(\sigma_{0}^{0},-\sigma_{1}^{i}\right)$ and $\sigma_{0}^{0}$ is the $2 \times 2$ identity matrix, and $\sigma_{1}^{i}(i=1,2,3)$ is the Pauli matrix. The products of spinors, $\eta$ and $\xi$, can be replaced with the Pauli matrices by

$$
\begin{gathered}
\sum_{s_{e}, s_{\mu}}\left(1 / 2, s_{\mu}, 1 / 2, s_{e} \mid F, m\right) \xi^{s_{e}} \eta^{s_{\mu}^{\dagger}}=\frac{\bar{\sigma}_{F}^{m}}{\sqrt{2}}, \\
\sum_{\bar{s}_{e}, \bar{s}_{\mu}}\left(1 / 2, \bar{s}_{\mu}, 1 / 2, \bar{s}_{e} \mid F, m\right) \eta^{\bar{s}_{e}} \xi^{\bar{s}_{\mu}^{\dagger}}=-\frac{\sigma_{F}^{m}}{\sqrt{2}},
\end{gathered}
$$

where $\sigma_{0}^{0}=\bar{\sigma}_{0}^{0}$ is the $2 \times 2$ identity matrix, and $\sigma_{1}^{m}(m=0, \pm 1)$ in those equations represents the Pauli matrix in the spherical basis: $\sigma_{1}^{ \pm 1}=-\bar{\sigma}_{1}^{ \pm 1}=\left(\mp \sigma_{1}-i \sigma_{2}\right) / \sqrt{2}$, and $\sigma_{1}^{0}=-\bar{\sigma}_{1}^{0}=\sigma_{3}$. After calculating the traces, we obtain

$$
\sum_{\bar{s}_{e}, \bar{s}_{\mu}}\left(1 / 2, \bar{s}_{\mu}, 1 / 2, \bar{s}_{e} \mid F, m\right) \sum_{s_{e}, s_{\mu}}\left(1 / 2, s_{\mu}, 1 / 2, s_{e} \mid F, m\right)\left(\bar{u}_{\mu}^{\bar{s}_{\mu}} \gamma_{\alpha} P_{L} v_{e}^{\bar{s}_{e}}\right)\left(\bar{v}_{\mu}^{s_{\mu}} \gamma^{\alpha} P_{L} u_{e}^{s_{e}}\right)=2 m_{\mu} m_{e}
$$

\footnotetext{
${ }^{8}$ We note that the definition of the charge conjugations of the creation and annihilation operators can have unphysical sign freedom. We define the $\overline{\mathrm{Mu}}$ state by Eq. (A7).
} 
By the Fierz transformation, the third term is changed into

$$
\left(\bar{v}_{\mu}^{s_{\mu}} \gamma_{\alpha} P_{L} v_{e}^{\bar{s}_{e}}\right)\left(\bar{u}_{\mu}^{\bar{s}_{\mu}} \gamma^{\alpha} P_{L} u_{e}^{s_{e}}\right)=-\left(\bar{u}_{\mu}^{\bar{s}_{\mu}} \gamma_{\alpha} P_{L} v_{e}^{\bar{s}_{e}}\right)\left(\bar{v}_{\mu}^{s_{\mu}} \gamma^{\alpha} P_{L} u_{e}^{s_{e}}\right)
$$

The second and fourth terms are the same as the first and third ones in the case of the operator $Q_{1}$. Thus, we obtain

$$
\left\langle\overline{\mathrm{Mu}} ; F, m\left|Q_{1}\right| \mathrm{Mu} ; F, m\right\rangle=-8|\varphi(0)|^{2},
$$

for $(F, m)=(1, \pm 1),(1,0)$, and $(0,0)$.

By applying the similar procedure, we also find the amplitudes for $Q_{2}-Q_{5}$,

$$
\left\langle\overline{\mathrm{Mu}} ; F, m\left|Q_{2}\right| \mathrm{Mu} ; F, m\right\rangle=-8|\varphi(0)|^{2},
$$

$$
\left\langle\overline{\mathrm{Mu}} ; 0,0\left|Q_{3}\right| \mathrm{Mu} ; 0,0\right\rangle=12|\varphi(0)|^{2}, \quad\left\langle\overline{\mathrm{Mu}} ; 1, m\left|Q_{3}\right| \mathrm{Mu} ; 1, m\right\rangle=-4|\varphi(0)|^{2},
$$

$\left\langle\overline{\mathrm{Mu}} ; F, m\left|Q_{4}\right| \mathrm{Mu} ; F, m\right\rangle=\left\langle\overline{\mathrm{Mu}} ; F, m\left|Q_{5}\right| \mathrm{Mu} ; F, m\right\rangle=2|\varphi(0)|^{2}$.

We now easily obtain Eqs. (2.20) and (2.21). ${ }^{9}$

\section{APPENDIX B: MUONIUM GROUND-STATE SPECTROSCOPY}

The Zeeman effect splits the energy levels of the Mu states. The spin Hamiltonian of Mu is given as

$$
\mathcal{H}=a \boldsymbol{S}_{\mu} \cdot \boldsymbol{S}_{e}-\boldsymbol{\mu}_{e^{-}} \cdot \boldsymbol{B}-\boldsymbol{\mu}_{\mu^{+}} \cdot \boldsymbol{B}
$$

Here, $a=h \nu_{\mathrm{HFS}}=2 \pi \nu_{\mathrm{HFS}}$ is the HFS coupling constant, and $\boldsymbol{\mu}_{e^{-}}, \boldsymbol{\mu}_{\mu^{+}}$are the magnetic moments of the electron and antimuon:

$$
\boldsymbol{\mu}_{e^{-}}=-g_{e} \mu_{B} \boldsymbol{S}_{e}, \quad \boldsymbol{\mu}_{\mu^{+}}=g_{\mu} \frac{m_{e}}{m_{\mu}} \mu_{B} \boldsymbol{S}_{\mu},
$$

where $\mu_{B}$ is the Bohr magneton, and $g_{e, \mu}$ are $g$-factors of the electron and muon.

We first ignore the $\mathrm{Mu}-\overline{\mathrm{Mu}}$ mixing interactions. The energy eigenvalues of $\mathrm{Mu}$ states $|\mathrm{Mu} ; F, m\rangle_{B}$ are given as

$$
\begin{gathered}
E_{B}(\mathrm{Mu} ; 1, \pm 1)=E_{0}+\frac{a}{2}\left(\frac{1}{2} \pm Y\right), \\
E_{B}(\mathrm{Mu} ; 1,0)=E_{0}+\frac{a}{2}\left(-\frac{1}{2}+\sqrt{1+X^{2}}\right), \\
E_{B}(\mathrm{Mu} ; 0,0)=E_{0}+\frac{a}{2}\left(-\frac{1}{2}-\sqrt{1+X^{2}}\right) .
\end{gathered}
$$

Here, $E_{0} \simeq-m_{\text {red }} \alpha^{2} / 2$ is the $1 S$ binding energy and

$$
\begin{aligned}
& X=\frac{\mu_{B} B}{a}\left(g_{e}+\frac{m_{e}}{m_{\mu}} g_{\mu}\right) \simeq 6.31 \frac{B}{\text { Tesla }}, \\
& Y=\frac{\mu_{B} B}{a}\left(g_{e}-\frac{m_{e}}{m_{\mu}} g_{\mu}\right) \simeq 6.25 \frac{B}{\text { Tesla }},
\end{aligned}
$$

where we have used $\mu_{B} \simeq 5.788 \times 10^{-5} \mathrm{eV} /$ Tesla, $a \simeq 1.846 \times 10^{-5} \mathrm{eV}$, and $g_{e} \simeq g_{\mu} \simeq 2.002$. Since the response of $\overline{\mathrm{Mu}}$ to the magnetic field is opposite to that of $\mathrm{Mu}$, we obtain the formulas for $\overline{\mathrm{Mu}}$ by replacing $X$ and $Y$ with $-X$ and $-Y$, respectively. It is found that the energy values for $\overline{\mathrm{Mu}}$ are

\footnotetext{
${ }^{9}$ Reference [14] includes some typos in the signs of amplitudes.
} 


$$
\begin{gathered}
E_{B}(\overline{\mathrm{Mu}} ; 1, \pm 1)=E_{0}+\frac{a}{2}\left(\frac{1}{2} \mp Y\right), \\
E_{B}(\overline{\mathrm{Mu}} ; 1,0)=E_{0}+\frac{a}{2}\left(-\frac{1}{2}+\sqrt{1+X^{2}}\right), \\
E_{B}(\overline{\mathrm{Mu}} ; 0,0)=E_{0}+\frac{a}{2}\left(-\frac{1}{2}-\sqrt{1+X^{2}}\right) .
\end{gathered}
$$

We note that the $(0,0)$ and $(1,0)$ states for the zero magnetic field are mixed as

$$
\begin{aligned}
& \left(\begin{array}{l}
|\mathrm{Mu} ; 1,0\rangle_{B} \\
|\mathrm{Mu} ; 0,0\rangle_{B}
\end{array}\right)=\left(\begin{array}{cc}
C & -S \\
S & C
\end{array}\right)\left(\begin{array}{l}
|\mathrm{Mu} ; 1,0\rangle \\
|\mathrm{Mu} ; 0,0\rangle
\end{array}\right), \\
& \left(\begin{array}{l}
|\overline{\mathrm{Mu}} ; 1,0\rangle_{B} \\
|\overline{\mathrm{Mu}} ; 0,0\rangle_{B}
\end{array}\right)=\left(\begin{array}{cc}
C & S \\
-S & C
\end{array}\right)\left(\begin{array}{l}
|\overline{\mathrm{Mu}} ; 1,0\rangle \\
|\overline{\mathrm{Mu}} ; 0,0\rangle
\end{array}\right),
\end{aligned}
$$

where the mixing is given as

$$
C=\frac{1}{\sqrt{2}}\left(1+\frac{1}{\sqrt{1+X^{2}}}\right)^{\frac{1}{2}}, \quad S=\frac{1}{\sqrt{2}}\left(1-\frac{1}{\sqrt{1+X^{2}}}\right)^{\frac{1}{2}} .
$$

The experimental measurement of the HFS interval in the strong magnetic field is obtained as

$$
a=h \nu_{12}+h \nu_{34},
$$

where

$$
\begin{gathered}
h \nu_{12} \equiv E_{B}(\mathrm{Mu} ; 1,1)-E_{B}(\mathrm{Mu} ; 1,0)=\frac{a}{2}\left(1+Y-\sqrt{1+X^{2}}\right), \\
h \nu_{34} \equiv E_{B}(\mathrm{Mu} ; 1,-1)-E_{B}(\mathrm{Mu} ; 0,0)=\frac{a}{2}\left(1-Y+\sqrt{1+X^{2}}\right) .
\end{gathered}
$$

Now we switch on the $\mathrm{Mu}-\overline{\mathrm{Mu}}$ mixing interactions and $\mathcal{M}_{F, m} \neq 0$. To calculate the energy states, we drop the $\Gamma_{12}$ part of $\mathcal{M}_{12}=M_{12}-i \Gamma_{12} / 2$ and we denote the $M_{12}$ parts of $F=0$ and $F=1$ states as $M_{0}$ and $M_{1}$, which can be made to be real by rephasing of the states.

If the external magnetic field is zero, $\mathrm{Mu}$ and $\overline{\mathrm{Mu}}$ are maximally mixed and the energy eigenstates correspond to the $C P$ eigenstates:

$$
| \pm ; F, m\rangle \equiv \frac{1}{\sqrt{2}}(|\mathrm{Mu} ; F, m\rangle \pm|\overline{\mathrm{Mu}} ; F, m\rangle) .
$$

The energy matrix of $(|\mathrm{Mu}, 1,1\rangle,|\overline{\mathrm{Mu}}, 1,1\rangle)$ can be written as

$$
E_{0} \mathbf{1}+\left(\begin{array}{cc}
\frac{a}{4}+\frac{a}{2} Y & M_{1} \\
M_{1} & \frac{a}{4}-\frac{a}{2} Y
\end{array}\right)
$$

The energy eigenvalues are

$$
E_{(1,1), \pm}=E_{0}+\frac{a}{4} \pm \sqrt{\left(\frac{a}{2} Y\right)^{2}+M_{1}^{2}} \text {, }
$$

and $\Delta E \simeq a Y$. The $\mathrm{Mu}-\overline{\mathrm{Mu}}$ mixing becomes small in the magnetic field due to $\Delta E \gg M_{1}$. The $m=-1$ state is similar to the $m=1$ state. 
The energy matrix of $m=0$ states $(|\mathrm{Mu} ; 1,0\rangle,|\mathrm{Mu} ; 0,0\rangle,|\overline{\mathrm{Mu}} ; 1,0\rangle,|\overline{\mathrm{Mu}} ; 0,0\rangle)$ in an appropriate sign convention can be written as

$$
E_{0} \mathbf{1}+\left(\begin{array}{cccc}
\frac{a}{4} & -\frac{a}{2} X & M_{1} & 0 \\
-\frac{a}{2} X & -\frac{3}{4} a & 0 & M_{0} \\
M_{1} & 0 & \frac{a}{4} & \frac{a}{2} X \\
0 & M_{0} & \frac{a}{2} X & -\frac{3}{4} a
\end{array}\right)
$$

The energy eigenstates in the magnetic field are

$$
\begin{aligned}
& |+; 1,0\rangle \cos \theta_{M}-|-; 0,0\rangle \sin \theta_{M} \simeq\left(|\mathrm{Mu} ; 1,0\rangle_{B}+|\overline{\mathrm{Mu}} ; 1,0\rangle_{B}\right) / \sqrt{2}, \\
& |+; 1,0\rangle \sin \theta_{M}+|-; 0,0\rangle \cos \theta_{M} \simeq\left(|\mathrm{Mu} ; 0,0\rangle_{B}-|\overline{\mathrm{Mu}} ; 0,0\rangle_{B}\right) / \sqrt{2}, \\
& |-; 1,0\rangle \cos \theta_{\bar{M}}-|+; 0,0\rangle \sin \theta_{\bar{M}} \simeq\left(|\mathrm{Mu} ; 1,0\rangle_{B}-|\overline{\mathrm{Mu}} ; 1,0\rangle_{B}\right) / \sqrt{2}, \\
& |-; 1,0\rangle \sin \theta_{\bar{M}}+|+; 0,0\rangle \cos \theta_{\bar{M}} \simeq\left(|\mathrm{Mu} ; 0,0\rangle_{B}+|\overline{\mathrm{Mu}} ; 0,0\rangle_{B}\right) / \sqrt{2},
\end{aligned}
$$

where

$$
\tan 2 \theta_{M}=\frac{a X}{a+M_{1}+M_{0}}, \quad \tan 2 \theta_{\bar{M}}=\frac{a X}{a-M_{1}-M_{0}}
$$

The energy eigenvalues are

$$
\begin{aligned}
E_{(1,0), \pm} & =E_{0}-\frac{a}{4} \pm \frac{M_{1}-M_{0}}{2}+\frac{1}{2} \sqrt{\left(a \pm M_{1} \pm M_{0}\right)^{2}+(a X)^{2}} \\
& \simeq E_{0}-\frac{a}{4}+\frac{a}{2} \sqrt{1+X^{2}} \pm\left(\frac{M_{1}-M_{0}}{2}+\frac{M_{1}+M_{0}}{2 \sqrt{1+X^{2}}}\right), \\
E_{(0,0), \pm} & =E_{0}-\frac{a}{4} \pm \frac{M_{1}-M_{0}}{2}-\frac{1}{2} \sqrt{\left(a \pm M_{1} \pm M_{0}\right)^{2}+(a X)^{2}} \\
& \simeq E_{0}-\frac{a}{4}-\frac{a}{2} \sqrt{1+X^{2}} \mp\left(-\frac{M_{1}-M_{0}}{2}+\frac{M_{1}+M_{0}}{2 \sqrt{1+X^{2}}}\right) .
\end{aligned}
$$

The precise development is described by the $4 \times 4$ matrix, but the transitions can approximately happen only $|\mathrm{Mu} ; 1,0\rangle_{B} \rightarrow$ $|\overline{\mathrm{Mu}} ; 1,0\rangle_{B}$ and $|\mathrm{Mu} ; 0,0\rangle_{B} \rightarrow|\overline{\mathrm{Mu}} ; 0,0\rangle_{B}$, and the mass differences are

$$
\begin{gathered}
\frac{\Delta M_{1,0}^{B}}{2}\left(=M_{1}^{B}\right)=C^{2} M_{1}-S^{2} M_{0}=\frac{M_{1}-M_{0}}{2}+\frac{M_{1}+M_{0}}{2 \sqrt{1+X^{2}}}, \\
\frac{\Delta M_{0,0}^{B}}{2}\left(=M_{0}^{B}\right)=C^{2} M_{0}-S^{2} M_{1}=-\frac{M_{1}-M_{0}}{2}+\frac{M_{1}+M_{0}}{2 \sqrt{1+X^{2}}} .
\end{gathered}
$$

\section{APPENDIX C: DIAGONALIZATION OF NEUTRINO MASS MATRIX}

We work on the basis where the charged-lepton mass matrix is diagonal. The $6 \times 6$ neutrino mass matrix $\mathcal{M}$ is written as

$$
-\mathcal{L}_{m}=\frac{1}{2}\left(\overline{\left(\nu^{c}\right)_{R}} \quad \overline{N_{R}}\right) \mathcal{M}\left(\begin{array}{c}
\nu_{L} \\
\left(N^{c}\right)_{L}
\end{array}\right)+\text { H.c. }
$$

where $\nu$ and $N$ are current-basis left- and right-handed neutrinos, and 


$$
\mathcal{M}=\left(\begin{array}{cc}
0 & m_{D} \\
m_{D}^{T} & M_{N}
\end{array}\right)
$$

The mass eigenstates $\nu^{\prime}, N^{\prime}$ are given as

$$
\left(\begin{array}{c}
\nu_{L} \\
\left(N^{c}\right)_{L}
\end{array}\right)=\mathcal{U}\left(\begin{array}{c}
\nu_{L}^{\prime} \\
N_{L}^{\prime}
\end{array}\right)
$$

and

$$
\mathcal{U}^{T} \mathcal{M U}=\operatorname{diag}\left(M_{\mathcal{I}}\right)=\operatorname{diag}\left(m_{i}, M_{I}\right) .
$$

We choose phases in $\mathcal{U}$ so that $M_{\mathcal{I}}$ 's are real. We use index $i$ for the light neutrino mass eigenstates, index $I$ for the "heavy" neutrino mass eigenstates, and index $\mathcal{I}$ for both states. We use the Greek characters $\alpha, \beta$ for the generation index in the current basis. For convenience, we define

$$
\mathcal{U}=\left(\begin{array}{ll}
U & X \\
V & Y
\end{array}\right)
$$

Namely,

$$
\begin{aligned}
& \left(\nu_{L}\right)_{\alpha}=U_{\alpha i} \nu_{i}^{\prime}+X_{\alpha I} N_{I}^{\prime}, \\
& \left(N_{L}^{c}\right)_{\alpha}=V_{\alpha i} \nu_{i}^{\prime}+Y_{\alpha I} N_{I}^{\prime} .
\end{aligned}
$$

In the following, the mass eigenstates $\nu_{i}$ and $N_{I}$ are defined as Majorana fermions, e.g., $\nu_{i}=\nu_{i}^{\prime}+\left(\nu_{i}^{\prime}\right)^{c}$.

The interactions to the $W_{L}$ and $W_{R}$ gauge bosons are written as

$$
\begin{aligned}
& \frac{g_{L}}{\sqrt{2}} W_{L}^{\mu} \bar{l}_{L}^{\alpha} \gamma_{\mu}\left(U_{\alpha i} \nu_{i}+X_{\alpha I} N_{I}\right)_{L} \\
& \quad+\frac{g_{R}}{\sqrt{2}} W_{R}^{\mu} \bar{l}_{R}^{\alpha} \gamma_{\mu}\left(V_{\alpha i}^{*} \nu_{i}+Y_{\alpha I}^{*} N_{I}\right)_{R}+\text { H.c. }
\end{aligned}
$$

If one adds three gauge singlets $S$, the expressions can be easily extended. We define so that the $9 \times 9$ mass matrix $\mathcal{M}$ for $\mathcal{N}=\left(\nu_{L},\left(N^{c}\right)_{L}, S\right)^{T}$ is diagonalized by $9 \times 9$ diagonalization unitary matrix

$$
\mathcal{U}=\left(\begin{array}{cc}
U & X \\
V & Y \\
W & Z
\end{array}\right),
$$

where $U, V, W$ are $3 \times 3$ matrices, and $X, Y, Z$ are $3 \times 6$ matrices. Then, the expression of the gauge interaction is unchanged under this convention, but the indices of the "heavy" neutrinos are summed by $I=1,2, \ldots, 6$.

\section{APPENDIX D: BOX LOOP FUNCTION}

In the box loop calculation, one encounters an integral such as

$$
I_{n}(x, y, z ; \xi)=\int_{0}^{\infty} d t \frac{(-t)^{n}}{(t+x)(t+y)(t+z)(t+1)\left(t+\xi_{1}\right)^{a_{1}}\left(t+\xi_{2} z\right)^{a_{2}}},
$$

where $a_{i}=0$ or 1 , and $\xi_{i}$ is a gauge parameter for $R_{\xi}$ gauge, e.g., $\xi_{1}=\xi_{2}=\xi$ and $z=1$ in the box diagram with $W_{L}-W_{L}$ exchanges. Since

$$
\frac{t^{2}}{(t+x)(t+y)}=1-\frac{x}{t+x}-\frac{y}{t+y}+\frac{x y}{(t+x)(t+y)},
$$

one finds

$$
\sum_{i, j} \lambda_{i} \lambda_{j} I_{n+2}\left(x_{i}, x_{j}, z ; \xi\right)=\sum_{i, j} \lambda_{i} \lambda_{j} x_{i} x_{j} I_{n}\left(x_{i}, x_{j}, z ; \xi\right)
$$

if $\sum_{i} \lambda_{i}=0$. Due to this equation, the $\xi$ dependence in the box loop calculation can vanish. For example, for

$$
\lambda_{i}=V_{\alpha i} V_{\beta i}^{*}, \quad x_{i}=\frac{m_{i}^{2}}{M_{W}^{2}},
$$

$\sum_{i} \lambda_{i}=0$ is satisfied due the unitarity of the mixing matrix $V$, and thus, the box loop contribution of the meson mixings is gauge independent [83]. 
We define

$$
\begin{aligned}
I_{n}(x, y, z) & =\int_{0}^{\infty} d t \frac{(-t)^{n}}{(t+x)(t+y)(t+z)(t+1)} \\
& =\frac{x^{n} \ln x}{(x-1)(x-y)(x-z)}+\frac{y^{n} \ln y}{(y-1)(y-x)(y-z)}+\frac{z^{n} \ln z}{(z-1)(z-x)(z-y)},
\end{aligned}
$$

for $n=0,1,2$, and the loop functions of the box diagrams are given as

$$
\begin{gathered}
E_{0}(x, y, z)=x y\left(I_{0}(x, y, z)-\left(1+\frac{1}{z}\right) I_{1}(x, y, z)+\frac{1}{4 z} I_{2}(x, y, z)\right), \\
E_{1}(x, y, z)=2 \sqrt{x y}\left(I_{1}(x, y, z)+\frac{x y}{4 z} I_{1}(x, y, z)-\frac{1}{4}\left(1+\frac{1}{z}\right) I_{2}(x, y, z)\right),
\end{gathered}
$$

where $E_{0}$ is a function for the contributions where momentums $(\not k)$ are picked in the fermion propagators, and $E_{1}$ is a function for the ones where masses $\left(M_{\mathcal{I}}\right)$ are picked. The coefficients of the transition operators can be written by $E_{0}$ and $E_{1}$ terms. The gauge invariance of the $E_{0}$ term is assured by the unitarity of the neutrino mixing matrix $\mathcal{U}$. On the other hand, $E_{1}$ term is not necessarily gauge invariant. For the $W_{L}-W_{L}$ exchange diagram in the type-I seesaw case, the function $E_{1}$ is gauge invariant due to

$$
\mathcal{U}^{*} \mathcal{M}^{\text {diag }} \mathcal{U}^{\dagger}=\mathcal{M}
$$

and

$$
\left(\mathcal{U}^{*} \mathcal{M}^{\text {diag }} \mathcal{U}^{\dagger}\right)_{e_{L}, e_{L}}=0, \quad\left(\mathcal{U}^{*} \mathcal{M}^{\text {diag }} \mathcal{U}^{\dagger}\right)_{\mu_{L}, \mu_{L}}=0 .
$$

Similarly, for the $W_{R}-W_{R}$ exchange diagram in the leftright model without $S U(2)_{R}$ triplet, the $E_{1}$ term is gauge invariant. However, for the $W_{R}-W_{R}$ exchange with $S U(2)_{R}$ triplet, and $W_{L}-W_{R}$ exchange box diagrams, the $E_{1}$ term is not gauge invariant. In those cases, adding the loop corrections of the triplet and bidoublet Higgs scalar exchange diagram, the gauge dependence is canceled.
See Refs. [84,85] for the gauge invariance in the case of the $K-\bar{K}$ mixing. (Strictly speaking, in the case of the type-II seesaw with $S U(2)_{L}$ triplet, the box loop is not gauge invariant similarly unless the light neutrino masses are ignored.) The loop function $E_{1}$ above is given in the 't Hooft-Feynman gauge.

The loop function $E_{0}$ is usually redefined as given by the Inami-Lim function [83],

$$
\begin{aligned}
\tilde{E}_{0}(x, y, z)= & E_{0}(x, y, z)-E_{0}(x, 0, z)-E_{0}(0, y, z) \\
& +E_{0}(0,0, z) .
\end{aligned}
$$

The function $E_{0}$ above is already redefined using Eq. (D3) by the unitarity of the mixing matrix, and thus, $\tilde{E}_{0}(x, y, z)=E_{0}(x, y, z)$.

To express the loop functions for $W_{L}-W_{L}$ and $W_{R}-W_{R}$ box diagrams shortly, we define

$$
E_{0}(x, y) \equiv E_{0}(x, y, 1), \quad E_{1}(x, y) \equiv E_{1}(x, y, 1) .
$$

We note

$$
\begin{aligned}
I_{n}(x, y) & \equiv I_{n}(x, y, 1) \\
& =\frac{x^{n} \ln x}{(1-x)^{2}(x-y)}+\frac{y^{n} \ln y}{(1-y)^{2}(y-x)}+\frac{1}{(1-x)(1-y)} \\
& =\frac{1}{x-y}\left(\frac{x^{n} \ln x}{(1-x)^{2}}-\frac{y^{n} \ln y}{(1-y)^{2}}+\frac{1}{1-x}-\frac{1}{1-y}\right),
\end{aligned}
$$

and

$$
\begin{gathered}
I_{n}(x, x, z)=\frac{z^{n} \ln z}{(z-1)(x-z)^{2}}+\frac{x^{n-1}(1+n \ln x)}{(x-1)(x-z)}-\frac{(2 x-1-z) x^{n} \ln x}{(x-1)^{2}(x-z)^{2}}, \\
I_{n}(x, x)=\frac{1+x^{n-1}(1+n \ln x)}{(1-x)^{2}}+\frac{2 x^{n} \ln x}{(1-x)^{3}} .
\end{gathered}
$$


[1] B. Pontecorvo, Mesonium and anti-mesonium, Sov. Phys. JETP 6, 429 (1957).

[2] G. Feinberg and S. Weinberg, Conversion of muonium into antimuonium, Phys. Rev. 123, 1439 (1961).

[3] B.W. Lee and R. E. Shrock, Natural suppression of symmetry violation in gauge theories: Muon-lepton and electron lepton number nonconservation, Phys. Rev. D 16, 1444 (1977); B. W. Lee, S. Pakvasa, R. E. Shrock, and H. Sugawara, Muon and Electron Number Nonconservation in a V-A Gauge Model, Phys. Rev. Lett. 38, 937 (1977); 38, 1230(E) (1977).

[4] A. Halprin, Neutrinoless Double Beta Decay and Muonium-Anti-Muonium Transitions, Phys. Rev. Lett. 48, 1313 (1982).

[5] L. Willmann, P. V. Schmidt, H. P. Wirtz, R. Abela, V. Baranov, J. Bagaturia, W. H. Bertl, R. Engfer, A. Grossmann, V. W. Hughes et al., New Bounds from Searching for Muonium to Anti-Muonium Conversion, Phys. Rev. Lett. 82, 49 (1999).

[6] N. Kawamura, R. Kitamura, H. Yasuda, M. Otani, Y. Nakazawa, H. Iinuma, and T. Mibe, A new approach for $\mathrm{Mu}-\overline{\mathrm{Mu}}$ conversion search, J. Phys. Soc. Jpn. Conf. Proc. 33, 011120 (2021).

[7] J. Tang et al. (MACE Working Group Collaboration), Search for Muonium to Antimuonium Conversion, Letter of interest contribution to snowmass21, https:/www.snowmass21.org/ docs/files/summaries/RF/SNOWMASS21-RF5_RF0_Jian_ Tang-126.pdf.

[8] J. Adam et al. (MEG Collabortion), New Constraint on the Existence of the $\mu^{+} \rightarrow e^{+} \gamma$ Decay, Phys. Rev. Lett. 110, 201801 (2013); A. M. Baldini et al. (MEG Collaboration), Search for the lepton flavour violating decay $\mu^{+} \rightarrow \mathrm{e}^{+} \gamma$ with the full dataset of the MEG experiment, Eur. Phys. J. C 76, 434 (2016).

[9] U. Bellgardt et al. (SINDRUM Collaboration), Search for the decay $\mu^{+} \rightarrow e^{+} e^{+} e^{-}$, Nucl. Phys. B299, 1 (1988).

[10] A. Zee, A theory of lepton number violation, neutrino Majorana mass, and oscillation, Phys. Lett. 93B, 389 (1980); 95B, 461(E) (1980).

[11] A. Zee, Quantum numbers of Majorana neutrino masses, Nucl. Phys. B264, 99 (1986).

[12] K. S. Babu, Model of 'calculable' Majorana neutrino masses, Phys. Lett. B 203, 132 (1988).

[13] K. S. Babu and C. Macesanu, Two loop neutrino mass generation and its experimental consequences, Phys. Rev. D 67, 073010 (2003).

[14] R. Conlin and A. A. Petrov, Muonium-antimuonium oscillations in effective field theory, Phys. Rev. D 102, 095001 (2020).

[15] K. Horikawa and K. Sasaki, Muonium-anti-muonium conversion in models with dilepton gauge bosons, Phys. Rev. D 53, 560 (1996).

[16] W. S. Hou and G. G. Wong, Magnetic field dependence of muonium-anti-muonium conversion, Phys. Lett. B 357, 145 (1995).

[17] S. Nishimura et al. (MuSEUM Collaboration), Rabioscillation spectroscopy of the hyperfine structure of muonium atoms, Phys. Rev. A 104, L020801 (2021).

[18] K. S. Tanaka, M. Iwasaki, O. Kamigaito, S. Kanda, N. Kawamura, Y. Matsuda, T. Mibe, S. Nishimura, N. Saito and N. Sakamoto et al. Development of microwave cavities for measurement of muonium hyperfine structure at J-PARC, arXiv:2104.06663.

[19] W. Liu, M. G. Boshier, S. Dhawan, O. van Dyck, P. Egan, X. Fei, M. Grosse Perdekamp, V. Hughes, M. Janousch, K. Jungmann et al., High Precision Measurements of the Ground State Hyperfine Structure Interval of Muonium and of the Muon Magnetic Moment, Phys. Rev. Lett. 82, 711 (1999).

[20] P. J. Mohr, D. B. Newell, and B. N. Taylor, CODATA recommended values of the fundamental physical constants: 2014, Rev. Mod. Phys. 88, 035009 (2016).

[21] M. I. Eides, Hyperfine splitting in muonium: Accuracy of the theoretical prediction, Phys. Lett. B 795, 113 (2019).

[22] S. G. Karshenboim and E. Y. Korzinin, Theoretical prediction for the muonium hyperfine-structure interval and its accuracy, Phys. Rev. A 103, 022805 (2021).

[23] H. Fujii, Y. Mimura, K. Sasaki, and T. Sasaki, Muonium hyperfine structure and the decay $\mu^{+} \rightarrow e^{+}+\bar{\nu}_{e}+\nu_{\mu}$ in models with dilepton gauge baryons, Phys. Rev. D 49, 559 (1994).

[24] T. E. Clark and S. T. Love, Muonium-antimuonium oscillations and massive Majorana neutrinos, Mod. Phys. Lett. A 19, 297 (2004).

[25] G. Cvetic, C. O. Dib, C. S. Kim, and J. D. Kim, Muoniumantimuonium conversion in models with heavy neutrinos, Phys. Rev. D 71, 113013 (2005).

[26] B. Liu, Gauge invariance of the muonium-antimuonium oscillation time scale and limits on right-handed neutrino masses, Mod. Phys. Lett. A 24, 335 (2009).

[27] A. Abada, V. De Romeri, and A. M. Teixeira, Impact of sterile neutrinos on nuclear-assisted cLFV processes, J. High Energy Phys. 02 (2016) 083; A. Abada, J. Kriewald, and A. M. Teixeira, On the role of leptonic CPV phases in cLFV observables, arXiv:2107.06313.

[28] A. Atre, T. Han, S. Pascoli, and B. Zhang, The search for heavy Majorana neutrinos, J. High Energy Phys. 05 (2009) 030.

[29] F. F. Deppisch, P. S. Bhupal Dev, and A. Pilaftsis, Neutrinos and collider physics, New J. Phys. 17, 075019 (2015).

[30] A. Pilaftsis, Radiatively induced neutrino masses and large Higgs neutrino couplings in the standard model with Majorana fields, Z. Phys. C 55, 275 (1992).

[31] D. Chang and W. Y. Keung, Constraints on MuoniumantiMuonium Conversion, Phys. Rev. Lett. 62, 2583 (1989).

[32] M. L. Swartz, Limits on doubly charged Higgs bosons and lepton flavor violation, Phys. Rev. D 40, 1521 (1989).

[33] J. Schechter and J. W.F. Valle, Neutrino masses in $\mathrm{SU}(2) \times \mathrm{U}(1)$ theories, Phys. Rev. D 22, 2227 (1980).

[34] T. P. Cheng and L. F. Li, Neutrino masses, mixings and oscillations in $\mathrm{SU}(2) \times \mathrm{U}(1)$ models of electroweak interactions, Phys. Rev. D 22, 2860 (1980).

[35] G. Lazarides, Q. Shafi, and C. Wetterich, Proton lifetime and Fermion masses in an SO(10) model, Nucl. Phys. B181, 287 (1981).

[36] R. N. Mohapatra and G. Senjanovic, Neutrino masses and mixings in gauge models with spontaneous parity violation, Phys. Rev. D 23, 165 (1981).

[37] C. Han, D. Huang, J. Tang, and Y. Zhang, Probing the doubly-charged Higgs with muonium to antimuonium conversion experiment, Phys. Rev. D 103, 055023 (2021). 
[38] P. A. Zyla et al. (Particle Data Group), Review of particle physics, Prog. Theor. Exp. Phys. 2020, 083C01 (2020).

[39] M. Raidal and A. Santamaria, Muon electron conversion in nuclei versus mu $\rightarrow$ e gamma: An Effective field theory point of view, Phys. Lett. B 421, 250 (1998).

[40] N. Aghanim et al. (Planck Collaboration), Planck 2018 results. VI. Cosmological parameters, Astron. Astrophys. 641, A6 (2020).

[41] Z. Chacko, A. Dev, P. Du, V. Poulin, and Y. Tsai, Cosmological limits on the neutrino mass and lifetime, J. High Energy Phys. 04 (2020) 020.

[42] M. Aaboud et al. (ATLAS Collaboration), Search for doubly charged Higgs boson production in multi-lepton final states with the ATLAS detector using proton-proton collisions at $\sqrt{s}=13$ TeV, Eur. Phys. J. C 78, 199 (2018).

[43] P. S. B. Dev, M. J. Ramsey-Musolf, and Y. Zhang, Doublycharged scalars in the type-II seesaw mechanism: Fundamental symmetry tests and high-energy searches, Phys. Rev. D 98, 055013 (2018).

[44] P. B. Pal, Constraints on a muon-neutrino mass around 100-keV, Nucl. Phys. B227, 237 (1983).

[45] P. Herczeg and R. N. Mohapatra, Muonium to AntiMuonium Conversion and the Decay $\mu^{+} \rightarrow e^{+}$AntiNeutrino Neutrino in Left-Right Symmetric Models, Phys. Rev. Lett. 69, 2475 (1992).

[46] M. Blanke, A. J. Buras, K. Gemmler, and T. Heidsieck, Delta $\mathrm{F}=2$ observables and $B \rightarrow X_{q} \gamma$ decays in the leftright Model: Higgs particles striking back, J. High Energy Phys. 03 (2012) 024.

[47] S. Bertolini, A. Maiezza, and F. Nesti, Present and future K and $\mathrm{B}$ meson mixing constraints on $\mathrm{TeV}$ scale left-right symmetry, Phys. Rev. D 89, 095028 (2014).

[48] M. Nemevšek, F. Nesti, and G. Popara, Keung-Senjanović process at the LHC: From lepton number violation to displaced vertices to invisible decays, Phys. Rev. D 97, 115018 (2018).

[49] A. M. Sirunyan et al. (CMS Collaboration), Search for a heavy right-handed $\mathrm{W}$ boson and a heavy neutrino in events with two same-flavor leptons and two jets at $\sqrt{s}=13 \mathrm{TeV}$, J. High Energy Phys. 05 (2018) 148.

[50] M. Aaboud et al. (ATLAS Collaboration), Search for a right-handed gauge boson decaying into a high-momentum heavy neutrino and a charged lepton in $p p$ collisions with the ATLAS detector at $\sqrt{s}=13 \mathrm{TeV}$, Phys. Lett. B 798, 134942 (2019).

[51] J. Barry and W. Rodejohann, Lepton number and flavour violation in $\mathrm{TeV}$-scale left-right symmetric theories with large left-right mixing, J. High Energy Phys. 09 (2013) 153.

[52] P. S. Bhupal Dev, S. Goswami, and M. Mitra, TeV scale leftright symmetry and large mixing effects in neutrinoless double beta decay, Phys. Rev. D 91, 113004 (2015).

[53] A. Das, P. S. B. Dev, and R. N. Mohapatra, Same sign versus opposite sign dileptons as a probe of low scale seesaw mechanisms, Phys. Rev. D 97, 015018 (2018).

[54] K. L. McDonald and B. H. J. McKellar, Evaluating the two loop diagram responsible for neutrino mass in Babu's model, arXiv:hep-ph/0309270.

[55] M. Nebot, J. F. Oliver, D. Palao, and A. Santamaria, Prospects for the Zee-Babu model at the CERN LHC and low energy experiments, Phys. Rev. D 77, 093013 (2008).
[56] J. Herrero-Garcia, M. Nebot, N. Rius, and A. Santamaria, The Zee-Babu model revisited in the light of new data, Nucl. Phys. B885, 542 (2014).

[57] M. Gustafsson, J. M. No, and M. A. Rivera, Predictive Model for Radiatively Induced Neutrino Masses and Mixings with Dark Matter, Phys. Rev. Lett. 110, 211802 (2013); 112, 259902(E) (2014).

[58] R. Cepedello, M. Hirsch, P. Rocha-Morán, and A. Vicente, Minimal 3-loop neutrino mass models and charged lepton flavor violation, J. High Energy Phys. 08 (2020) 067.

[59] E. Ma, Verifiable radiative seesaw mechanism of neutrino mass and dark matter, Phys. Rev. D 73, 077301 (2006).

[60] L. M. Krauss, S. Nasri, and M. Trodden, A Model for neutrino masses and dark matter, Phys. Rev. D 67, 085002 (2003).

[61] M. Aoki, S. Kanemura, and O. Seto, Neutrino Mass, Dark Matter and Baryon Asymmetry via TeV-Scale Physics without Fine-Tuning, Phys. Rev. Lett. 102, 051805 (2009).

[62] T. Fukuyama, K. Ichikawa, and Y. Mimura, Relation between proton decay and PMNS phase in the minimal SUSY SO(10) GUT, Phys. Lett. B 764, 114 (2017).

[63] I. Esteban, M. C. Gonzalez-Garcia, M. Maltoni, T. Schwetz, and A. Zhou, The fate of hints: updated global analysis of three-flavor neutrino oscillations, J. High Energy Phys. 09 (2020) 178.

[64] W. S. Hou and G. G. Wong, $\mu^{+} e^{-} \leftrightarrow \mu^{-} e^{+}$transitions via neutral scalar bosons, Phys. Rev. D 53, 1537 (1996).

[65] A. Halprin and A. Masiero, Muonium-antimuonium oscillations and exotic muon decay in broken R-parity SUSY models, Phys. Rev. D 48, R2987 (1993).

[66] F. Gabbiani, E. Gabrielli, A. Masiero, and L. Silvestrini, A complete analysis of FCNC and $C P$ constraints in general SUSY extensions of the standard model, Nucl. Phys. B477, 321 (1996).

[67] J. Hisano, T. Moroi, K. Tobe, M. Yamaguchi, and T. Yanagida, Lepton flavor violation in the supersymmetric standard model with seesaw induced neutrino masses, Phys. Lett. B 357, 579 (1995); J. Hisano, T. Moroi, K. Tobe, and M. Yamaguchi, Lepton flavor violation via right-handed neutrino Yukawa couplings in supersymmetric standard model, Phys. Rev. D 53, 2442 (1996).

[68] P. Candia da Silva and A. Pilaftsis, Radiative neutrino masses in the $\nu_{R}$ MSSM, Phys. Rev. D 102, 095013 (2020).

[69] P. H. Frampton and B. H. Lee, SU(15) Grand Unification, Phys. Rev. Lett. 64, 619 (1990).

[70] P. H. Frampton, Chiral Dilepton Model and the Flavor Question, Phys. Rev. Lett. 69, 2889 (1992).

[71] P. H. Frampton and D. Ng, Dileptons: Present status and future prospects, Phys. Rev. D 45, 4240 (1992).

[72] P. H. Frampton and M. Harada, Constraints from precision electroweak data on leptoquarks and bileptons, Phys. Rev. D 58, 095013 (1998).

[73] H. Fujii, S. Nakamua, and K. Sasaki, Constraints on dilepton mass from low-energy muon experiments, Phys. Lett. B 299, 342 (1993).

[74] Y. Koide, New U(3) family gauge symmetry and muonium into antimuonium conversion, arXiv:1009.4254.

[75] B. Abi et al. (Muon g-2 Collaboration), Measurement of the Positive Muon Anomalous Magnetic Moment to 0.46 ppm, Phys. Rev. Lett. 126, 141801 (2021). 
[76] R. H. Parker, C. Yu, W. Zhong, B. Estey, and H. Müller, Measurement of the fine-structure constant as a test of the standard model, Science 360, 191 (2018).

[77] L. Morel, Z. Yao, P. Cladé, and S. Guellati-Khélifa, Determination of the fine-structure constant with an accuracy of 81 parts per trillion, Nature (London) 588, 61 (2020).

[78] R. Aaij et al. (LHCb Collaboration), Test of lepton universality in beauty-quark decays, arXiv:2103.11769.

[79] M. Bauer and M. Neubert, Minimal Leptoquark Explanation for the $\mathrm{R}_{D^{(*)}}, \mathrm{R}_{K}$, and $(g-2)_{g}$ Anomalies, Phys. Rev. Lett. 116, 141802 (2016).

[80] L. Calibbi, D. Redigolo, R. Ziegler, and J. Zupan, Looking forward to Lepton-flavor-violating ALPs, J. High Energy Phys. 09 (2021) 173.

[81] M. Bauer, M. Neubert, S. Renner, M. Schnubel, and A. Thamm, Axionlike Particles, Lepton-Flavor Violation, and a
New Explanation of $a_{\mu}$ and $a_{e}$, Phys. Rev. Lett. 124, 211803 (2020).

[82] M. Endo, S. Iguro, and T. Kitahara, Probing $e \mu$ flavorviolating ALP at Belle II, J. High Energy Phys. 06 (2020) 040.

[83] T. Inami and C. S. Lim, Effects of superheavy quarks and leptons in low-energy weak processes $K_{L} \rightarrow \mu \bar{\nu}_{\mu}, K^{+} \rightarrow$ $\pi^{+} \nu \bar{\nu}$ and $K^{0} \leftrightarrow \bar{K}^{0}$, Prog. Theor. Phys. 65, 297 (1981); 65, 1772(E) (1981).

[84] W. S. Hou and A. Soni, Gauge invariance of the $K_{L}-K_{S}$ mass difference in left-right symmetric model, Phys. Rev. D 32, 163 (1985).

[85] J. Basecq, L. F. Li, and P. B. Pal, Gauge invariant calculation of the $K_{L}-K_{S}$ mass difference in the left-right model, Phys. Rev. D 32, 175 (1985). 